\title{
Asynchronous CSMA Policies in Multihop Wireless Networks with Primary Interference Constraints
}

\author{
Peter Marbach, Atilla Eryilmaz, and Asu Ozdaglar
}

\begin{abstract}
We analyze Asynchronous Carrier Sense Multiple Access (CSMA) policies for scheduling packet transmissions in multihop wireless networks subject to collisions under primary interference constraints. While the (asymptotic) achievable rate region of CSMA policies for single-hop networks has been wellknown, their analysis for general multihop networks has been an open problem due to the complexity of complex interactions among coupled interference constraints. Our work resolves this problem for networks with primary interference constraints by introducing a novel fixed-point formulation that approximates the link service rates of CSMA policies.

This formulation allows us to derive an explicit characterization of the achievable rate region of CSMA policies for a limiting regime of large networks with a small sensing period. Our analysis also reveals the rate at which CSMA achievable rate region approaches the asymptotic capacity region of such networks. Moreover, our approach enables the computation of approximate CSMA link transmission attempt probabilities to support any given arrival vector within the achievable rate region. As part of our analysis, we show that both of these approximations become (asymptotically) accurate for large networks with a small sensing period. Our numerical case studies further suggest that these approximations are accurate even for moderately sized networks.
\end{abstract}

Index Terms-Asymptotic Capacity Region of Wireless Networks, Carrier-Sense Multiple Access, Fixed-Point Approximation, Throughput-Optimal Scheduling.

\section{INTRODUCTION}

The design of efficient resource allocation algorithms for wireless networks has been an active area of research for decades. The seminal work [38] of Tassiulas and Ephremides has pioneered in a new thread of resource allocation mechanisms that are throughput-optimal in the sense that the algorithm stabilizes the network queues for flow rates that are stabilizable by any other algorithm. This and subsequent works (e.g. [36], [1], [10], [34], [32], [26], [11]) have proposed schemes that use queue-lengths to dynamically perform variety of resource allocation decisions, including medium access, routing, power control, and scheduling.

Scheduling (or medium access) has traditionally been the most computationally heavy and complex component of resource allocation strategies due to the interference-limited

P. Marbach is with the Department of Computer Science at the University of Toronto, Toronto, Canada. Email: marbachecs.toronto. edu.

A. Eryilmaz is with the Department of Electrical and Computer Engineering at the Ohio State University, Columbus, USA. Email: eryilmazdece. osu.edu.

A. Ozdaglar is with the Department of Electrical Engineering and Computer Science at the Massachusetts Institute of Technology, Cambridge, USA. Email: asumanemit. edu.

This work was supported in part by DTRA Grant HDTRA 1-08-1-0016, and NSF Awards: CAREER-CNS-0953515 and CCF-0916664. nature of the wireless medium. The queue-length-based policies typically have scheduling rules that use the queue-length information to avoid collisions while prioritizing the service of more heavily loaded nodes. However, due to the coupling between the interference constraints of nearby transmissions, such scheduling decisions can require highly complex and centralized decisions. This observation has motivated high research activity in the recent years for the development of distributed and low-complexity implementations of queuelength-based schemes (e.g. [37], [13], [7], [25], [8], [30], [41], [9], [42], [19]). Also, random access strategies have been investigated in a number of works (e.g. [22], [24], [39], [6], [16], [14], [35]) that achieve a fraction of the capacity region. In the case of primary interference model and general network topology that we consider, this fraction is $1 / 2$ and is tight (i.e. there exist networks for which no rate outside half of the capacity region can be supported). These results have suggested that a significant portion of the capacity region may need to be sacrificed to achieve distributed implementation with random access strategies. Besides performance degradation, the practical implementation of existing resource allocation policies are also complicated by several factors: they usually rely on global synchronization of transmissions and require a fair amount of information sharing (typically in the form of queue-lengths) between nodes to perform decisions.

In this work, we consider an alternative class of random access strategies with favorable complexity and practical implementability characteristics. In particular, we investigate Carrier Sense Multiple Access (CSMA) policies in which nodes operate asynchronously and sense the wireless channel before making an attempt to transmit a packet, which may result in collisions. We analyze such asynchronous CSMA policies for scheduling packet transmissions in multihop wireless networks subject to collisions under primary interference constraints. For a limiting regime of large networks with a small sensing period, we derive an explicit characterization of the achievable rate region of CSMA policies. While an explicit characterization of the (asymptotic) achievable rate region of CSMA policies has been established in the special case of singlehop networks, their analysis for general multihop networks has been an open problem due to the complexity of the interactions among coupled interference constraints. Our work resolves this problem for networks with primary interference constraints through the introduction of a novel fixed point formulation that approximates the link service rates of CSMA policies. The main contributions of the paper are as follows.

- We provide an analytical fixed-point formulation to approximate the performance of asynchronous CSMA poli- 
cies operating in multi-hop networks subject to collisions with primary interference constraints. Our formulation makes interesting connections to work by Hajek and Krishna on the accuracy of the Erlang fixed point for stochastic loss networks [17], [20]. While our technical development focuses on the primary interference model, we note that it suggests a general approach that can be used to handle higher-order interference models.

- We rigorously show that our fixed point formulation to approximate the performance of asynchronous CSMA policies is asymptotic accurate under an appropriate limiting regime where the network size becomes large. We also demonstrate through simulation results that such accuracy is achieved for moderately sized network. This is especially important since it suggests that the approximation will be useful even in realistic networks.

- We utilize the fixed-point formulation to characterize the achievable rate region of our CSMA policies, and further provide a constructive method to find the transmission attempt probabilities of a CSMA policy that can stably support a given network load in the achievable rate region. To the best of our knowledge, this constitutes the first such characterization of CSMA achievable rate region in multi-hop networks with the explicit incorporation of collisions.

- We show that for large networks with a balanced traffic load, the CSMA achievable rate region takes an extremely simple form that simply limits the individual load on each node to 1 , which is the maximum supportable load. This result together with the previous shows that the capacity region of large multi-hop wireless networks (asymptotically) takes on a very simple form.

The rest of the paper is organized as follows. We start by noting several relevant works in the context of CSMA policies in Section II. In Section III, we define our system model, and in Section IV we describe the class of CSMA policies we consider in this paper. In Section $\mathrm{V}$ we provide a summary and discussion of our main result, as well as an overview of the analysis. We provide our fixed point formulation and prove its asymptotic accuracy in Sections VI and VIII, respectively. Then, in Section VII and IX, we provide a characterization of the achievable rate region of the class of CSMA policies, and show that it is asymptotically capacity achieving. We end with concluding remarks in Section $\mathrm{X}$.

\section{RELATED WORK}

In this section, we provide a summary of the work on CSMA policies for single-hop and multihop networks that is most relevant to the analysis presented in this paper, and note the key differences of our work in this paper.

For single-hop networks where all nodes are within transmission range of each other, the performance of CSMA policies is well-understood [3]. Furthermore, the well-known "infinite node" approximations provides a simple characterization for the throughput of a given CSMA policy, as well as the achievable rate region of CSMA policies, in the case of a single-hop networks [3]. This approximation has been instrumental in the understanding of the performance of CSMA policies, as well as for the design of practical protocols for wireless local area networks. For the case where nodes are saturated and always have a packet to sent, the achievable rate region of CSMA policies is easily obtained [5]. For the case where nodes only make a transmission attempt when they have a packet to transmit has also recently been studied [5], [28].

For general multihop networks, results for CSMA policies are available for idealized situation of instantaneous channel feedback. This assumption of instantaneous channel feedback allows the elimination of collisions, which significantly simplifies the analysis, and allows the use of Markov chains to model system operation. Under such an instant feedback assumption, an early work [4] has shown that the stationary distribution of the associated Markov chain takes a product form. A more recent work [18] has utilized such a productform to derive a dynamic CSMA policy that, combined with rate control, achieves throughput-optimality while satisfying a given fairness criterion. Similar results with the same instantaneous feedback assumption have been independently derived in [33] in the context of optical networks and later extend to wireless networks [29]. Another relevant recent work [27] suggests a way of handling collisions under the synchronous CSMA operation.

Our approach differs from much of this literature in that we do not assume instantaneous feedback or time synchronization, and explicitly consider collisions, which are unavoidable in a real implementation. The incorporation of possible collisions require the development of a completely different modeling of the CSMA performance than the continuous-time Markov chain model used for the aforementioned idealized setup. Instead, we develop a novel fixed-point approximation for a specific interference model, and show its asymptotic accuracy.

An important byproduct of this development is the quantification of the proximity of the CSMA achievable rate region to the limiting capacity region as a function of the sensing period level. Such information will be extremely helpful in determining how small the sensing period should be to achieve a desired fraction of the capacity region.

Clearly, a non-zero sensing period, however small, must be considered in the CSMA operation to account for the propagation delay associated with transmissions. Yet, the inclusion of such a factor creates non-zero probabilities of collisions. Thus, in order to keep the collision level at a small level, the aggressiveness of the CSMA policy must depend on the particular value of the sensing period for the given system. In our development, we explicitly determine this connection and provide a constructive method to determine the CSMA parameters as a function of the sensing period. Moreover, in this paper we consider a completely asynchronous CSMA operation, which relaxes any synchronism assumptions amongst the nodes that will facilitate its practical implementation. Such a relaxation creates many technical challenges, which are resolved in this paper.

\section{SySTEM MODEL}

Network Model: We consider a fixed wireless network composed of a set $\mathcal{N}$ of nodes with cardinality $N$, and a set $\mathcal{L}$ of 
directed links with cardinality $L$. A directed link $(i, j) \in \mathcal{L}$ indicates that node $i$ is able to send data packets to node $j$. We assume that the rate of transmission is the same for all links and all packets are of a fixed length. Throughout the paper we rescale time such that the time it takes to transmit one packet is equal to one time unit.

For a given node $i \in \mathcal{N}$, let $\mathcal{U}_{i}:=\{j \in \mathcal{N}:(j, i) \in \mathcal{L}\}$ be the set of upstream nodes, i.e. the set containing all nodes from which $i$ can receive packets. Similarly, let $\mathcal{D}_{i}:=\{j \in \mathcal{N}$ : $(i, j) \in \mathcal{L}\}$ be set of downstream nodes, i.e. the set containing all nodes $j$ which can receive packets from $i$. Collectively, we denote the set of all the neighbors of node $i$ as $\mathcal{N}_{i}:=\mathcal{U}_{i} \cup \mathcal{D}_{i}$. Also, we let $\mathcal{L}_{i}:=\left\{(i, j): j \in \mathcal{D}_{i}\right\}$ be the set of outgoing links from node $i$, i.e. the set of all links from node $i$ to its downstream nodes $\mathcal{D}_{i}$ (see Fig. 1 for an example).

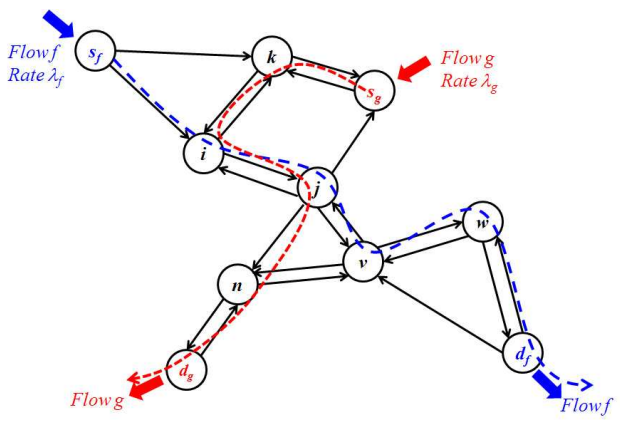

Fig. 1. Example of a network where two routes $f$ and $g$ given by $\mathcal{R}_{f}=\left\{\left(s_{f}, i\right),(i, j),(j, v),(v, w),\left(w, d_{f}\right)\right\}$ and $\mathcal{R}_{g}=$ $\left\{\left(s_{g}, k\right),(k, i),(i, j),(j, n),\left(n, d_{g}\right)\right\}$. In this network: the set of upstream neighbors of node $j$ is given by $\mathcal{U}_{j}=\{i, v\}$; the set of downstream neighbors of node $j$ is given by $\mathcal{D}_{j}=\left\{i, s_{g}, n, v\right\}$; the set of outgoing links of node $j$ is given by $\mathcal{L}_{j}=\left\{(j, i),\left(j, s_{g}\right),(j, v),(j, n)\right\}$; the set of links that interfere with $(i, j)$ is given by $\mathcal{I}_{(i, j)}=$ $\left\{(j, i),\left(s_{f}, i\right),(i, k),(k, i),\left(j, s_{g}\right),(j, v),(v, j),(j, n)\right\}$; the mean rate on link $(i, j)$ is given by $\lambda_{(i, j)}=\lambda_{f}+\lambda_{g}$; and the load on node $i$ is $\Lambda_{i}=2 \lambda_{f}+2 \lambda_{g}$.

Throughout the paper, we assume that $\mathcal{U}_{i}=\mathcal{D}_{i}$, for all $i \in \mathcal{N}$ so that we have $\mathcal{U}_{i}=\mathcal{D}_{i}=\mathcal{N}_{i}$, for each $i \in \mathcal{N}$. This assumption simplifies the notation as we can use a single set $\mathcal{N}_{i}$ to represent both $\mathcal{D}_{i}$ and $\mathcal{U}_{i}$. Our analysis can be extended to the more general case requiring only notational changes. Thus, henceforth we will describe a network by the tuple $(\mathcal{N}, \mathcal{L})$.

Interference Model: We focus on networks under the wellknown primary interference, or node exclusive interference, model [21], [40], defined next.

Definition 1 (Primary Interference Model). A packet transmission over link $(i, j) \in \mathcal{L}_{i}$ is successful if only if within the transmission duration ${ }^{1}$ there exists no other activity over any other link $(m, n) \in \mathcal{L}$ which shares a node with $(i, j)$. For each link $l \in \mathcal{L}$, we use $\mathcal{I}_{l}$ denote the set of links $l^{\prime} \in \mathcal{L}$ that interfere with link $l$, i.e. the set of all links $l^{\prime} \in \mathcal{L}$ that have a node in common with link $l$.

The primary interference model applies, for example, to wireless systems where multiple frequencies/codes are avail-

\footnotetext{
${ }^{1}$ Notice that our definition of interference model does not require a time slotted operation of the communication attempts, and hence applies to asynchronous network operation.
}

able (using FDMA or CDMA) to avoid interference, but each node has only a single transceiver and hence can only send to or receive from one other node at any time (see [31], [7] for additional discussion).

Traffic Model: We characterize the network traffic by a rate vector $\lambda:=\left\{\lambda_{r}\right\}_{r \in \mathcal{R}}$ where $\mathcal{R}$ is the set of routes used by the traffic, and $\lambda_{r}, \lambda_{r} \geq 0$, is the mean rate in packets per unit time along route $r \in \mathcal{R}$. For a given route $r \in \mathcal{R}$, let $s_{r}$ be its source node and $d_{r}$ be its destination node, and let

$$
\mathcal{R}_{r}=\left\{\left(s_{r}, i\right),(i, j), \cdots,(v, w),\left(w, d_{r}\right)\right\} \subset \mathcal{L}
$$

be the set of links traversed by the route. We allow several routes to be defined for a given source and destination pair $(s, d), s, d \in \mathcal{N}$.

Given the rate vector $\lambda=\left\{\lambda_{r}\right\}_{r \in \mathcal{R}}$, we let

$$
\lambda_{(i, j)}:=\sum_{r:(i, j) \in \mathcal{R}_{r}} \lambda_{r}, \quad(i, j) \in \mathcal{L},
$$

be the mean packet arrival rate to link $(i, j)$. Similarly, we let

$$
\Lambda_{i}(\lambda):=\sum_{j \in \mathcal{N}_{i}}\left[\lambda_{(i, j)}+\lambda_{(j, i)}\right], \quad i \in \mathcal{N} .
$$

be the mean packet arrival rate to node $i \in \mathcal{N}$ (see Figure 1 for an example).

To keep the notation light, we will in the following at times use the notation $\Lambda_{i}$ instead of $\Lambda_{i}(\lambda)$.

\section{POlicy SPACE ANd CSMA POLICY DESCRIPTION}

In this section, we introduce the space of scheduling policies that we are interested in, and provide the description of CSMA policies that we consider. We also define the notions of stability and achievable rate region that we use for our analysis.

\section{A. Scheduling Policies and Capacity Region}

Consider a fixed network $(\mathcal{N}, \mathcal{L})$ with traffic vector $\lambda=$ $\left\{\lambda_{r}\right\}_{r \in \mathcal{R}}$. A scheduling policy $\pi$ then defines the rules that are used to schedule packet transmissions on each link $(i, j) \in \mathcal{L}$. In the following, we focus on policies $\pi$ that have well-defined link service rates as a function of the rate vector $\lambda=\left\{\lambda_{r}\right\}_{r \in \mathcal{R}}$.

Definition 2 (Service Rate). For a given network $(\mathcal{N}, \mathcal{L})$, the offered service rate $\mu_{(i, j)}^{\pi}(\lambda)$ for link $l=(i, j) \in \mathcal{L}$ under policy $\pi$ and traffic vector $\lambda=\left\{\lambda_{r}\right\}_{r \in \mathcal{R}}$ is equal to the fraction of time that policy $\pi$ allocates for successfully transmitting packets on link $l=(i, j)$ under the primary interference model, i.e. the fraction of time node $i$ can send packets on link $l=(i, j)$ that will not experience interference from any link $l^{\prime} \in \mathcal{I}_{l}$.

Let $\mathcal{P}$ be the class of all policies $\pi$ that have well-defined link service rates. Note that this class contains a broad range of scheduling policies, including dynamic policies such as queuelength-based policies that are variations of the MaxWeight policy [38], as well as noncausal policies that know the future arrival of the flows. We then define network stability as follows. 
Definition 3 (Stability). For a given network $(\mathcal{N}, \mathcal{L})$, let $\mu^{\pi}(\lambda)=\left\{\mu_{(i, j)}^{\pi}(\lambda)\right\}_{(i, j) \in \mathcal{L}}$ be the vector of link service rates of policy $\pi, \pi \in \mathcal{P}$, for the rate vector $\lambda=\left\{\lambda_{r}\right\}_{r \in \mathcal{R}}$. We say that policy $\pi$ stabilizes the network for $\lambda$ if $\lambda_{(i, j)}<\mu_{(i, j)}^{\pi}(\lambda)$, $(i, j) \in \mathcal{L}$.

This commonly used stability criteria [38] requires that for each link $(i, j)$ the link service rate $\mu_{(i, j)}^{\pi}(\lambda)$ is larger than the arrival rate $\lambda_{(i, j)}$. The capacity region of a network $(\mathcal{N}, \mathcal{L})$ is then defined as follows.

Definition 4 (Capacity Region). For a given network $(\mathcal{N}, \mathcal{L})$, the capacity region $\mathcal{C}$ is equal to the set of all traffic vectors $\lambda=\left\{\lambda_{r}\right\}_{r \in \mathcal{R}}$ such that there exists a policy $\pi \in \mathcal{P}$ that stabilizes the network for $\lambda$, i.e. we have

$\mathcal{C}=\left\{\lambda \geq 0: \exists \pi \in \mathcal{P}\right.$ with $\left.\lambda_{(i, j)}<\mu_{(i, j)}^{\pi}(\lambda), \forall(i, j) \in \mathcal{L}\right\}$.

\section{B. CSMA Policies}

In this paper, we are interested in characterizing the performance of CSMA policies that operate by actively sensing the channel activity and, when idle, performing random transmission attempts according to the parameters of the particular CSMA policy. Before we describe the details of CSMA policy operation in Definition 6, we present our modeling of heterogeneous channel sensing delay that must exist in the real-world implementation of such policies.

Definition 5 (Sensing Delay $\left.\left\{\beta_{l}\left(l^{\prime}\right)\right\}\right)$. Consider a given link $l=(i, j) \in \mathcal{L}$. When a link $l^{\prime}$ in the interference region $\mathcal{I}_{l}$ of a link $l$ becomes idle (or busy), then transmitting node $i$ of link $l$ will not be able to detect this instantaneously, but only after some delay, to which we refer to as the sensing delay ${ }^{2}$ $\beta_{l}\left(l^{\prime}\right)$.

We note that the sensing delay given in the above definition is lower-bounded by the propagation delay between node $i$ and $i^{\prime}$. The exact length of the sensing delay will depend on the specifics of the sensing mechanism deployed. In Appendix A, we describe two possible approaches to how channel sensing could be performed for networks with primary interference constraints.

While the sensing delay of different node-link pairs may differ, throughout this work, we make the assumption that all sensing delays are bounded by a constant $\beta$ measured with respect to the normalized packet transmission duration. We refer to this upper bound $\beta$ as the sensing (or idle) period of a CSMA policy.

Assumption 1. There exists a constant $\beta$ to which we refer to as the sensing (or idle) period of a CSMA policy such that for all links $l \in \mathcal{L}$, we have that

$$
\beta_{l}\left(l^{\prime}\right) \leq \beta, \quad l^{\prime} \in \mathcal{I}_{l} .
$$

Recall that throughout the paper we rescale the time such that the time it takes to transmit one packet is equal to one

\footnotetext{
${ }^{2}$ In our subsequent discussion, for ease of exposition we will typically refer to links as performing sensing or scheduling a packet transmission. This must be understood as the transmitting node of the (directed) link performing the action.
}

time unit. Hence, the duration of an idle period $\beta$ is measured relative to the length of one packet transmission, i.e. if the length of an idle period is $L_{i}$ seconds and the length of a packet transmission is $L_{p}$ seconds, then we have $\beta=L_{i} / L_{p}$. For a fixed $L_{i}$, the duration of an idle period $\beta$ will become small if we increase the packet lengths. Hence, we can control the value of $\beta$ by modifying $L_{p}$ for a fixed $L_{i}$.

Definition 6 (CSMA(p, $\beta$ ) Policy). A CSMA policy is given by a transmission attempt probability vector $\mathbf{p}=\left(p_{(i, j)}\right)_{(i, j) \in \mathcal{L}} \in$ $[0,1]^{L}$ and a sensing period (or idle period) $\beta>0$, that satisfies Assumption 1.

Given $\mathbf{p}$ and $\beta$, the policy works as follows: each node, say $i$, senses the activity on its outgoing links $l \in \mathcal{L}_{i}$. We say that $i$ has sensed link $(i, j) \in \mathcal{L}_{i}$ to be idle for a duration of an idle period $\beta$ if for the duration of $\beta$ time units we have that (a) node $i$ has not sent or received a packet and (b) node $i$ has sensed that node $j$ has not sent or received a packet. If node $i$ has sensed link $(i, j) \in \mathcal{L}_{i}$ to be idle for a duration of an idle period $\beta$, then $i$ starts a transmission of a single packet on link $(i, j)$ with probability $p_{(i, j)}$, independent of all other events in the network. If node $i$ does not start a packet transmission, then link $(i, j)$ has to remain idle for another period of $\beta$ time units before $i$ again has the chance to start a packet transmission. Thus, the epochs at which node $i$ has the chance to transmit a packet on link $(i, j)$ are separated by periods of length $\beta$ during which link $(i, j)$ is idle, and the probability that $i$ starts a transmission on link $(i, j)$ after the link has been idle for $\beta$ time units is equal to $p_{(i, j)}$.

In the event that the idle periods of two links $l$ and $l^{\prime}$ that both originate at node $i$ end at the same time, we use the following mechanism to prevent the possibility that node $i$ starts in this case a transmission on both links $l$ and $l^{\prime}$ simultaneously (leading to sure collision): letting $\hat{\mathcal{L}}_{i}(t)$ denote the set of links in $\mathcal{L}_{i}$ for which an idle period ends at time $t$, for each link $l=(i, j) \in \hat{\mathcal{L}}_{i}(t)$ the probability that node $i$ starts a transmission on link $l$ at time $t$ is given by $\left(p_{(i, j)}\right) /\left(\sum_{\left\{j^{\prime}:\left(i, j^{\prime}\right) \in \hat{\mathcal{L}}_{i}(t)\right\}} p_{\left(i, j^{\prime}\right)}\right)$, independently of all other attempts by any node in the network.

Finally, we assume that packet transmission attempts are made according to above description regardless of the availability of packets at the transmitter. In the event of the absence of a data packet, the transmitting node transmits a dummy packet, which is discarded at the receiving end of the transmission (see also our discussion in Section X), but is counted in the service rate provided to that link.

We note that while all the nodes use the same sensing time $\beta$ to detect whether a given link is idle, the actual time that it takes a node to detect that another node has stopped (or started) transmitting a packet is determined by its individual sensing delay as given in Definition 5, which can be different for different nodes. Different sensing delays will lead to an asynchronous operation of the network where the sensing and packet transmission periods of different nodes are not aligned.

Also note that, under our CSMA policy, links make a transmission attempt with a fixed probability after the channel has been sensed to be idle, independent of the current backlog 
of the link. This may seem to be an unreasonable scenario as it implies that a link might make a transmission attempt even if there is no packet to be transmitted. However, there are at least two reasons why this situation is of interest. First, such a policy could indeed be implemented (where links send dummy packets once in a while) Second, and more importantly, being able to characterize the throughput of such a policy opens up the possibility of studying more complex, dynamic CSMA policies where the attempt probabilities depend on the current backlog. In particular, the results of our analysis can be used to formulate a fluid-flow model for backlog-dependent policies, where the instantaneous throughput at a given state (backlog vector) is given by the expected throughput obtained in our analysis. Such policies are of interest as they might allow for dynamic adaptation of the traffic load in the network (e.g. see [23]).

Given the length of an idle period $\beta$, in the following we will simply use $\mathbf{p}$ to refer to the $\operatorname{CSMA}(\mathbf{p}, \beta)$ policy. Next, we define the achievable rate region of a CSMA policy.

\section{Achievable Rate Region of CSMA Policies}

We show in Appendix C-F that a CSMA policy $\mathbf{p}$ has a welldefined link service rate vector to which we refer as $\mu(\mathbf{p})=$ $\left\{\mu_{(i, j)}(\mathbf{p})\right\}_{(i, j) \in \mathcal{L}}$, i.e. CSMA policies are contained in the set $\mathcal{P}$. Note that for a given $\beta$, the link service rate under a CSMA policy depends only on the transmission attempt probability vector $\mathbf{p}$, and not on the arrival rates $\lambda$. The achievable rate region of CSMA policies is then given as follows.

Definition 7 (Achievable Rate Region of CSMA Policies). For a given network $(\mathcal{N}, \mathcal{L})$ and a given sensing period $\beta$, the achievable rate region of CSMA policies is given by the set of rate vectors $\lambda=\left\{\lambda_{r}\right\}_{r \in \mathcal{R}}$ for which there exists a CSMA policy $\mathbf{p}$ that stabilizes the network for $\lambda$, i.e. we have that $\lambda_{(i, j)}<\mu_{(i, j)}(\mathbf{p}),(i, j) \in \mathcal{L}$.

\section{Overview of the Main Results And AnAlysis}

This section provides an overview of the main results of this work along with an outline of the analysis.

In Section IX, we derive an approximation $\Gamma(\beta)$ for the achievable rate region of CSMA policies for a given network and a given sensing period $\beta$, and show that in the limit as the sensing period $\beta$ approaches 0 we have that

$$
\lim _{\beta \downarrow 0} \Gamma(\beta)=\left\{\lambda \geq \mathbf{0}: \Lambda_{i}(\lambda)<1, \text { for all } i \in \mathcal{N}\right\} .
$$

Since it is impossible for any policy to stabilize the network if for a node $i$ we have that $\Lambda_{i}(\lambda) \geq 1$, this result suggest that in the limiting regime as $\beta$ becomes small, the capacity region for scheduling policies in wireless networks with primary interference constraints includes all rate vectors $\lambda$ such that

$$
\Lambda_{i}(\lambda)<1, \quad i \in \mathcal{N}
$$

We verify this intuition for large networks with many small flows, i.e. we show that asymptotic achievable rate region of CSMA policies under the limiting regime large networks with many small flows and a small sensing is of the above form.
We will provide a precise description of the limiting regime that we consider in Section IX.

The result that the achievable rate region of CSMA policies is asymptotically such that it can support any rate vector $\lambda$ satisfying (3) may seem very surprising and counter-intuitive at first. And indeed, it is important to stress that our result does not state that the achievable rate region of CSMA policies is always of the form as given by (3), but only under the conditions that (a) $\beta$ becomes small and (b) the network resources are shared by many small flows. Let us briefly comment on these two conditions.

The fact that $\beta$ needs to be small in order to obtain a large achievable rate region is rather intuitive; clearly if $\beta$ is large (let's say close to 1 ) then the above result will not be true. The fact that we need the assumption of many small flows in order to obtain our result is illustrated by the following example.

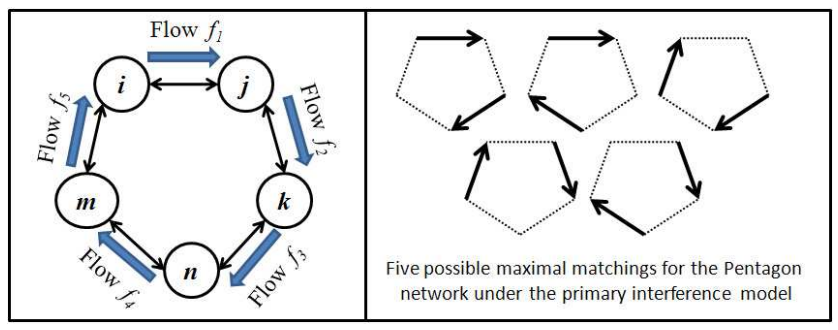

Fig. 2. The pentagon network with flows $r_{1}, \cdots, r_{5}$ on each link, and the five possible simultaneous transmissions that can occur under the primary interference model. The rate $\lambda_{r_{i}}=(1-\epsilon) / 2, i=1, \cdots, 5$, for any $\epsilon \in$ $(0,0.1]$ is not achievable by any policy for this scenario.

Example 1. For the pentagon network of Figure 2, let $\epsilon \in$ $(0,0.1]$ and $\lambda_{r_{i}}=(1-\epsilon) / 2$ for each $r=1, \cdots, 5$. Then, the load on each node is given by $\Lambda_{i}=(1-\epsilon)$ for each $i \in \mathcal{N}$. Although the resulting traffic vector $\lambda$ satisfies $E q$. (3), no scheduling policy can stabilize the network for $\lambda$. This can be seen by noting that at most two links out of five can transmit successfully at a given time, as shown in the figure. Hence, even an optimal centralized controller cannot achieve a maximum symmetric node activity of more than $2 / 5$, and clearly, our result cannot hold for this network.

The reason that in the pentagon network a node cannot achieve a throughput of more than $2 / 5$ is that under each "maximal" schedule given in Figure 2, if one of the neighboring nodes of a given node $i$ is busy transmitting, then node $i$ has to wait for a duration of 1 time unit to get a chance to make a transmission attempt. However, if we have a network where each node $i$ has many neighbors with which it exchanges data packets (many flows), then nodes will typically have to wait for much less than 1 time unit before they get the chance to start a packet transmissions. Intuitively, the larger the number of neighbors of a node, the shorter a node has to wait until it gets a chance to start a packet transmission. In addition to having many flows, we need the assumption that each flow is small in order to avoid the situation where the dynamics at each node is basically determined by a small number of large flows, essentially leading to a similar behavior as in the case where each node has only a small numbers 
of neighbors. Note however that these assumptions aren't sufficient in order to obtain our result; we also need to show that there exists a CSMA policy under which nodes (a) do not wait too long before making a transmission attempt (and hence waste bandwidth), (b) are not too aggressive such that a large fraction of packet transmissions result in collisions, and (c) share the available network resources such that the resulting link service rates indeed support a given traffic vector $\lambda$ that satisfies Eq. (3).

Below, we provide a brief description of the different steps taken in our analysis. Our first step is to derive a tractable formulation to characterize the link service rates for a given CSMA policy. Specifically, inspired by the reduced load approximations utilized in the loss network analysis [20], in Section VI-B we propose a novel fixed point formulation to model the performance of a CSMA policy $\mathbf{p}$. Similar to the reduced load approximation in loss networks, the fixed point equation is based on an independence assumption. We show that the fixed point is well-defined, i.e., there exists a unique fixed point. Our second step is to use the CSMA fixed point to characterize the approximate achievable rate region in Section VII, and show that this characterization suggests that CSMA policies are throughput-optimal in the limit as the sensing time $\beta$ becomes small. In our third step, we show that the formulated CSMA fixed point is asymptotically accurate in the sense that it accurately characterizes the link service rates of a CSMA policy as $\beta$ becomes small for large networks with many small flows. A technical issue that requires care in the proof is the scaling with which the sensing delay $\beta$ decays as a function of the network size $N$. We identify a proper scaling, as given in Assumption 2 of Section VIII, that yields the asymptotic accuracy result. Moreover, in the derivation of the achievable rate region using the CSMA fixed point, we obtain an algorithm that allows the constructive computation of the CSMA policy parameters that stabilize the network for any given rate vector $\lambda$ within the achievable rate region. Finally, in Section IX, we derive the asymptotic achievable rate region of CSMA policies for the limiting regime of large networks with many small flows and a small sensing period. This result shows that in this asymptote the CSMA achievable rate region can be described by a condition in the form of (3).

\section{Approximate CSMA Fixed Point Formulation}

In the first part of our analysis, we introduce a fixed point approximation, called the CSMA fixed point, to characterize the link service rates under a CSMA policy $\mathbf{p}$. The fixed point approximation extends the well-known infinite node approximation for single-hop networks (see for example [3]) to multihop networks which we briefly review below.

In the following we will use $\tau$ to denote the services rates obtained under our analytical formulations that we use to approximate the actual service rates $\mu(\mathbf{p})$ under a CSMA policy $\mathbf{p}$ as defined in Section IV-C.

\section{A. Infinite Node Approximation for Single-Hop Networks}

Consider a single-hop network where $N$ nodes share a single communication channel, i.e. where nodes are all within transmission range of each other. In this case, a CSMA policy is given by the vector $\mathbf{p}=\left(p_{1}, \cdots, p_{N}\right) \in[0,1]^{N}$ where $p_{n}$ is the probability that node $n$ starts a packet transmission after an idle period of length $\beta$ [3].

Suppose that the single-hop network is synchronized, i.e. the sensing delay is the same for all node pairs $n, n^{\prime} \in \mathcal{N}$ and we have that

$$
\beta_{i}(j)=\beta_{k}(l), \quad i, j, k, l \in \mathcal{N} .
$$

Then the network throughput, i.e. the fraction of time the channel is used to transmit packets that do not experience a collision, can then be approximated by (see for example [3])

$$
\tau(G(\mathbf{p}))=\frac{G(\mathbf{p}) e^{-G(\mathbf{p})}}{\beta+1-e^{-G(\mathbf{p})}}
$$

where $G(\mathbf{p})=\sum_{n=1}^{N} p_{n}$. Note that $G(\mathbf{p})$ captures the expected number of transmissions attempt after an idle period under a CSMA policy $\mathbf{p}$.

This well-known approximation is based on the assumption that a large (infinite) number of nodes share the communication channel. It is asymptotically accurate as the number of nodes $N$ becomes large and each node makes a transmission attempt with a probability $p_{n}, n \in \mathcal{N}$ that approaches zero while the offered load $G=\sum_{n=1}^{N} p_{n}$ stays constant (see for example [3]).

The following results are well-known. For $\beta>0$, one can show that

$$
\tau(G)<1, \quad G \geq 0,
$$

and for $G^{+}(\beta)=\sqrt{2 \beta}, \beta>0$, we have that

$$
\lim _{\beta \downarrow 0} \tau\left(G^{+}(\beta)\right)=1 \text {. }
$$

Using (4), the service rate $\mu_{n}(\mathbf{p})$ of node $n$ under a given CSMA policy $\mathbf{p}$ can be approximated by

$$
\tau_{n}(\mathbf{p})=\frac{p_{n} e^{-G(\mathbf{p})}}{1+\beta-e^{-G(\mathbf{p})}}, \quad n=1, \ldots, N .
$$

In the above expression, $p_{n}$ is the probability that node $n$ tries to capture the channel after an idle period and $e^{-G(\mathbf{p})}$ characterizes the probability that this attempt is successful, i.e. the attempt does not collide with an attempt by any other node.

Similarly, the fraction of time that the channel is idle can be approximated by

$$
\rho(\mathbf{p})=\rho(G(\mathbf{p}))=\frac{\beta}{\beta+1-e^{-G(\mathbf{p})}},
$$

where we have that $\lim _{\beta \downarrow 0} \rho\left(G^{+}(\beta)\right)=0$.

\section{B. CSMA Fixed Point Approximation for Multihop Networks}

We extend the above approximation for single-hop networks to multihop networks that operate in an asynchronous manner as described in Section IV-B as follows.

For a given a sensing period $\beta$, we approximate the fraction of time $\rho_{i}(\mathbf{p})$ that node $i$ is idle under the CSMA policy $\mathbf{p}$ by the following fixed point equation,

$$
\rho_{i}(\mathbf{p})=\frac{\beta}{\left(\beta+1-e^{-G_{i}(\mathbf{p})}\right)}, \quad i=1, \cdots, N,
$$


where

$$
G_{i}(\mathbf{p})=\sum_{j \in \mathcal{N}_{i}}\left[p_{(i, j)}+p_{(j, i)}\right] \rho_{j}(\mathbf{p}), \quad i=1, \cdots, N
$$

Note that the fixed point equation can be given both in terms of the fraction of idle times $\rho$ by substituting (10) in (9) or in terms of the transmission attempt rates $G$ by substituting (9) in (10). Given this equivalence, we refer to either one as the CSMA fixed point equation. We further let $\rho(\mathbf{p})=$ $\left(\rho_{1}(\mathbf{p}), \cdots, \rho_{N}(\mathbf{p})\right)$ and $G(\mathbf{p})=\left(G_{1}(\mathbf{p}), \cdots, G_{N}(\mathbf{p})\right)$ denote particular CSMA fixed points, and $\mathscr{R}(\mathbf{p})$ and $\mathscr{G}(\mathbf{p})$ denote the set of all fixed points of (9) and (10), respectively.

The intuition behind the CSMA fixed point equation (9) and (10) is as follows: suppose that the fraction of time that node $i$ is idle under the CSMA policy $\mathbf{p}$ is equal to $\rho_{i}(\mathbf{p})$, and suppose that the times when node $i$ is idle are independent of the processes at all other nodes. If node $i$ has been idle for $\beta$ time units, i.e. node $i$ has not received or transmitted a packet for $\beta$ time units, then node $i$ can start a transmission attempt on link $(i, j), j \in \mathcal{N}_{i}$, only if node $j$ also has been idle for an idle period of $\beta$ time units. Under the above independence assumption, this will be (roughly) the case with probability $\rho_{j}(\mathbf{p})$, and the probability that node $i$ start a packet transmission on the link $(i, j), j \in \mathcal{N}_{i}$, given that it has been idle for $\beta$ time units is (roughly) equal to $p_{(i, j)} \rho_{j}(\mathbf{p})$. Similarly, the probability that node $j \in \mathcal{N}_{i}$ starts a packet transmission on the link $(j, i)$ after node $i$ has been idle for $\beta$ time units is (roughly) equal to $p_{(j, i)} \rho_{j}(\mathbf{p})$. Hence, the expected number of transmission attempts that node $i$ makes or receives, after it has been idle for $\beta$ time units is (roughly) given by (10). Using (8) of Section VI-A, the fraction of time that node $i$ is idle under $\mathbf{p}$ can then be approximated by (9).

There are two important questions regarding the CSMA fixed point approximation. First, one needs to show that the CSMA fixed point is well-defined, i.e. that there always exists a unique CSMA fixed point. In the above notation this corresponds to proving that the sets $\mathscr{R}(\mathbf{p})$ and $\mathscr{G}(\mathbf{p})$ have a single element for any feasible $\mathbf{p}$. To that end, the following result, proven in Appendix B, establishes the uniqueness of a fixed point solution for all such $\mathbf{p}$.

Theorem 1. For every CSMA policy $\mathbf{p} \in(0,1)^{L}$, each of the set of fixed point solutions $\mathscr{R}(\mathbf{p})$ and $\mathscr{G}(\mathbf{p})$ has a single element, denoted henceforth by $\rho(\mathbf{p})$ and $G(\mathbf{p})$, respectively.

Second, we need to check the accuracy of the above CSMA fixed point approximation. This is postponed to Section VIII, where we show that the CSMA fixed point approximation is asymptotically accurate for large networks with a small sensing period $\beta$ and appropriately decreasing link attempt probabilities. In what follows, we focus on the CSMA achievable rate region characterization based on the above fixed point approximation.

\section{Approximate CSMA Achievable Rate Region}

In this section, we use the CSMA fixed point approximation (9) and (10) to characterize an approximate achievable rate region of CSMA policies. In Section IX, we will show that this characterization is asymptotically accurate for large networks with many small flows and a small sensing time, $\beta$.

We start by noting that, for a given sensing period $\beta$, we can use the CSMA fixed point $G(\mathbf{p})$ for a policy $\mathbf{p}$ to approximate the actual link service rate $\mu_{(i, j)}(\mathbf{p})$ under the CSMA policy $\mathbf{p}$ by $\tau_{(i, j)}(\mathbf{p})$ that satisfies

$$
\tau_{(i, j)}(\mathbf{p})=\frac{p_{(i, j)} \rho_{j}(\mathbf{p}) e^{-\left(G_{i}^{R}(\mathbf{p})+G_{j}(\mathbf{p})\right)}}{1+\beta-e^{-G_{i}(\mathbf{p})}}
$$

where

$$
G_{i}^{R}(\mathbf{p}) \triangleq \sum_{j \in \mathcal{N}_{i}} p_{(j, i)} \rho_{j}(\mathbf{p})
$$

represents the rate at which node $i$ receives transmission attempts by its neighbors, and hence its difference from $G_{i}(\mathbf{p})$.

Note that the above equation is similar to (7) where $p_{(i, j)} \rho_{j}(\mathbf{p})$ captures the probability that node $i$ makes an attempt to capture link $(i, j)$ if it has been idle for $\beta$ time units, and $\exp \left[-\left(G_{i}^{R}(\mathbf{p})+G_{j}(\mathbf{p})\right)\right]$ is the probability that this attempt is successful, i.e. the attempt does not overlap with an attempt by another link that shares a node with $(i, j)$. Note that

$$
\tau_{(i, j)}(\mathbf{p}) \geq \frac{p_{(i, j)} \beta e^{-\left(G_{i}(\mathbf{p})+G_{j}(\mathbf{p})\right)}}{\left(1+\beta-e^{-G_{i}(\mathbf{p})}\right)\left(1+\beta-e^{-G_{j}(\mathbf{p})}\right)}
$$

as $G_{i}(\mathbf{p}) \geq G_{i}^{R}(\mathbf{p})$.

The next result provides an approximate achievable rate region of the CSMA policy based on the CSMA fixed point approximation and the approximate service rates $\left(\tau_{(i, j)}(\mathbf{p})\right)_{(i, j)}$ given in (11).

Theorem 2. Given a network $(\mathcal{N}, \mathcal{L})$ with sensing period $\beta>$ 0 , let $\Gamma(\beta)$ be given by

$\Gamma(\beta) \triangleq\left\{\lambda \geq 0 \mid \Lambda_{i}(\lambda)<\tau\left(G^{+}(\beta)\right) e^{-\left(G^{+}(\beta)\right)}, \quad \forall i \in \mathcal{N}\right\}$,

where $G^{+}(\beta) \triangleq \sqrt{2 \beta}, \tau\left(G^{+}(\beta)\right)$ is as defined in (4), and $\Lambda_{i}(\lambda) \triangleq \sum_{j \in \mathcal{N}_{i}}\left[\lambda_{(i, j)}+\lambda_{(j, i)}\right]$, for each $i \in \mathcal{N}$.

Then, for every $\lambda \in \Gamma(\beta)$, we can explicitly find (cf. Equation (14)) a CSMA policy parameter $\mathbf{p}$ for which the corresponding CSMA fixed point approximation yields

$$
\lambda_{(i, j)}<\tau_{(i, j)}(\mathbf{p}), \quad(i, j) \in \mathcal{L},
$$

where $\tau_{(i, j)}(\mathbf{p})$ is as defined in (11). In other words, by a proper selection of $\mathbf{p}$, the approximate service rates can be made to exceed the traffic load on each link as long as $\lambda \in$ $\Gamma(\beta)$.

Proof: For brevity, we will denote $\Lambda_{i}(\lambda)$ as $\Lambda_{i}$, which, by definition, satisfies $\Lambda_{i}<\tau\left(G^{+}(\beta)\right) e^{-G^{+}(\beta)}$ for all $i \in \mathcal{N}$. For each node $i=1, \ldots, N$, choose $G_{i} \in\left[0, G^{+}(\beta)\right)$ such that

$$
e^{\left(G_{i}-G^{+}(\beta)\right)} \tau\left(G_{i}\right) e^{-G^{+}(\beta)}=\Lambda_{i}
$$

and let

$$
\rho_{i}=\frac{\beta}{\beta+1-e^{-G_{i}}} .
$$

Such a $G_{i}$ exists since the function

$$
f\left(G_{i}\right)=e^{\left(G_{i}-G^{+}(\beta)\right)} \tau\left(G_{i}\right) e^{-G^{+}(\beta)}
$$


is continuous in $G_{i}$ with $f(0)=0$ and

$$
f\left(G^{+}(\beta)\right)=\tau\left(G^{+}(\beta)\right) e^{-G^{+}(\beta)}>\Lambda_{i} .
$$

Using $\rho_{i}$ for $i=1, \ldots, N$ as defined above, consider the CSMA policy $\mathbf{p}$ given by

$$
p_{(i, j)}=\frac{\lambda_{(i, j)}}{\rho_{i} \rho_{j}} \beta e^{2 G^{+}(\beta)}, \quad(i, j) \in \mathcal{L} .
$$

By applying the above definitions, at every node $i=1, \ldots, N$ we have that

$$
\begin{aligned}
\sum_{j \in \mathcal{N}_{i}}\left[p_{(i, j)}+p_{(j, i)}\right] \rho_{j}=\sum_{j \in \mathcal{N}_{i}} \frac{\lambda_{(i, j)}+\lambda_{(j, i)}}{\rho_{i} \rho_{j}} \beta e^{2 G^{+}(\beta)} \rho_{j} \\
=\frac{\beta e^{2 G^{+}(\beta)}}{\rho_{i}} \sum_{j \in \mathcal{N}_{i}}\left[\lambda_{(i, j)}+\lambda_{(j, i)}\right]=\frac{\beta e^{2 G^{+}(\beta)}}{\rho_{i}} \Lambda_{i} \\
=\frac{\beta e^{2 G^{+}(\beta)}}{\rho_{i}} e^{\left(G_{i}-G^{+}(\beta)\right)} \tau\left(G_{i}\right) e^{-G^{+}(\beta)}=\beta \frac{e^{G_{i}}}{\rho_{i}} \tau\left(G_{i}\right) \\
=\beta \frac{\beta+1-e^{-G_{i}}}{\beta} e^{G_{i}} \frac{G_{i} e^{-G_{i}}}{\beta+1-e^{-G_{i}}}=G_{i} .
\end{aligned}
$$

This implies that the above choices of $G=\left(G_{1}, \cdots, G_{N}\right)$ and $\rho=\left(\rho_{1}, \cdots, \rho_{N}\right)$ define the CSMA fixed point of the static CSMA policy given by (14), i.e. we have that

$$
\rho(\mathbf{p})=\rho \text { and } G(\mathbf{p})=G .
$$

Using (12), the service rate $\tau_{(i, j)}(\mathbf{p})$ on link $(i, j)$ under $\mathbf{p}$ is then given by

$$
\begin{aligned}
& \tau_{(i, j)}(\mathbf{p}) \geq \frac{p_{(i, j)} \rho_{j}(\mathbf{p}) e^{-\left(G_{i}(\mathbf{p})+G_{j}(\mathbf{p})\right)}}{1+\beta-e^{-G_{i}(\mathbf{p})}} \\
& \quad=p_{(i, j)} \frac{\rho_{j} e^{-\left(G_{i}+G_{j}\right)}}{1+\beta-e^{-G_{i}}}=\frac{\lambda_{(i, j)}}{\rho_{i} \rho_{j}} \beta e^{2 G^{+}(\beta)} \frac{\rho_{j} e^{-\left(G_{i}+G_{j}\right)}}{1+\beta-e^{-G_{i}}} \\
& =\lambda_{(i, j)} \frac{\beta}{\rho_{i}\left(1+\beta-e^{\left.-G_{i}\right)}\right.} e^{2 G^{+}(\beta)-\left(G_{i}+G_{j}\right)} \\
& =\lambda_{(i, j)} e^{2 G^{+}(\beta)-\left(G_{i}+G_{j}\right)}>\lambda_{(i, j)},
\end{aligned}
$$

where we used in the last inequality the fact that by construction we have $G_{i}, G_{j}<G^{+}(\beta)$. The proposition then follows.

The proof of Theorem 2 is constructive in the sense that given a rate vector $\lambda \in \Gamma(\beta)$, we construct (cf. Equation (14)) a CSMA policy $\mathbf{p}$ such that $\lambda_{(i, j)}<\tau_{(i, j)}(\mathbf{p}),(i, j) \in \mathcal{L}$. We will use this construction for our numerical results in Section IX-C. Theorem 2 also leads to the following interesting corollary, which indicates the capacity achieving nature of CSMA policies in the small sensing delay regime.

Corollary 1. In the small sensing delay regime, i.e. as $\beta \downarrow 0$, the approximate achievable rate region $\Gamma(\beta)$ converges to the following simple set

$$
\lim _{\beta \downarrow 0} \Gamma(\beta)=\left\{\lambda \geq \mathbf{0} \mid \Lambda_{i}(\lambda)<1, i=1, \cdots, N\right\} .
$$

Proof: The proof follows immediately from the definition of $\Gamma(\beta)$ once we recall from Section VI-A that $\lim _{\beta \downarrow 0} G^{+}(\beta)=0$, and $\lim _{\beta \downarrow 0} \tau\left(G^{+}(\beta)\right)=1$.

Since any rate vector $\lambda$ for which there exists a node $i$ with $\Lambda_{i} \geq 1$ cannot be stabilized by any policy, Corollary 1 establishes that for networks with a small sensing time, the approximate achievable rate region of static CSMA policies get arbitrarily close to the above limiting rate region described purely in terms of per node traffic load. As we noted in Example 1, such a rate region is not achievable for all networks. In Section IX, we show that the capacity region does take on the above simple form for large networks with many small flows and a small sensing period $\beta$.

To that end, in the next section, we first establish conditions on the network and CSMA parameters for which CSMA fixed point approximation becomes accurate.

\section{Asymptotic CSMA FiXed PoInT ACCURACY}

In this section, we study the accuracy of the CSMA fixed point approximation proposed in Section VI (cf. Equations (9) and (10)) in capturing the service rate and idle fraction performance of the actual CSMA policy (cf. Definition 6). Our analysis establishes a large network and small sensing delay regime in which the approximation becomes arbitrarily accurate.

More precisely, we consider a sequence of networks for which the number of nodes $N$ increases to infinity, and let $\mathcal{L}^{(N)}$ and $\mathcal{N}_{i}^{(N)}$ respectively denote the set of all links and the set of neighbors of node $i$ for the network with $N$ nodes. Similarly, as $N$ increases, we consider a corresponding sequence of CSMA policies $\left\{\mathbf{p}^{(N)}\right\}_{N \geq 1}$ with a sequence of sensing periods $\left\{\beta^{(N)}\right\}_{N \geq 1}$, where $\left(\mathbf{p}^{(N)}, \beta^{(N)}\right)$ defines the CSMA policy for the network with $N$ nodes as described in Definition 6. We make the following assumptions on the parameters of the CSMA policy.

Assumption 2. For the sequences $\left\{\mathbf{p}^{(N)}\right\}_{N \geq 1}$ and $\left\{\beta^{(N)}\right\}_{N \geq 1}$ introduced above:

(a) $\lim _{N \rightarrow \infty} N \beta^{(N)}=0$.

(b) Letting $p_{\max }^{(N)} \triangleq \max _{(i, j) \in \mathcal{L}^{(N)}} p_{(i, j)}^{(N)}$, we have $\lim _{N \rightarrow \infty} \frac{p_{\text {max }}^{(N)}}{\beta^{(N)}}=$ 0.

(c) There exists a positive constant $\chi$ and a finite integer $N_{0}$, such that for all $N \geq N_{0}$ we have

$$
\sum_{j \in \mathcal{N}_{i}^{(N)}} \frac{\left[p_{(i, j)}^{(N)}+p_{(j, i)}^{(N)}\right]}{\beta^{(N)}} \leq \chi, \quad i=1, \cdots, N
$$

These technical assumptions have the following interpretation: Assumption 2(a) characterizes a small sensing delay regime by specifying how fast $\beta^{(N)}$ decreases to zero as the network size $N$ increases; Assumption 2(b) implies that the attempt probability of each link becomes small as $N$ becomes large, assuring that no single link dominates the service provided by its transmitting node; and Assumption 2(c) states that the total rate (given on the left of (15) by the expected number of transmission attempts per sensing period $\left.\beta^{(N)}\right)$ with which links incident to a given node $i$ start a packet transmission, is upper-bounded by a positive constant.

Below we provide two examples of networks that satisfy Assumption 2. 
Example 2. Consider an $N \times N$ switch (depicted in Figure 3) with traffic flowing from the set, $\mathcal{N}_{S}^{(N)}=\{1, \cdots, N\}$, of input (or sender) ports to the set, $\mathcal{N}_{R}^{(N)}=\{N+1, \cdots, 2 N\}$, of output (or receiver) ports. For this setup where the degree of each node is $N$, we can select the CSMA policy parameters as follows to satisfy the Assumption 2:

$$
\begin{aligned}
& \beta^{(N)}=1 /(N \log (N)), \quad \text { and } \\
& p_{(i, j)}=\chi \beta^{(N)} /(2 N), \quad \forall(i, j) \in \mathcal{N}_{S}^{(N)} \times \mathcal{N}_{R}^{(N)} .
\end{aligned}
$$

Example 3. Consider a network consisting of $N$ nodes and assume that each node communicates with $\log (N)$ neighboring nodes. This setup resembles randomly generated dense network within a unit area, where the nodes within the communication range of each other are connected. Such a model is widely studied in earlier works (e.g. [15]) that establish that if the communication radius is optimally selected for connectivity, the degree of each node scales as $\Theta(\log (N))$ for a network with $N$ nodes.

The following parameters as a function of the network size $N$ will satisfy Assumption 2:

$$
\begin{aligned}
& \beta^{(N)}=1 /(N \log (N)), \quad \text { and } \\
& p_{(i, j)} \leq \chi \beta^{(N)} /(\log (N)) \quad \forall(i, j) \in \mathcal{L}^{(N)} .
\end{aligned}
$$

Next, we analyze the accuracy of the CSMA fixed point approximation for the limiting regime given by Assumption 2, i.e. we let $\rho\left(\mathbf{p}^{(N)}\right)=\left(\rho_{1}\left(\mathbf{p}^{(N)}\right), \cdots, \rho_{N}\left(\mathbf{p}^{(N)}\right)\right)$ be the CSMA fixed point for the network of size $N$, and let $\sigma_{i}\left(\mathbf{p}^{(N)}\right)$ be the actual fraction of time that node $i$ is idle under the $\operatorname{CSMA}\left(\mathbf{p}^{(N)}\right)$ operation. Then, we use the following metric to measure the discrepancy of the two:

$$
\delta_{\rho}^{(N)} \triangleq \max _{i=1, \cdots, N}\left|\rho_{i}\left(\mathbf{p}^{(N)}\right)-\sigma_{i}\left(\mathbf{p}^{(N)}\right)\right|,
$$

which quantifies the maximum approximation error of the CSMA fixed point across the network. Similarly, we let $\tau_{(i, j)}\left(\mathbf{p}^{(N)}\right)$ be the approximate CSMA service rate for link $(i, j)$ defined in (11), and let $\mu_{(i, j)}\left(\mathbf{p}^{(N)}\right)$ be the actual CSMA service rate for link $(i, j)$. Then, we define the following metric to measure the discrepancy between the two:

$$
\delta_{\tau}^{(N)} \triangleq \max _{(i, j) \in \mathcal{L}^{(N)}}\left|1-\frac{\tau_{(i, j)}\left(\mathbf{p}^{(N)}\right)}{\mu_{(i, j)}\left(\mathbf{p}^{(N)}\right)}\right|,
$$

which quantifies the maximum relative approximation error of the link service rates under the CSMA fixed point. Note that under Assumption 2 the link service rate $\mu_{(i, j)}\left(\mathbf{p}^{(N)}\right)$ will approach zero as $N$ increases and the error term $\mid \tau_{(i, j)}\left(\mathbf{p}^{(N)}\right)-$ $\mu_{(i, j)}\left(\mathbf{p}^{(N)}\right) \mid$ will trivially vanish; this is the reason why we consider the relative error when studying the accuracy of the CSMA fixed point equation for the link service rates.

The following result, proven in Appendix C, establishes that in the limit as $N$ approaches infinity, the fixed point approximation for CSMA polices with the above scaling becomes asymptotically accurate.

Theorem 3. Under the CSMA policy scaling of Assumption 2, we have that

$$
\lim _{N \rightarrow \infty} \delta_{\rho}^{(N)}=0, \quad \text { and } \quad \lim _{N \rightarrow \infty} \delta_{\tau}^{(N)}=0,
$$

i.e., the fixed point approximation becomes asymptotically accurate both in terms of idle fraction and service rate approximations.

\section{A. Numerical Results}

In this section, we illustrate Theorem 3 using numerical results obtained for the $N \times N$ switch network discussed in Example 3 and depicted in Figure 3. The switch topology is selected for numerical comparison since such a topology is the simplest non-trivial one that also leads to an analytically tractable fixed point solution under symmetric conditions. Yet, we emphasize that Theorem 3 applies to any large network as long as CSMA policy satisfies Assumption 2. Besides confirming the asymptotic accuracy of the approximations, our results also indicate that the accuracy is observed even for relatively small networks.

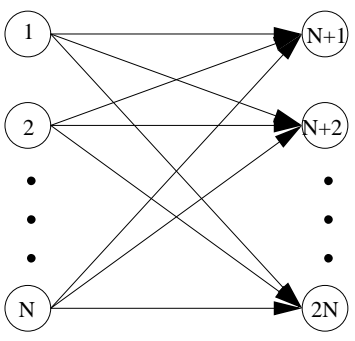

Fig. 3. Network topology for our numerical results consists of a set of $N$ sender nodes $\mathcal{N}_{S}^{(N)}=\{1, \ldots, N\}$, and a set of $N$ receiver nodes $\mathcal{N}_{R}^{(N)}=$ $\{N+1, \ldots, 2 N\}$. The set of links $\mathcal{L}^{(N)}$ consists of all directed links $(i, j)$ from a sender $i \in \mathcal{N}_{S}$ to a receiver $j \in \mathcal{N}_{R}$.

For this network, we consider a sequence of CSMA policies $\mathbf{p}^{(N)}=\left(p_{(i, j)}^{(N)}\right)_{(i, j) \in \mathcal{L}^{(N)}}$ and the corresponding sequence of sensing periods $\beta^{(N)}$ as in (16) by setting $\chi=10$. Recall that a CSMA policy with parameters $\left(\mathbf{p}^{(N)}, \beta^{(N)}\right)$ determines the link probabilities $p_{(i, j)}^{(N)}$ with which sender $i \in \mathcal{N}_{S}^{(N)}$ starts a transmission of a packet to receiver $j \in \mathcal{N}_{R}^{(N)}$ after link $(i, j)$ has been sensed to be idle for sensing period of $\beta^{(N)}$ time units. Given a sensing period $\beta^{(N)}$, the CSMA fixed point for a policy $\mathbf{p}^{(N)}$ is then given by

$$
\rho_{i}\left(\mathbf{p}^{(N)}\right)=\frac{\beta^{(N)}}{\left(\beta^{(N)}+1-e^{-G_{i}\left(\mathbf{p}^{(N)}\right)}\right)}, \quad i=1, \cdots, 2 N,
$$

where

$$
\begin{aligned}
G_{i}\left(\mathbf{p}^{(N)}\right) & =\sum_{j \in \mathcal{N}_{R}^{(N)}} p_{(i, j)}^{(N)} \rho_{j}\left(\mathbf{p}^{(N)}\right), \quad i \in \mathcal{N}_{S}^{(N)}, \quad \text { and } \\
G_{j}\left(\mathbf{p}^{(N)}\right) & =\sum_{i \in \mathcal{N}_{S}^{(N)}} p_{(i, j)}^{(N)} \rho_{i}\left(\mathbf{p}^{(N)}\right), \quad j \in \mathcal{N}_{R}^{(N)} .
\end{aligned}
$$

Then, due the symmetry of the network topology as well as of the constructed CSMA policies $\mathbf{p}^{(N)}$, the CSMA fixed point $\rho\left(\mathbf{p}^{(N)}\right)$ is uniform and satisfies

$$
\rho_{i}\left(\mathbf{p}^{(N)}\right)=\rho_{j}\left(\mathbf{p}^{(N)}\right), \quad i, j \in \mathcal{N}^{(N)} \triangleq \mathcal{N}_{S}^{(N)} \cup \mathcal{N}_{R}^{(N)} .
$$

In Figures 4 and 5, we evaluate the performance of the above sequence of CSMA policies for varying size $N$ of the sender set $\mathcal{N}_{S}$. In particular, Figure 4 depicts the measured mean 

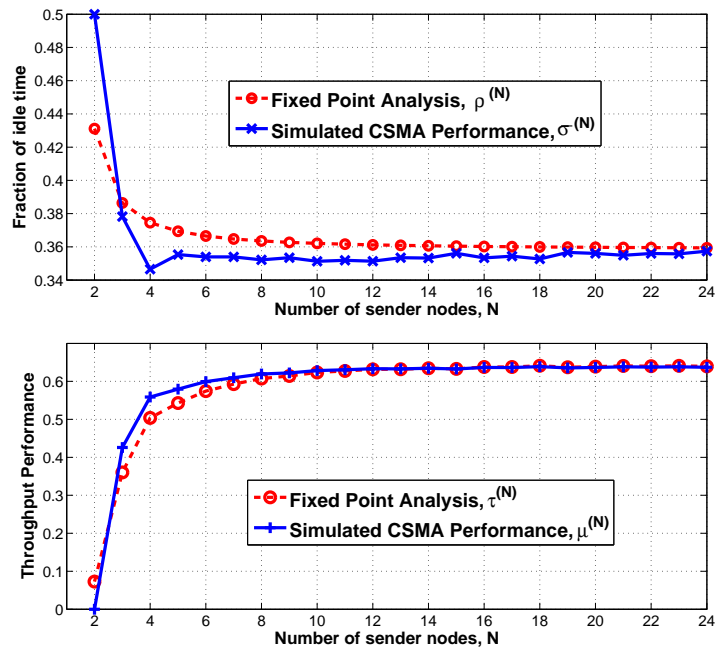

Fig. 4. Comparison of the actual fraction of idle time under the CSMA policy and the predicted values based on the fixed point formulation.
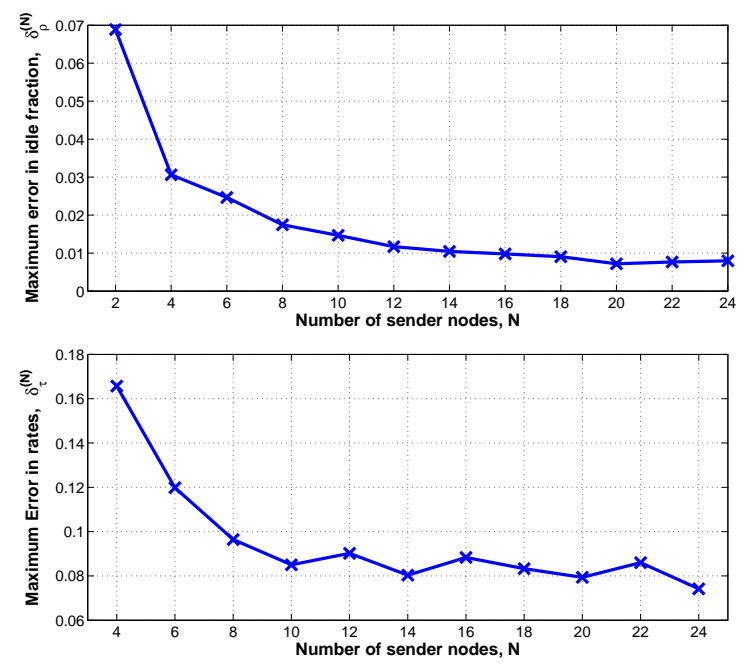

Fig. 5. Error terms of Theorem 3 for different values of $N$.

fraction of times that nodes are idle and mean node throughput under the actual CSMA policy operation, compared with the performance predicted by the CSMA fixed point. Figure 5 shows the error terms of Theorem 3 for the approximation error in the fraction of time that nodes are idle, and the link service rates.

Note that the above numerical results not only confirm the asymptotic claims of Theorem 3 but also indicate that the CSMA fixed point approximation is remarkably accurate even for smaller values of $N$. This suggests that the CSMA fixed point approximation may be used to characterize the performance for moderate-size networks where each nodes has a relatively small number of neighbors. An extensive investigation of this implication in more general network topologies is of practical interest and is left to future research.

\section{Asymptotic CAPACity Region $\mathcal{C}_{\infty}$}

In this section, we derive the asymptotic achievable rate region for CSMA for a limiting regime of large networks with many small flows and a small sensing period that is formally defined in Section IX-A.

\section{A. Many Small Flows Asymptotic}

In Section VIII, we introduced a sequence of networks for which the number of nodes $N$ increases to infinity, and let $\mathcal{L}^{(N)}$ be the set of all links in the network with $N$ nodes, and $\mathcal{N}_{i}^{(N)}$ be the set of neighbors of node $i$ in the network with $N$ nodes. In this section, we introduce a similar scaling for the traffic arrival rate vectors to assure that the load on any link do not dominate the load in its neighborhood. To that end, we use the notation $\lambda^{(N)}=\left\{\lambda_{r}^{(N)}\right\}_{r \in \mathcal{R}^{(N)}}$ for the arrival rate vector for the network with $N$ nodes. Furthermore,

$$
\begin{aligned}
\lambda_{(i, j)}^{(N)} & =\sum_{r \in \mathcal{R}^{(N)}:(i, j) \in r} \lambda_{r}^{(N)}, \quad(i, j) \in \mathcal{L}^{(N)}, \quad \text { and } \\
\Lambda_{i}^{(N)} & =\sum_{j \in \mathcal{N}_{i}^{(N)}}\left[\lambda_{(i, j)}^{(N)}+\lambda_{(j, i)}^{(N)}\right], \quad i \in \mathcal{N}^{(N)},
\end{aligned}
$$

respectively, denotes the mean packet arrival rate on link $(i, j)$ and the mean packet arrival rate at node $i$.

Definition 8 (Many Small Flows Asymptotic). Given a sequence of networks $\left\{\mathcal{N}^{(N)}, \mathcal{L}^{(N)}\right\}_{N \geq 1}$, we define $\mathcal{A}$ as the set of all rate vector sequences $\left\{\lambda^{(N)}\right\}_{N \geq 1}$ such that

$$
\limsup _{N \rightarrow \infty}\left(\max _{(i, j) \in \mathcal{L}^{(N)}} \lambda_{(i, j)}^{(N)}\right)=0 .
$$

We say that $\left\{\lambda^{(N)}\right\}_{N \geq 1}$ satisfies the many small flows asymptotic if it belongs to $\mathcal{A}$.

The above definition characterizes the limiting regime where the mean arrival of each flow becomes small as the network size scales, i.e. the network traffic consists of many small flows. It is important to note that, while the load on each link vanishes under the many small flows asymptote, the total load on a node may be non-vanishing if the number of neighbors also increases. We shall see that this key characteristic of the many small flows regime will allow CSMA policies to achieve maximal per node loads under large and wellconnected network topologies. Before we establish this main result, we define the asymptotic achievable rate region of CSMA policies under the many small flows asymptotic as follows.

Definition 9 (Asymptotic CSMA Achievable Rate Region). The asymptotic achievable rate region of static CSMA policies under the many flow limit is the set of flow rate sequences $\left\{\lambda^{(N)}\right\}_{N \geq 1} \in \mathcal{A}$ for which there exists a sequence of CSMA scheduling policies $\left(\mathbf{p}^{(N)}, \beta^{(N)}\right)_{N \geq 1}$ such that

$$
\liminf _{N \rightarrow \infty}\left(\min _{(i, j) \in \mathcal{L}^{(N)}} \frac{\mu_{(i, j)}\left(\mathbf{p}^{(N)}\right)}{\lambda_{(i, j)}^{(N)}}\right)>1 .
$$

Thus, every flow rate sequence $\left\{\lambda^{(N)}\right\}_{N \geq 1}$ in the asymptotic CSMA rate region can be stabilized by the sequence of CSMA policies $\left(\mathbf{p}^{(N)}, \beta^{(N)}\right)_{N \geq 1}$ for large enough $N$. 
Note that a sequence $\left\{\lambda^{(N)}\right\}_{N \geq 1} \in \mathcal{A}$ for which there exists a node $i$ with

$$
\lim _{N \rightarrow \infty} \Lambda_{i}^{(N)} \geq 1
$$

cannot be stabilized by any policy as service rate at each node is bounded by 1 . Hence, the achievable region under the many flow limit is contained in the set

$\mathcal{C}_{\infty} \triangleq\left\{\left\{\lambda^{(N)}\right\}_{N} \in \mathcal{A} \mid \limsup _{N \rightarrow \infty}\left(\max _{i=1, \ldots, N} \Lambda_{i}^{(N)}\right)<1\right\}$.

We refer to $\mathcal{C}_{\infty}$ as the capacity region under the many small flows asymptotic.

\section{B. Asymptotic CSMA Achievable Rate Region}

In this subsection, we characterize the asymptotic achievable rate region of CSMA policies under the many small flows asymptotic for networks with a small sensing period. To do this, we again consider a sequence of sensing periods $\left\{\beta^{(N)}\right\}_{N>1}$ that satisfies Assumption 2(a). The next theorem, proven in Appendix D, shows that in this case the achievable rate region of CSMA policies converges to the capacity region under the many small flows asymptotic $\mathcal{C}_{\infty}$.

Theorem 4. Given a sequence of networks $\left\{\mathcal{N}^{(N)}, \mathcal{L}^{(N)}\right\}_{N \geq 1}$, a sequence of sensing periods $\left\{\beta^{(N)}\right\}_{N \geq 1}$ satisfying Assumption 2(a), and a sequence of flow rates $\left\{\lambda^{(N)}\right\}_{N \geq 1} \in \mathcal{C}_{\infty}$, we can explicitly find a sequence of CSMA policy attempt rates $\left\{\mathbf{p}^{(N)}\right\}_{N \geq 1}$ that asymptotically stabilizes the network, i.e., that satisfies

$$
\liminf _{N \rightarrow \infty}\left(\min _{(i, j) \in \mathcal{L}^{(N)}} \frac{\mu_{(i, j)}\left(\mathbf{p}^{(N)}\right)}{\lambda_{(i, j)}^{(N)}}\right)>1 .
$$

It is interesting to note that the proof of Theorem 4 in Appendix D is constructive in that sense that it provides explicit expressions for the link transmission attempt probabilities that stabilize the network for a given rate vector sequence $\left\{\lambda^{(N)}\right\}_{N \geq 1}$ in $\mathcal{C}_{\infty}$.

\section{Numerical Results}

In this section, we verify the statement of Theorem 4 using the same switch topology we used for the numerical results in Section VIII-A (see also Figure 3). As the network size increases, we consider a sequence of idle periods $\left\{\beta^{(N)}\right\}_{N \geq 1}=0.1 /(N \log (N))$ and traffic vectors $\left\{\lambda^{(N)}\right\}_{N \geq 1}$ with

$\lambda_{(i, j)}^{(N)}=\frac{0.95}{N} e^{-G^{+}\left(\beta^{(N)}\right)} \tau\left(G^{+}\left(\beta^{(N)}\right)\right), \quad i \in \mathcal{N}_{S}, j \in \mathcal{N}_{R}$.

Notice that $\left\{\lambda^{(N)}\right\}_{N \geq 1}$ satisfies the many small flows asymptotic (cf. Definition 8) and that the per node load satisfies

$$
\Lambda_{i}^{(N)}=0.95 \cdot e^{-G^{+}\left(\beta^{(N)}\right)} \tau\left(G^{+}\left(\beta^{(N)}\right)\right), \quad i \in \mathcal{N}^{(N)},
$$

which is non-vanishing. Also note that the selected rate vector $\lambda^{(N)}$ is within that approximate CSMA achievable rate region $\Gamma\left(\beta^{(N)}\right)$ (cf. Equation 13) for each $N$.

In the proof for Theorem 2 we derive an explicit construction for obtaining a policy $\mathbf{p}^{(N)}$ that supports a given traffic vector $\lambda \in \Gamma\left(\beta^{(N)}\right)$. Following this construction for the above choice of flow rates, we choose $G^{(N)} \in\left[0, G^{+}\left(\beta^{(N)}\right)\right)$ such that

$$
\begin{aligned}
e^{\left(G^{(N)}-G^{+}\left(\beta^{(N)}\right)\right)} \tau\left(G^{(N)}\right) e^{-G^{+}\left(\beta^{(N)}\right)} & \\
& =0.95 \cdot e^{-G^{+}\left(\beta^{(N)}\right)} \tau\left(G^{+}\left(\beta^{(N)}\right)\right),
\end{aligned}
$$

which is shown to exist in the proof. Then, letting

$$
\rho^{(N)} \triangleq \frac{\beta^{(N)}}{\beta^{(N)}+1-e^{-G^{(N)}}},
$$

we construct a sequence of CSMA policy parameters $\mathbf{p}^{(N)}$ satisfying

$$
p_{(i, j)}^{(N)} \triangleq \frac{\lambda_{(i, j)}^{(N)}}{\left(\rho^{(N)}\right)^{2}} \beta^{(N)} e^{2 G^{+}\left(\beta^{(N)}\right)}, \quad(i, j) \in \mathcal{L}^{(N)} .
$$

Theorem 4 then states that for such constructed sequence of CSMA policies we have, for a large enough $N$, that $\mu_{(i, j)}^{(N)}>$ $\lambda_{(i, j)}^{(N)}$, for all $(i, j) \in \mathcal{L}^{(N)}$. Also, noting that $\lim _{N \rightarrow \infty} \Lambda_{i}^{(N)}=$ 0.95 for the above choice of flow rates, we have

$$
\begin{aligned}
& \lim _{N \rightarrow \infty} \sum_{j \in \mathcal{N}_{R}^{(N)}} \mu_{(i, j)}^{(N)}>0.95, \quad i \in \mathcal{N}_{S}^{(N)}, \quad \text { and } \\
& \lim _{N \rightarrow \infty} \sum_{i \in \mathcal{N}_{S}^{(N)}} \mu_{(i, j)}^{(N)}>0.95, \quad j \in \mathcal{N}_{R}^{(N)} .
\end{aligned}
$$

To confirm these asymptotic claims and to investigate their correctness for moderate values of $N$ we simulate the above network to measure the true link service rates for increasing $N$. Figure 6 shows the average node throughput that we obtained. Note that the average node throughput indeed is above the value $\Lambda^{(N)}$ for which we designed the CSMA policy $\mathbf{p}^{(N)}$. Furthermore, as $N$ increases the average node throughput becomes larger then 0.95 as predicted by our theoretical result. Moreover, these results indicate that the results are quite accurate even for small network sizes and that CSMA policies can be close to capacity achieving even if the number of neighbors of each node is relatively small.

Figure 6 shows the distribution of the ratio of link service rates to link loads. We know from Theorem 4 that this ratio will eventually exceed 1 for all links as $N$ tends to infinity. We observe in Figure 6 that already at a moderate value of $N=20$, more than $95 \%$ of the links exceed 1 and the rest of the links achieve rates close to 1 .

\section{Conclusions}

In this work, we provided an extensive analysis of asynchronous CSMA policies operating in multi-hop wireless networks subject to collisions with primary interference constraints. To that end, we first introduced a CSMA fixedpoint formulation to:(a) approximate the performance of such CSMA policies; (b) approximate their achievable rate region; and (c) provide a constructive method for determining the transmission attempt probabilities of the CSMA policy that can support a given rate vector in the achievable rate region.

We then showed that the CSMA fixed point formulation becomes asymptotically accurate for an appropriate limiting 

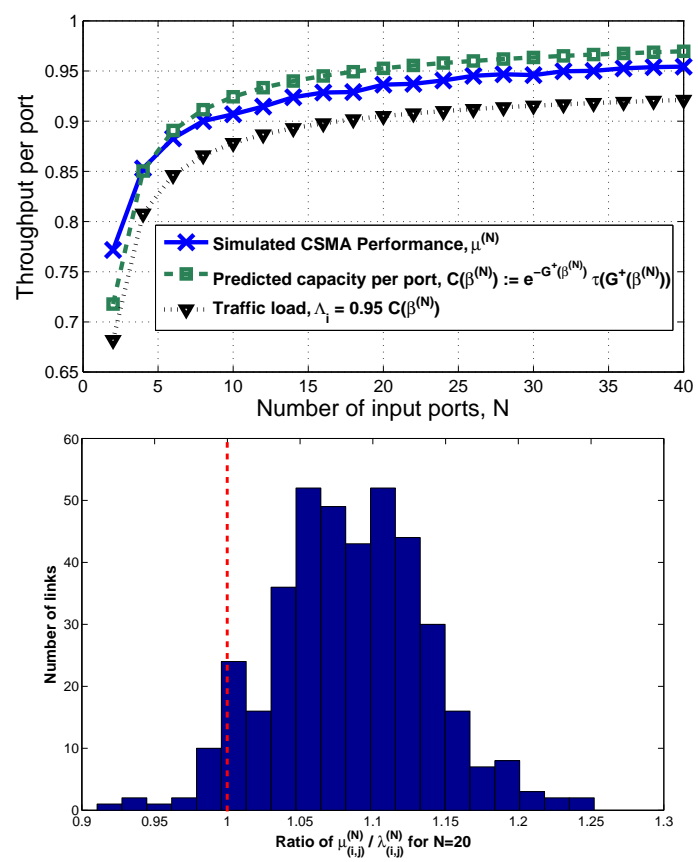

Fig. 6. Performance of the CSMA policy for the network in Figure 3 with symmetric load. The graph on the left shows that the policy achieves rates close to the aimed value of 0.95 per sender node even for moderate values of $N$. The graph on the right shows the distribution of the ratio of achieved rates to load on each link amongst 400 existing links in the network in Figure 3 with $N=20$

regime where the network size increases and the sensing delay decreases. Using this result we established that for large networks with a balanced traffic load, the CSMA achievable rate region takes an extremely simple form that simply limits the individual load on each node to 1 , which is the maximum supportable load by any other scheduling policy. This result has proven not only that the class of asynchronous CSMA policies is asymptotically throughput-optimal, but also that the capacity region of such large networks takes an extremely simple form, describable by per node loads.

Despite the asymptotic nature of our theoretical results, our simulation results have indicated that the CSMA fixed point approximate is remarkably accurate even for moderately sized network, which suggests that the approximation is useful for realistic network topologies.

\section{ACKNOWLEDGEMENT}

We thank Mahdi Lotfinezhad for correcting the proof of Theorem 4.

\section{REFERENCES}

[1] M. Andrews, K. Kumaran, K. Ramanan, A. Stolyar, R. Vijayakumar, and P. Whiting. Scheduling in a queueing system with asynchronously varying service rates, 2000. Bell Laboratories Technical Report.

[2] D. Bertsekas. Nonlinear Programming. Athena Scientific, Belmont, MA, 1995

[3] D. Bertsekas and R. Gallager. Data Networks. Prentice Hall, Englewood Cliffs, NJ, 1990

[4] R. Boorstyn, A. Kershenbaum, B. Maglaris, and V. Sahin. Throughput analysis in multi-hop csma packet radio networks. IEEE Transactions on Communications, 35(3):267-274, March 1987.
[5] C. Bordenave, D. McDonald, and A. Proutiere. Performance of random medium access - an asymptotic approach. 2008.

[6] L. Bui, A. Eryilmaz, R. Srikant, and X. Wu. Joint asynchronous congestion control and distributed scheduling for wireless networks. Proceedings of IEEE Infocom 2006.

[7] P. Chaporkar, K. Kar, and S. Sarkar. Throughput guarantees through maximal scheduling in wireless networks. In Proceedings of the Allerton Conference on Control, Communications and Computing, 2005.

[8] A. Eryilmaz, A. Ozdaglar, and E. Modiano. Polynomial complexity algorithms for full utilization of multi-hop wireless networks. 2007. Proceedings of IEEE Infocom.

[9] A. Eryilmaz, A. Ozdaglar, D. Shah, and E. Modiano. Distributed crosslayer algorithms for the optimal control of multi-hop wireless networks. submitted to IEEE/ACM Transactions on Networking, 2008.

[10] A. Eryilmaz, R. Srikant, and J. R. Perkins. Throughput optimal scheduling for broadcast channels. In Modelling and Design of Wireless Networks, Proceedings of SPIE, E. K. P. Chong (editor), volume 4531, pages 70-78, Denver, CO, 2001

[11] A. Eryilmaz, R. Srikant, and J. R. Perkins. Stable scheduling policies for fading wireless channels. IEEE/ACM Transactions on Networking, 13:411-425, April 2005.

[12] R. Gallager. Discrete Stochastic Processes. Springer, 1995.

[13] P. Giaccone, B. Prabhakar, and D. Shah. Randomized scheduling algorithms for high-aggregate bandwidhth switches. IEEE Journal on Selected Areas in Communications, 21(4):546-559, 2003.

[14] A. Gupta, X. Lin, and R. Srikant. Low-complexity distributed scheduling algorithms for wireless networks. In Proceedings of IEEE Infocom, 2007.

[15] P. Gupta and P. R. Kumar. The capacity of wireless networks. IEEE Transactions on Information Theory, IT-46(2):388-404, March 2000.

[16] P. Gupta and A. Stolyar. Optimal throughput allocation in general random-access networks. In CISS, Princeton, NJ, 2006.

[17] B. Hajek and A. Krishna. Bounds on the accuracy of the reduced-load blocking formula for some simple circuit-switched networks. In Proceedings of International Conference on New Trends in Communication, Control and Signal Processing, 1990.

[18] L. Jiang and J. Walrand. A csma distributed algorithm for throughput and utility maximization in wireless networks. 2008.

[19] C. Joo, X. Lin, and N. Shroff. Understanding the capacity region of the greedy maximal scheduling algorithm in multi-hop wireless networks. In Proceedings of IEEE INFOCOM, 2008.

[20] F. P. Kelly. Loss networks. The Annals of Applied Probability, 1(3):319378, August 1991.

[21] X. Lin and N. Shroff. The impact of imperfect scheduling on crosslayer rate control in multihop wireless networks. In Proceedings of IEEE Infocom, Miami, FL, March 2005.

[22] P. Marbach. Rate control in random access networks: The finite node case. In Proceedings of IEEE Conference on Decision and Control, Paradise Island, Bahamas, 2004.

[23] P. Marbach. Distributed scheduling and active queue management in wireless networks. 2007.

[24] P. Marbach and Y. Lu. Active queue management and scheduling for wireless networks: The single-cell case. In Proceedings of Conference on Information Sciences and System (CISS), 2006.

[25] E. Modiano, D. Shah, and G. Zussman. Maximizing throughput in wireless networks via gossiping. In ACM SIGMETRICS/IFIP Performance, 2006.

[26] M. Neely, E. Modiano, and C. Rohrs. Dynamic power allocation and routing for time varying wireless networks. In Proceedings of IEEE Infocom, pages 745-755, April 2003.

[27] J. Ni and R. Srikant. Distributed csma/ca algorithms for achieving maximum throughput in wireless networks, 2009. http://arxiv.org/abs/0901.2333.

[28] A. Proutiere, Y. Yi, and M. Chiang. Throughput of random access without message passing. In CISS, March 2008.

[29] S. Rajagopalan, D. Shah, and J. Shin. Aloha that works, 2008. submitted.

[30] S. Sanghavi, L. Bui, and R. Srikant. Distributed link scheduling with constant overhead, 2006. Technical Report.

[31] G. Sasaki and B. Hajek. Link scheduling in polynomial time. IEEE Transactions on Information Theory, 32:910-917, 1988.

[32] D. Shah. Stable algorithms for input queued switches. In Proceedings of the Allerton Conference on Control, Communications and Computing, 2001.

[33] D. Shah and R. Sreevastsa. Reversible networks, distributed optimization, and network scheduling: what do they have in common? 2008. 
[34] S. Shakkottai and A. Stolyar. Scheduling for multiple flows sharing a time-varying channel: The exponential rule. Translations of the AMS, Series 2, A volume in memory of F. Karpelevich, 207:185-202, 2002.

[35] A. Stolyar. Dynamic distributed scheduling in random access networks. Journal of Applied Probability, 45(2):297-313, 2008.

[36] L. Tassiulas. Scheduling and performance limits of networks with constantly varying topology. IEEE Transactions on Information Theory, 43:1067-1073, May 1997.

[37] L. Tassiulas. Linear complexity algorithms for maximum throughput in radio networks and input queued switches. In Proceedings of IEEE Infocom, pages 533-539, 1998.

[38] L. Tassiulas and A. Ephremides. Stability properties of constrained queueing systems and scheduling policies for maximum throughput in multihop radio networks. IEEE Transactions on Automatic Control, 36:1936-1948, December 1992.

[39] X. Wang and K. Kar. Cross-layer rate optimization in multi-hop wireless networks with random access. IEEE Journal on Selected Areas in Communications, 24:1548-1559, August 2006.

[40] X. Wu and R. Srikant. Regulated maximal matching: A distributed scheduling algorithm for multi-hop wireless networks with nodeexclusive spectrum sharing. In Proceedings of IEEE Conference on Decision and Control., 2005.

[41] X. Wu and R. Srikant. Bounds on the capacity region of multi-hop wireless networks under distributedgreedy scheduling. In Proceedings of IEEE Infocom, 2006.

[42] X. Wu, R. Srikant, and J. Perkins. Queue-length stability of maximal greedy schedules in wireless networks. IEEE Transactions on Mobile Computing, pages 595-605, June 2007.

\section{APPENDIX A \\ EXAmple Channel Sensing Mechanisms}

In this section, we discuss two specific channel sensing mechanisms that operate under heterogeneous sensing delay characteristics. We note that our model is flexible enough to allow other mechanism designs.

Mechanism 1: Suppose that each node $i \in \mathcal{N}$ is assigned a channel $c_{i}$ over which it receives data packets, and suppose that the sensing radius and transmission radius of the nodes are different. The channel $c_{i}$ could either be a frequency range, or a code, if a FDMA-based, or a CDMA-based, approach respectively is used to obtain a network with primary interference constraints (see also our discussion in Section III). Nodes that are within the transmission radius of a node can successfully receive its packet transmission if there are no collisions by another transmission within the transmission radius of the receiver. Nodes that are within the sensing radius of the transmitting node can only detect the presence or absence of activity together with its destination. The activity within the sensing radius does not cause collisions, but it signals the presence of activity. In this setting, a node $j \in \mathcal{N}_{i}$ can sense whether node $i$ is currently sending a packet by scanning the channels $c_{k}$ used by node $i$ for transmission on its outgoing links $(i, k) \in \mathcal{L}_{i}$. Furthermore, if the sensing radius is at least twice the transmission radius, then a node $j \in \mathcal{N}_{i}$ can sense whether node $i$ is currently receiving a packet by scanning channel $c_{i}$. Note that the time (measured in seconds) that it takes a node to detect whether a neighboring node is busy, will increase as the number of neighbors of a node increases; however, the sensing delay $\beta_{l}\left(l^{\prime}\right)$ measured relative to the time it takes to transmit a packet can still kept low by increasing the size of a packet, and hence increase the time $L_{p}$ it takes to transmit a packet.

Mechanism 2: Again, suppose that each node $i \in \mathcal{N}$ is assigned a communication channel $c_{i}$ over which it receives data

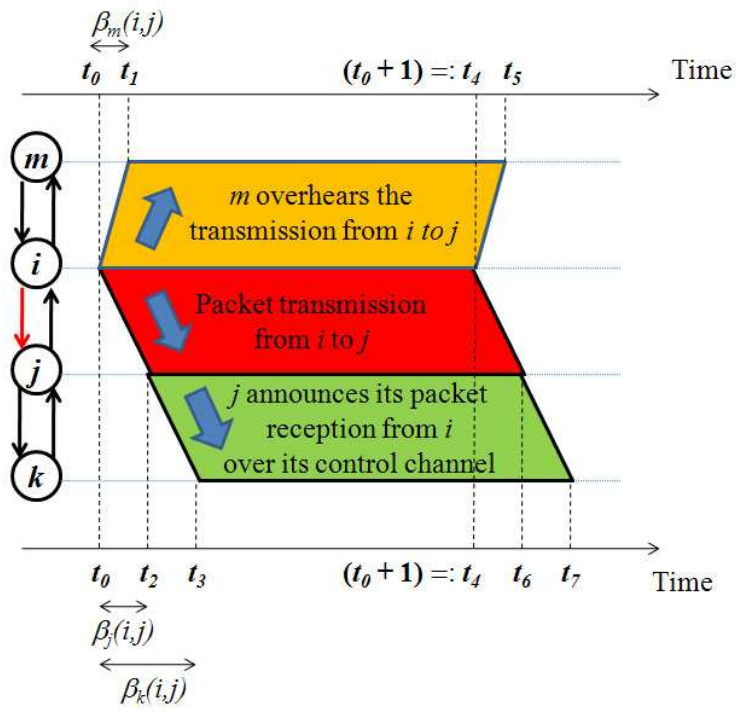

Fig. 7. Nodes $m, i, j$, and $k$ are connected as shown on the left. Node $i$ starts a packet transmission to node $j$ at $t_{0}$, which is overheard starting at $t_{1}$ by node $m$. Thus, the sensing delay $\beta_{m}(i, j)$ is equal to $\left(t_{1}-t_{0}\right)$. Node $j$ starts reception of the packet at $t_{2}$ (hence its sensing delay satisfies $\left.\beta_{j}(i, j)=\left(t_{2}-t_{0}\right)\right)$ and generates a signal over its control channel $\bar{c}_{j}$ to indicate the activity of link $(i, j)$. Node $k$ senses the control signal of node $j$ at time $t_{3}$ (hence its sensing delay is $\beta_{k}(i, j)=\left(t_{3}-t_{0}\right)$. The transmission of the packet ends at time $t_{4}$ which equals $\left(t_{0}+1\right)$ since the packet transmission duration is normalized to one. Nodes $m, j$, and $k$ sense the end of the activity at $t_{5}, t_{6}$, and $t_{7}$, respectively.

packets, and that in addition it is assigned a control channel $\bar{c}_{i}$, where the bandwidth of the communication channel $c_{i}$ is much larger than the one of the control channel $\bar{c}_{i}$. Then, if node $i$ is currently receiving a packet transmission on its communication channel $c_{i}$, then it can send out a busy signal on the control channel $\bar{c}_{i}$. In this setting, a node $j \in \mathcal{N}_{i}$ can sense whether node $i$ is currently sending a packet by scanning the channels $c_{k}$ used by node $i$ for transmission on its outgoing links $(i, k) \in \mathcal{L}_{i}$. Furthermore, a node $j \in \mathcal{N}_{i}$ can sense whether node $i$ is currently receiving a packet by scanning the control channel $\bar{c}_{i}$. Again, the time (measured in seconds) that it takes a node to detect whether a neighboring node is busy, will increase as the number of neighbors of a node increases; but the sensing delay $\beta_{l}\left(l^{\prime}\right)$ measured relative to the time it takes to transmit a packet can still kept low by increasing the size of a packet. Figure 7 gives a timing-diagram for this case.

\section{APPENDIX B}

\section{EXISTENCE AND UniQUENESS OF CSMA FiXed PoInTs}

In this section, we prove Theorem 1 which states that for each choice of $\mathbf{p} \in(0,1)^{L}$ there exists a unique CSMA fixed point. We first establish the existence of a CSMA fixed point.

Lemma 1. For every CSMA policy $\mathbf{p} \in[0,1]^{L}$, there exists a CSMA fixed point $\rho(\mathbf{p})$ and $G(\mathbf{p})$, i.e., the sets $\mathscr{R}(\mathbf{p})$ and $\mathscr{G}(\mathbf{p})$ are non-empty.

Proof: The proof uses the continuity properties of the fixed point equation given (9), and is a straightforward application of the Brouwer's fixed point theorem. 
We next establish the uniqueness of the CSMA fixed point for any $\mathbf{p} \in(0,1)^{L}$. Unlike standard methods in establishing the uniqueness of a fixed point, our proof method does not require additional assumptions on the fixed point mapping, therefore may be of independent interest. The proof follows a number of steps, which is outlined here for clarity: Proposition 1 shows the existence of a unique solution to the fixed point equation for a particular choice of $\hat{\mathbf{p}} \in(0,1)^{L}$, i.e., that $\mathscr{G}(\mathbf{p})=\{\hat{G}\}$ for some $\hat{G}$; Proposition 2 proves the upper-semicontinuity of the correspondence $\mathscr{G}(\mathbf{p})$ given by (10); Proposition 3 proves that for any CSMA policy $\mathbf{p}$ and $G \in \mathscr{G}(\mathbf{p}),(\mathbf{p}, G(\mathbf{p}))$ is uniquely defined in an open neighborhood of ( $\mathbf{p}, G(\mathbf{p}))$; finally Theorem 1 combines the preceding results to establish the global uniqueness of the CSMA fixed point for any $\mathbf{p} \in(0,1)^{L}$.

Proposition 1. For any network topology and any $\beta>0$, there exists a $\hat{\mathbf{p}} \in(0,1)^{L}$ for which there is a unique point $\hat{G} \in \mathscr{G}(\hat{\mathbf{p}})$ that solves the fixed point equation described in (9) and (10).

Proof: We restrict our choice of $\hat{\mathbf{p}}$ to the symmetric case of $\hat{p}_{(i, j)}=\hat{\theta} / 2$ for all $(i, j) \in \mathcal{L}$ and set $\hat{\theta}$ to any value in the non-empty range $\left(0, \zeta \frac{\beta}{d_{\max }}\right)$, where $d_{\max }$ denotes the maximum degree of the network and $\zeta$ is any positive constant strictly less than 1 . For this symmetric choice of link attempt probabilities, the fixed point equation (10) becomes:

$G_{i}(\hat{\mathbf{p}})=\sum_{j \in \mathcal{N}_{i}} \frac{\hat{\theta} \beta}{1+\beta-e^{-G_{j}(\hat{\mathbf{p}})}} \triangleq \hat{T}_{i}(G(\hat{\mathbf{p}})), \quad i=1, \cdots, N$,

which also introduces the mapping $\hat{T}_{i}: \mathbb{R}^{N} \rightarrow \mathbb{R}$ of $G(\hat{\mathbf{p}})$ to $G_{i}(\hat{\mathbf{p}})$ that must hold for any $G(\hat{\mathbf{p}}) \in \mathscr{G}(\hat{\mathbf{p}})$. More compactly, we can define the mapping $\hat{T}: \mathbb{R}^{N} \rightarrow \mathbb{R}^{N}$ as $\hat{T}(G) \triangleq\left(\hat{T}_{1}(G), \hat{T}_{2}(G), \cdots, \hat{T}_{N}(G)\right)$ and write the fixed point equation as $G(\hat{\mathbf{p}})=\hat{T}(G(\hat{\mathbf{p}}))$.

Next, we will show that the mapping $\hat{T}(\cdot)$ is a contraction mapping under the $l_{1}$ norm: $\|\mathbf{x}-\mathbf{y}\|_{1}=\sum_{i=1}^{N}\left|x_{i}-y_{i}\right|$ for $\mathbf{x}, \mathbf{y} \in \mathbb{R}^{N}$, which directly implies that the fixed point of the mapping is unique. For any two feasible vectors $G^{1}$ and $G^{2}$ with non-negative entries, we have

$$
\begin{aligned}
\| \hat{T}\left(G^{1}\right) & -\hat{T}\left(G^{2}\right) \|_{1} \\
= & \sum_{i=1}^{N}\left|\sum_{j \in \mathcal{N}_{i}}\left(\frac{\hat{\theta} \beta}{1+\beta-e^{-G_{j}^{1}}}-\frac{\hat{\theta} \beta}{1+\beta-e^{-G_{j}^{2}}}\right)\right| \\
& \leq \sum_{i=1}^{N} \sum_{j \in \mathcal{N}_{i}}\left|\frac{\hat{\theta} \beta}{1+\beta-e^{-G_{j}^{1}}}-\frac{\hat{\theta} \beta}{1+\beta-e^{-G_{j}^{2}}}\right| \\
& \stackrel{(a)}{\leq} \quad \frac{\hat{\theta}}{\beta} \sum_{i=1}^{N} \sum_{j \in \mathcal{N}_{i}}\left|G_{j}^{1}-G_{j}^{2}\right| \\
& \stackrel{(b)}{\leq} \frac{\hat{\theta} d_{\max }}{\beta} \sum_{i=1}^{N}\left|G_{j}^{1}-G_{j}^{2}\right| \\
& \stackrel{(c)}{\leq} \quad \zeta\left\|G^{1}-G^{2}\right\|_{1},
\end{aligned}
$$

which establishes that $\hat{T}$ is a contraction mapping, and therefore has a unique fixed point. To complete the proof, we justify the inequalities (a)-(c) in the above derivation. To get inequality (a), we note that the arising real function $h(z) \triangleq$ $\frac{\hat{\theta} \beta}{1+\beta-e^{-z}}$ is a decreasing convex function with $h^{\prime}(0)=-\hat{\theta} / \beta$, and hence satisfies $\left|h\left(z_{1}\right)-h\left(z_{2}\right)\right| \leq \frac{\hat{\theta}}{\beta}\left|z_{1}-z_{2}\right|$ for all $z_{1}, z_{2} \geq 0$. Inequality (b) follows from the fact that for each $i$, the difference $\left|G_{i}^{1}-G_{i}^{2}\right|$ appears at most $d_{\max }$ times in the previous double summation. Finally, inequality (c) follows from the assumption that $\hat{\theta} \in\left(0, \zeta \frac{\beta}{d_{\max }}\right)$.

We note that the proof of Proposition 1 can be slightly modified to establish that, as long as the link attempt probabilities are chosen sufficiently small, the fixed point equation has a unique solution. However, we shall take a different direction to show a stronger result that the uniqueness holds for any $\mathbf{p} \in(0,1)^{L}$, not only for sufficiently small values. To that end, we next study the continuity properties of $\mathscr{G}(\mathbf{p})$. The proof uses the continuity of the mapping

$$
f_{i}(G, \mathbf{p})=G_{i}-\sum_{j \in \mathcal{N}_{i}} \frac{\beta\left[p_{(i, j)}+p_{(j, i)}\right]}{\left(1+\beta-e^{-G_{j}}\right)}, \quad i=1, \ldots, N
$$

Note that for $f(G, \mathbf{p})=\left[f_{i}(G, \mathbf{p})\right]_{i=1, \ldots, N}$ we have that $f(G(\mathbf{p}), \mathbf{p})=\mathbf{0}$.

Proposition 2. The correspondence $\mathscr{G}:[0,1]^{L} \mapsto \mathbb{R}_{+}^{N}$ is upper-semicontinuous; i.e., $\mathscr{G}(\mathbf{p})$ has a closed graph.

Proof: Note that for all $\mathbf{p} \in[0,1]^{L}, \mathscr{G}(\mathbf{p})$ is given by

$$
\mathscr{G}(\mathbf{p})=\left\{G \in \mathbb{R}_{+}^{N} \mid f_{i}(G, \mathbf{p})=0, i=1, \ldots, N\right\} .
$$

We will show that $\mathscr{G}$ has a closed graph. Let $\left\{\left(\mathbf{p}_{k}, G_{k}\right)\right\}$ be a sequence which satisfies $G_{k} \in \mathscr{G}\left(\mathbf{p}_{k}\right)$ for all $k$ and converges to some $(\overline{\mathbf{p}}, \bar{G})$. Assume to arrive at a contradiction that $\bar{G} \notin \mathscr{G}(\overline{\mathbf{p}})$. By (20), this implies that there exists some $i \in\{1, \ldots, N\}$ such that $f_{i}(\bar{G}, \overline{\mathbf{p}}) \neq 0$. Assume without loss of generality that there exists some $\epsilon>0$ such that

$$
f_{i}(\bar{G}, \overline{\mathbf{p}})>2 \epsilon .
$$

By the continuity of the functions $f_{i}$, we have

$$
\lim _{k \rightarrow \infty} f_{i}\left(G_{k}, \mathbf{p}_{k}\right)=f_{i}(\bar{G}, \overline{\mathbf{p}})
$$

which implies the existence of some $\bar{K}$ such that

$$
\left|f_{i}(\bar{G}, \overline{\mathbf{p}})-f_{i}\left(G_{k}, \mathbf{p}_{k}\right)\right| \leq \epsilon, \quad \forall k \geq \bar{K} .
$$

Combined with (21), this yields

$$
f_{i}\left(G_{k}, \mathbf{p}_{k}\right) \geq f_{i}(\bar{G}, \overline{\mathbf{p}})-\epsilon>\epsilon,
$$

contradicting the fact that $G_{k} \in \mathscr{G}\left(\mathbf{p}_{k}\right)$ [cf. (20)].

Recall the definition of the mapping $f(G, \mathbf{p})=$ $\left[f_{i}(G, \mathbf{p})\right]_{i=1, \ldots, N}$ given by (19). The next proposition establishes the local uniqueness of the correspondence $\mathscr{G}(\mathbf{p})$.

Proposition 3. For all CSMA policies $\mathbf{p} \in(0,1)^{L}$ and all CSMA fixed points $G \in \mathscr{G}(\mathbf{p})$, there exist open neighborhoods $U \subset \mathbb{R}_{+}^{N}$ of $G$ and $V \subset(0,1)^{L}$ of $\mathbf{p}$ such that for each $\tilde{\mathbf{p}} \in V$ the equation $f(\tilde{G}, \tilde{\mathbf{p}})=0$ has a unique solution $\tilde{G} \in U$. Moreover, this solution can be given by a function $\tilde{G}=\phi(\tilde{\mathbf{p}})$ where $\phi$ is continuously differentiable on $V$. 
Proof: We prove this statement by using the implicit function theorem (see, e.g., [2]). For node $i \in \mathcal{N}$ we have

$$
\frac{\partial f_{i}}{\partial G_{j}}= \begin{cases}1 & i=j, \\ 0 & j \notin \mathcal{N}_{i}, \\ \psi_{(i, j)} \varphi_{j} & j \in \mathcal{N}_{i},\end{cases}
$$

with

$$
\begin{aligned}
\psi_{(i, j)} & \triangleq\left[p_{(i, j)}+p_{(j, i)}\right] \frac{\beta}{\beta+\left(1-e^{-G_{j}}\right)}, \quad \forall(i, j) \in \mathcal{L} ; \\
\varphi_{j} & \triangleq \frac{e^{-G_{j}}}{\beta+\left(1-e^{-G_{j}}\right)} \quad \forall j \in \mathcal{N}_{i} .
\end{aligned}
$$

Note that the function $f$ is continuously differentiable. Therefore, in order to use the implicit function theorem we need to show that the matrix

$$
\left[\left.\frac{\partial f_{i}}{\partial G_{j}}\right|_{G=G(\mathbf{p})}\right]
$$

has linearly independent rows. Before we proceed, we note that this matrix is a non-negative matrix.

Suppose that the rows are not linearly independent, then there exists a coefficient vector $\mathbf{x}=\left(x_{1}, \ldots, x_{N}\right) \neq \mathbf{0}$ such that

$$
\sum_{j=1}^{N} x_{j}\left(\frac{\partial f_{j}(\mathbf{p})}{\partial G_{i}}\right)=0, \quad \text { for all } i \in\{1, \ldots, N\} .
$$

Using the special structure of the Jacobian matrix, we obtain

$$
x_{i}+\varphi_{i} \sum_{j \in \mathcal{N}_{i}} \psi_{(j, i)} x_{j}=0, \quad \text { for all } i \in\{1, \ldots, N\}
$$

and

$$
x_{i}=-\varphi_{i} \sum_{j \in \mathcal{N}_{i}} \psi_{(j, i)} x_{j}, \quad \text { for all } i \in\{1, \ldots, N\} .
$$

Consider node $i^{*}$ such that for all $i=1, \ldots, N$ we have

$$
\left|x_{i^{*}}\left[\beta+\left(1-e^{-G_{i^{*}}}\right)\right]\right| \geq\left|x_{j}\left[\beta+\left(1-e^{-G_{j}}\right)\right]\right| .
$$

Then,

$$
\begin{aligned}
1 & =-\varphi_{i^{*}} \sum_{j \in \mathcal{N}_{i^{*}}} \psi_{\left(j, i^{*}\right)} \frac{x_{j}}{x_{i^{*}}} \\
\leq & \varphi_{i^{*}} \sum_{j \in \mathcal{N}_{i^{*}}}\left[p_{\left(i^{*}, j\right)}+p_{\left(j, i^{*}\right)}\right] \frac{\beta}{\beta+\left(1-e^{\left.-G_{i^{*}}\right)}\right.} \cdot \ldots \\
& \ldots \cdot \frac{\left|x_{j}\left[\beta+\left(1-e^{-G_{j}}\right)\right]\right|}{\left|x_{i^{*}}\left[\beta+\left(1-e^{-G_{j}}\right)\right]\right|} \\
& \stackrel{(a)}{\leq} \quad \varphi_{i^{*}} \sum_{j \in \mathcal{N}_{i^{*}}}\left[p_{\left(i^{*}, j\right)}+p_{\left(j, i^{*}\right)}\right] \frac{\beta}{\beta+\left(1-e^{\left.-G_{j}\right)}\right.} \\
& \stackrel{(b)}{=} \frac{G_{i^{*}}(\mathbf{p}) e^{-G_{i^{*}}(\mathbf{p})}}{\beta+\left(1-e^{-G_{i^{*}}(\mathbf{p})}\right)} \stackrel{(c)}{<} 1,
\end{aligned}
$$

where $(a)$ follows from (23), (b) follows from the fact that $f_{i^{*}}(G, \mathbf{p})=0$, and (c) follows from (5). This proves that the Jacobian matrix in (22) is non-singular. The result follows from the implicit function theorem.

We next combine Propositions 1-3 to complete the proof of Theorem 1.
Proof of Theorem 1: By Proposition 1, for the choice of $\hat{\mathbf{p}}$ in the proposition, there exists a unique fixed point $G(\hat{\mathbf{p}})$. For any given policy $\mathbf{p} \in(0,1)^{L}$ define the convex combination of $\hat{\mathbf{p}}$ and $\mathbf{p}$ as

$$
\overline{\mathbf{p}}(t)=(1-t) \hat{\mathbf{p}}+t \mathbf{p}, \quad t \in[0,1] .
$$

By Lemma 1, the set $\mathscr{G}(\overline{\mathbf{p}}(t))$ is nonempty, i.e., there exists at least one CSMA fixed point at $\overline{\mathbf{p}}(t)$ for each $t \in[0,1]$. We use the following lemma to complete the proof.

Lemma 2. For every $G \in \mathscr{G}(\mathbf{p})$, there exists a continuous function $\mathbf{h}:[0,1] \rightarrow \mathbb{R}_{+}^{N}$ that satisfies: $\mathbf{h}(0)=G(\hat{\mathbf{p}}) ; \mathbf{h}(t) \in$ $\mathscr{G}(\overline{\mathbf{p}}(t))$, for $t \in(0,1)$, where $\overline{\mathbf{p}}(t)$ is defined in (25); and $\mathbf{h}(1)=G$.

Proof: We define the set of functions

$\mathcal{H} \triangleq\left\{\mathbf{h}:[0,1] \rightarrow \mathbb{R}_{+}^{N} \mid \mathbf{h}(t) \in \mathscr{G}(\overline{\mathbf{p}}(t)), t \in[0,1] ; \mathbf{h}(1)=G\right\}$.

For each $\mathbf{h} \in \mathcal{H}$, let $\mathcal{T}_{\mathbf{h}} \in[0,1]$ denote the set of points at which $\mathbf{h}$ is discontinuous. Clearly, the set $\mathcal{T}_{\mathbf{h}}$ is either empty, or non-empty and bounded for each $\mathbf{h} \in \mathcal{H}$. To arrive at a contradiction, suppose $\mathcal{T}_{\mathbf{h}}$ is nonempty for some $\mathbf{h} \in \mathcal{H}$, and define the point $\tilde{t} \in[0,1]$ as

$$
\tilde{t} \triangleq \inf _{\left\{t \in \mathcal{T}_{\mathbf{h}}\right\}} t .
$$

Note that since $G(\overline{\mathbf{p}}(0))=G(\hat{\mathbf{p}})$ is unique (from Proposition 1) and $(\mathbf{p}, G(\mathbf{p}))$ is uniquely defined in a neighborhood of $(\hat{\mathbf{p}}, G(\hat{\mathbf{p}})$ (from Proposition 3), we must have $\tilde{t}>0$. Moreover, by the upper-semicontinuity of $\mathscr{G}(\mathbf{p})$ (cf. Proposition 2), the function $\mathbf{h}$ can be chosen to be right continuous at $\tilde{t}$, implying that $\mathbf{h}$ is continuous at all $t \leq \tilde{t}$. By the definition of $\tilde{t}$, there exists some $\delta>0$ such that for all $\epsilon>0$ sufficiently small,

$$
\left|\mathbf{h}(\tilde{t})-G_{\epsilon}\right|>\delta, \quad \forall G_{\epsilon} \in \mathscr{G}(\overline{\mathbf{p}}(\tilde{t}-\epsilon)) .
$$

This contradicts the fact that for all $\tilde{\mathbf{p}}$ and $\tilde{G} \in \mathscr{G}(\tilde{\mathbf{p}})$, $(\mathbf{p}, G(\mathbf{p}))$ is uniquely defined in a neighborhood of $(\tilde{\mathbf{p}}, \tilde{G})$ (cf. Proposition 3 ). Thus, the function $\mathcal{T}_{\mathbf{h}}$ must be empty, implying that $\mathbf{h}$ is a continuous function, as claimed.

Back to the Proof of Theorem 1: Assume, to arrive at a contradiction, that there exist $G^{1}$ and $G^{2}\left(G^{1} \neq G^{2}\right)$ such that $G^{1}, G^{2} \in \mathscr{G}(\mathbf{p})$. By Lemma 2, it follows that there exist continuous functions, $\mathbf{h}_{1}(\cdot)$ and $\mathbf{h}_{2}(\cdot)$, such that $\mathbf{h}_{1}(0)=$ $\mathbf{h}_{2}(0)=G(\hat{\mathbf{p}}) ; \mathbf{h}_{1}(1)=G^{1}$ and $\mathbf{h}_{2}(1)=G^{2}$. Then, there must exist a $\tau=\max \left\{t \in[0,1]: \mathbf{h}_{1}(s)=\mathbf{h}_{2}(s), 0 \leq s \leq t\right\}$. Since we know that $G(\hat{\mathbf{p}})$ is unique, there must be a bifurcation of the $(\overline{\mathbf{p}}(t), G(\overline{\mathbf{p}}(t))$ as $t$ exceeds $\tau$. But, this contradicts the local uniqueness result of Proposition 3. Hence, $\mathscr{G}(\mathbf{p})$ [and therefore $\mathscr{R}(\mathbf{p})]$ has a unique element for all $\mathbf{p} \in(0,1)^{L}$.

Theorem 1 combined with the upper-semicontinuity of Proposition 2 directly implies the continuity of the unique fixed point solution $G(\mathbf{p})$, and hence of $\rho(\mathbf{p})$. This is stated in the following corollary.

Corollary 2. The unique CSMA fixed points $G(\mathbf{p})$ and $\rho(\mathbf{p})$ are both continuous in $\mathbf{p} \in(0,1)^{L}$. 


\section{APPENDIX C \\ PROOF OF THEOREM 3}

Recall that Theorem 3 concerns a sequence of networks for which the number of nodes $N$ increases to infinity. Let $\mathcal{L}^{(N)}$ be the set of all links in the network with $N$ nodes, and let $\mathcal{N}_{i}^{(N)}$ be the set of neighbors of node $i$. Furthermore, let $\left\{\mathbf{p}^{(N)}\right\}_{N \geq 1}$ be a sequence of CSMA policies where $\mathbf{p}^{(N)}$ defines a CSMA policies for the network with $N$ nodes, and let $\left\{\beta^{(N)}\right\}_{N \geq 1}$ be the corresponding sequence of sensing periods. By Assumption 2, the following conditions hold.

(a) For the sequence $\left\{\beta^{(N)}\right\}_{N \geq 1}$ we have

$$
\lim _{N \rightarrow \infty} N \beta^{(N)}=0 \text {. }
$$

(b) For $p_{\max }^{(N)}=\max _{(i, j) \in \mathcal{L}^{(N)}} p_{(i, j)}^{(N)}$ we have that

$$
\lim _{N \rightarrow \infty} \frac{p_{\max }^{(N)}}{\beta^{(N)}}=0 .
$$

(c) There exists a constant $\chi$ and an integer $N_{0}$ such that for all $N \geq N_{0}$ we have that

$$
\sum_{j \in \mathcal{N}_{i}^{(N)}}\left[p_{(i, j)}^{(N)}+p_{(j, i)}^{(N)}\right] \leq \chi \beta^{(N)}, \quad i=1, \ldots, N .
$$

For this setup, Theorem 3 states that

$$
\lim _{N \rightarrow \infty} \delta_{\rho}^{(N)}=0, \quad \text { and } \quad \lim _{N \rightarrow \infty} \delta_{\tau}^{(N)}=0,
$$

where $\delta_{\rho}^{(N)}$ and $\delta_{\rho}^{(N)}$ are as defined in Section VIII.

To prove Theorem 3, we use techniques and results that were presented by Hajek and Krishna in [17] for their analysis of blocking probabilities in loss networks. Before we start the analysis, we provide in the next section a brief summary of [17] as it relates to our analysis. In Section C-B, we provide an overview of the proof.

\section{A. Result by Hajek and Krishna}

Here we provide a brief summary of the work by Hajek and Krishna, we refer to [17] for a more detailed description. Consider a wired (loss) network consisting of a set of undirected links $\mathcal{L}$, where each link $i \in \mathcal{L}$ has capacity 1 . The network serves connections (calls) where each connection uses 1 unit of the capacity at each link it traverses, i.e. when active each link can accommodate at most 1 connection. Furthermore, suppose that all connections use routes that consist of exactly two links. Connection requests arrive according to independent Poisson processes where $\nu_{i j}=\nu_{j i}$ denotes the arrival rate for connections that use links $i$ and $j$. Once a connection is accepted, it stays in the system for an amount of time that is exponentially distributed with mean one. If a new connection that uses links $i$ and $j$ in its route arrives and one of these links is already serving another connection, then it is blocked and lost. Then, $B^{E}(\nu)=\left(B_{i}^{E}(\nu)\right)_{i}$ is defined as the solution of the following Erlang fixed point equation

$$
\frac{B_{i}^{E}}{1-B_{i}^{E}}=\sum_{j \in \mathcal{L}} \nu_{i j}\left(1-B_{j}^{E}\right), \quad i \in \mathcal{N}
$$

where $B_{i}^{E}$ approximates the probability that link $i$ is busy, i.e. the probability of serving an incoming connection. In [17], Hajek and Krishna obtain the following result:

Proposition 4. Consider a loss network as defined above and let

$$
r_{v}=\max _{i, j \in \mathcal{L}} \nu_{i j}
$$

and

$$
\chi=\max _{i \in \mathcal{L}} \sum_{j \in \mathcal{L}} \nu_{i j} .
$$

Then, the actual steady-state probability $\bar{B}_{i}, i \in \mathcal{L}$, that link $i$ is busy satisfies, for all $i \in \mathcal{N}$,

$$
\left(1-B_{i}^{E}\right) e^{-\chi\left(r_{v}+r_{v}^{2} / 2\right)} \leq 1-\bar{B}_{i} \leq\left(1-B_{i}^{E}\right) e^{\chi\left(r_{v}+r_{v}^{2} / 2\right)},
$$

where $B_{i}^{E}, i \in \mathcal{L}$, is the solution to the Erlang fixed point equation given by (26).

The above proposition implies that for small $\chi$ and $r_{v}$, the solution to the Erlang fixed point equation approximates well the actual steady-state probability of a link being busy. Our analysis follows a similar argument whereby we show that our CSMA fixed point equation can be closely approximated by an Erlang fixed point equation, which, in turn, is an accurate estimate of the actual performance of the CSMA policy in the asymptotic regime of large networks and small sensing time. Next section outlines the steps of this argument more explicitly.

\section{B. Main Steps in the Proof of Theorem 3}

In this section, we list the main steps leading to the proof of Theorem 3, and then provide the proof based on those results. The proof of the statements of the steps are moved to subsequent subsections to avoid disruption of the flow.

Step 1) Recall that we previously defined and studied the equations (9)-(10) as fixed point equations with respect to the parameters $\rho(\mathbf{p})$ or $G(\mathbf{p})$. For this proof, we find it move convenient to work with a new parameter $B=\left(B_{1}, \cdots, B_{N}\right)$, where $B_{i} \triangleq\left(1-\rho_{i}\right)$ for each node $i$ that approximates the fraction of busy time of that node under CSMA policy $\mathbf{p}$. To that end, in Section C-C, we let $\nu=\left(\nu_{i j}\right)$ with $\nu_{i j} \triangleq$ $\frac{p_{(i, j)}+p_{(j, i)}}{\beta}$, and define $B(\nu)$ as the solution to the CSMA fixed point equation:

$B_{i}=\frac{\left(1-B_{i}\right)}{\beta}\left(1-\exp \left(-\beta \sum_{j \in \mathcal{N}_{i}} \nu_{i j}\left(1-B_{j}\right)\right)\right), \quad i \in \mathcal{N}$.

Then, in Lemma 4, we relate this CSMA fixed point equation (27) to the following generalized version of the Erlang fixed point equation (26) where $B^{G E}(\nu)$ solves

$$
\frac{B_{i}^{G E}}{1-B_{i}^{G E}}=\sum_{j \in \mathcal{N}_{i}} \nu_{i j}\left(1-B_{j}^{G E}\right), \quad i \in \mathcal{N},
$$

where (in contrast to the Erlang fixed point equation) it is not required that $\nu_{i j}=\nu_{j i}$, but it is allowed

$$
\nu_{i j} \neq \nu_{j i}
$$

Using the generalized Erlang fixed point equation, it is shown that there exists a nonnegative vector $\hat{\nu}=\left(\hat{\nu}_{i j}\right)$ close to $\nu$, 
potentially, with $\hat{\nu}_{i j} \neq \hat{\nu}_{j i}$ that satisfies $B(\nu)=B^{G E}(\hat{\nu})$. Further, we provide bounds on the proximity of $\hat{\nu}$ values to $\nu$ (see Lemma 4 for details).

With this motivation, in Section C-D, we prove the existence and uniqueness of the generalized Erlang fixed point $B^{G E}(\hat{\nu})$ for any nonnegative $\hat{\nu}$, potentially, with $\hat{\nu}_{i j} \neq \hat{\nu}_{j i}$. Then, in Section C-E, we provide a sensitivity analysis of the fixed point $B^{G E}(\hat{\nu})$ to bound the change in the fixed point solution when $\hat{\nu}$ is locally perturbed.

Using this analysis we obtain Corollary 3 which allows to tightly bounds the CSMA fixed point solution $B(\nu)$ with the Erlang fixed point solution $B^{E}(\hat{\nu})$, i.e. Corollary 3 states that the CSMA fixed point $B(\nu)$ and the Erlang fixed point $B^{E}(\nu)$ become (asymptotically) identical for large $N$. The generalized Erlang fixed point $B^{G E}(\hat{\nu})$ serves in this step as a vehicle to related that CSMA fixed point to the Erlang fixed point.

Step 2) In this step, we study the characteristics of the actual asynchronous CSMA policy performance. To that end, in Section C-F, we first prove that the asynchronous CSMA policy has well-defined steady-state distribution, and hence falls within the set $\mathcal{P}$ of policies with well-defined link service rates $\mu=\left(\mu_{i j}\right)$ and probabilities of links being idle $\sigma=\left(\sigma_{i}\right)_{i \in \mathcal{N}}$. Then, in Section C-G, we derive several properties of $\sigma$ which are then used in Section $\mathrm{C}-\mathrm{H}$ to prove that the steady-state probabilities of nodes being idle become asymptotically independent in the large network and small sensing delay limit.

Step 3) Combining the results from Steps 1 and 2, we show in Section C-I that under Assumption 2 the solution to the CSMA fixed point equation is asymptotically accurate. In particular, we derive the following important result (see Section C-I for its proof).

Proposition 5. Consider a CSMA policy $\left(\mathbf{p}^{(N)}, \beta^{(N)}\right)$ for a wireless network consisting of $N$ nodes and let $p_{\max }^{(N)}=$ $\max _{(i, j) \in \mathcal{L}} p_{(i, j)}^{(N)}$ and let $\chi$ be as defined in Assumption 2(c).

Then, there exist constants positive $\kappa$ and $\kappa_{s}$ that do not depend on $N$, and an integer $N_{0}$, such that for $N>N_{0}$ the actual steady-state probability $\sigma_{i}\left(\mathbf{p}^{(N)}\right), i \in \mathcal{N}$, that node $i$ is idle under the CSMA policy $\left(\mathbf{p}^{(N)}, \beta^{(N)}\right)$ satisfy, $\forall i \in \mathcal{N}$,

$$
\begin{aligned}
& \rho_{i}\left(\mathbf{p}^{(N)}\right) e^{-\chi\left(r+r^{2} / 2\right)} e^{-\chi\left(\kappa \beta^{(N)}+\left(\kappa \beta^{(N)}\right)^{2} / 2\right)} \\
& \quad \leq \sigma_{i}\left(\mathbf{p}^{(N)}\right) \leq \rho_{i}\left(\mathbf{p}^{(N)}\right) e^{\chi\left(r+r^{2} / 2\right)} e^{\chi\left(\kappa \beta^{(N)}+\left(\kappa \beta^{(N)}\right)^{2} / 2\right)},
\end{aligned}
$$

where $\rho_{i}\left(\mathbf{p}^{(N)}\right)$ is the solution to the CSMA fixed point equation for $\mathbf{p}^{(N)}$, and

$$
r \triangleq 2\left[(2 N+1)\left(\kappa_{s} \beta^{(N)}\right)+2 r_{p}\right], \quad \text { with } \quad r_{p} \triangleq \frac{p_{m a x}^{(N)}}{\beta^{(N)}} .
$$

Based on Steps 1-3 and Proposition 5, we can now prove Theorem 3.

Proof of Theorem 3: Consider a sequence of CSMA policies $\mathbf{p}^{(N)}$ that satisfies Assumption 2. To keep the notation light, we use in the following only $\mathbf{p}$ instead of $\mathbf{p}^{(N)}, p_{i j}$ instead of $p_{(i, j)}^{(N)}, \sigma_{i}$ instead of $\sigma_{i}\left(\mathbf{p}^{(N)}\right), \rho_{i}$ instead of $\rho_{i}\left(\mathbf{p}^{(N)}\right)$, and $\beta$ instead of $\beta^{(N)}$. Furthermore, we use $\mu_{(i, j)}$ instead of $\mu_{(i, j)}\left(\mathbf{p}^{(N)}\right)$ to denote the link service rate for link $(i, j)$ under the CSMA policy $\mathbf{p}^{(N)}$, and $\tau_{(i, j)}$ instead of $\tau_{(i, j)}\left(\mathbf{p}^{(N)}\right)$ to denote the approximation of link service rate for link $(i, j)$ under the CSMA fixed point approximation for the CSMA policy $\mathbf{p}^{(N)}$.

We first show that

$$
\lim _{N \rightarrow \infty} \delta_{\rho}^{(N)}=0 .
$$

This result follows immediately from Proposition 5 which states that the steady-state probabilities asymptotically converge to the solution of the CSMA fixed point equation if

$$
\lim _{N \rightarrow \infty}\left((2 N+1)\left(\kappa_{s} \beta\right)+2 r_{p}\right)=0,
$$

or

$$
\lim _{N \rightarrow \infty} N \beta=0, \quad \text { and } \quad \lim _{N \rightarrow \infty} \frac{p_{\max }^{(N)}}{\beta}=0 .
$$

And indeed, these conditions hold by Assumption 2 .

The proof that

$$
\lim _{N \rightarrow \infty} \delta_{\tau}^{(N)}=0
$$

requires results that we obtain in Section $\mathrm{C}-\mathrm{G}$ and $\mathrm{C}-\mathrm{H}$ (outlined in Step 2 above); we will provide references to these results in the derivations below.

We are going to use the following convention. We say that a node $i$ is idle if node $i$ is currently neither sending, nor receiving, a data packet. Otherwise, we say that node $i$ is busy. Accordingly, we say that a link $l=(i, j)$ is idle if both node $i$ and $j$ are idle. Otherwise, we say that link $(i, j)$ is busy.

Let $y_{i}$ be the indicator whether node $i$ is idle $\left(y_{i}=0\right)$ or busy $\left(y_{i}=1\right)$, and let $P\left(y_{i}=0, y_{j}=0\right)$ be the steady-state probabilities that node $i$ and $j$ are jointly idle. In Section C-F, we show this steady-state probability exists. Then, using the same argument as we give in Section C-G to prove Lemma 20, we can see that

$$
\begin{aligned}
P\left(y_{i}=0, y_{j}=0\right) p_{(i, j)}(1-4 \chi \beta)^{2} & \\
& \leq \mu_{(i, j)} \beta \leq \frac{P\left(y_{i}=0, y_{j}=0\right) p_{(i, j)}}{(1-4 \chi \beta)},
\end{aligned}
$$

where $(1-4 \chi \beta)$ is a lower-bound (see Section C-G) on the probability that a packet transmission on link $(i, j)$ is successful, i.e. does not experience a collision.

Also, by Proposition 8 in Section $\mathrm{C}-\mathrm{H}$, we have that

$$
\begin{aligned}
\frac{1}{1+2 r_{p}}\left(\frac{1}{1+\kappa_{s} \beta}\right)^{2 N} & \leq \frac{P\left(y_{i}=0, y_{j}=0\right)}{\sigma_{i} \sigma_{j}} \\
& \leq\left(1+\kappa_{s} \beta\right)^{2 N}\left(1+2 r_{p}\right) .
\end{aligned}
$$

Using this result in the previous expression yields

$$
\begin{aligned}
& \frac{1}{1+2 r_{p}}\left(\frac{1}{1+\kappa_{s} \beta}\right)^{2 N} \sigma_{i} \sigma_{j} p_{(i, j)}(1-4 \chi \beta)^{2} \\
& \quad \leq \mu_{(i, j)} \beta \leq\left(1+\kappa_{s} \beta\right)^{2 N}\left(1+2 r_{p}\right) \sigma_{i} \sigma_{j} p_{(i, j)} \frac{1}{1-4 \chi \beta} .
\end{aligned}
$$

Combining this result with Proposition 5, we obtain that

$$
\begin{aligned}
& \left(\frac{1}{1+\kappa_{s} \beta}\right)^{2 N} \frac{(1-4 \chi \beta)^{2}}{\left(1+2 r_{p}\right)} e^{-2 \chi\left(r+\frac{r^{2}}{2}\right)} e^{-2 \chi\left(\kappa \beta+\frac{(\kappa \beta)^{2}}{2}\right)} \\
& \quad \leq \frac{\mu_{(i, j)} \beta}{\rho_{i} \rho_{j} p_{(i, j)}} \\
& \quad \leq\left(1+\kappa_{s} \beta\right)^{2 N} \frac{\left(1+2 r_{p}\right)}{(1-4 \chi \beta)} e^{2 \chi\left(r+\frac{r^{2}}{2}\right)} e^{2 \chi\left(\kappa \beta+\frac{(\kappa \beta)^{2}}{2}\right)},
\end{aligned}
$$


where $\rho_{i}$ and $\rho_{j}$ are the solutions to the CSMA fixed point equation (9)-(10) for the CSMA policy $\mathbf{p}$.

As we have that (see Sections VI-B and VII, and Equations (11) and (12))

or

$$
\begin{aligned}
\frac{\rho_{j} p_{(i, j)} e^{-2 \chi \beta}}{1+\beta-e^{-G_{i}(\mathbf{p})}} & \leq \frac{\rho_{j} p_{(i, j)} e^{-\left(G_{i}(\mathbf{p})+G_{j}(\mathbf{p})\right)}}{1+\beta-e^{-G_{i}(\mathbf{p})}} \\
& \leq \tau_{(i, j)} \leq \frac{\rho_{j} p_{(i, j)}}{1+\beta-e^{-G_{i}(\mathbf{p})}}
\end{aligned}
$$

$$
\frac{\rho_{i} \rho_{j} p_{(i, j)} e^{-2 \chi \beta}}{\beta} \leq \tau_{(i, j)} \leq \frac{\rho_{i} \rho_{j} p_{(i, j)}}{\beta},
$$

it follows that

$$
\begin{aligned}
& \left(\frac{1}{1+\kappa_{s} \beta}\right)^{2 N} \frac{(1-4 \chi \beta)}{\left(1+2 r_{p}\right)} e^{-2 \chi\left(r+\frac{r^{2}}{2}\right)} e^{-2 \chi\left(\kappa \beta+\frac{(\kappa \beta)^{2}}{2}\right)} e^{-2 \chi \beta} \\
& \leq \frac{\tau_{(i, j)}}{\mu_{(i, j)}} \\
& \leq\left(1+\kappa_{s} \beta\right)^{2 N} \frac{\left(1+2 r_{p}\right)}{(1-4 \chi \beta)^{2}} e^{2 \chi\left(r+\frac{r^{2}}{2}\right)} e^{2 \chi\left(\kappa \beta+\frac{(\kappa \beta)^{2}}{2}\right)}
\end{aligned}
$$

Finally, note that under Assumption 2, we have

$$
\lim _{N \rightarrow \infty} \frac{(1-4 \chi \beta) e^{-2 \chi\left(r+\frac{r^{2}}{2}\right)} e^{-2 \chi\left(\kappa \beta+\frac{(\kappa \beta)^{2}}{2}\right)} e^{-2 \chi \beta}}{\left(1+2 r_{p}\right)\left(1+\kappa_{s} \beta\right)^{2 N}}=1
$$

and

$$
\lim _{N \rightarrow \infty} \frac{\left(1+\kappa_{s} \beta\right)^{2 N}\left(1+2 r_{p}\right) e^{2 \chi\left(r+\frac{r^{2}}{2}\right)} e^{2 \chi\left(\kappa \beta+\frac{(\kappa \beta)^{2}}{2}\right)}}{(1-4 \chi \beta)}=1 .
$$

Therefore, it follows that $\lim _{N \rightarrow \infty} \delta_{\tau}^{(N)}=0$.

\section{Alternative Formulation of the CSMA Fixed Point}

In this section, we derive an alternative formulation for the CSMA fixed point for a CSMA policy $\mathbf{p}$, which is then used to relate the CSMA fixed point to the Erlang fixed point for loss networks (as outlined in Step 1 of Section C-B). To keep the notation light, we use in the following $p_{(i, j)}$ instead of $p_{(i, j}^{(N)}, \beta$ instead of $\beta^{(N)}, G_{i}$ instead of $G_{i}^{(N)}$, and $\mathcal{N}_{i}$ instead of $\mathcal{N}_{i}^{(N)}$.

Recall that for a CSMA policy $\mathbf{p}$ with sensing period $\beta$, the CSMA fixed point equation is given by

$$
\rho_{i}=\frac{\beta}{\beta+1-e^{-G_{i}}}, \quad i=1, \ldots, N,
$$

where $G_{i}=\sum_{j \in \mathcal{N}_{i}}\left(p_{(i, j)}+p_{(j, i)}\right) \rho_{j}$. First we observe that for large $N$ the offered load $G_{i}$ becomes small at all nodes.

Lemma 3. Under Assumption 2, we have

$$
\lim _{N \rightarrow \infty} G_{i}=0, \quad i=1, \ldots, N .
$$

Proof: By Assumption 2, we have that

$$
\begin{aligned}
\lim _{N \rightarrow \infty} G_{i} & =\lim _{N \rightarrow \infty} \sum_{j \in \mathcal{N}_{i}}\left(p_{(i, j)}+p_{(j, i)}\right) \rho_{j} \\
& \leq \lim _{N \rightarrow \infty} \sum_{j \in \mathcal{N}_{i}}\left(p_{(i, j)}+p_{(j, i)}\right) \\
& \leq \lim _{N \rightarrow \infty} \chi \beta \\
& =0 .
\end{aligned}
$$

Let $B(\nu)=\left(B_{i}(\nu)\right)_{i}$ be the CSMA fixed point as given by equation (27), i.e. $B(\nu)$ is the solution to the fixed point equation

$$
B_{i}=1-\frac{\beta}{\beta+1-e^{-G_{i}}}, \quad i=1, \ldots, N,
$$

where $G_{i}=\sum_{j \in \mathcal{N}_{i}}\left(p_{(i, j)}+p_{(j, i)}\right)\left(1-B_{j}\right)$. Note that we can rewrite the expression for $B_{i}$ as

$$
\begin{aligned}
B_{i} & =\frac{\beta}{\beta+1-e^{-G_{i}}} \frac{1}{\beta}\left(1-e^{-G_{i}}\right)=\rho_{i} \frac{1}{\beta}\left(1-e^{-G_{i}}\right) \\
& =\left(1-B_{i}\right) \frac{1}{\beta}\left(1-e^{-G_{i}}\right),
\end{aligned}
$$

which is previously posed as (27) in our outline. We then have the following result.

Lemma 4. Given a CSMA policy $(\mathbf{p}, \beta)$ for a network with $N$ nodes, let

$$
\nu_{i j}=\frac{p_{(i, j)}+p_{(j, i)}}{\beta}, \quad i, j=1, \ldots, N
$$

and let $\chi$ be given as in Assumption 2(c). Let $B(\nu), i=$ $1, \ldots, N$, be the CSMA fixed point as given by (27). Then, for $\beta \in\left[0,(2 \chi)^{-1}\right]$ and $\kappa \geq 2 \chi$ we have that

$$
B_{i}(\nu)=\left(1-B_{i}(\nu)\right) \sum_{j \in \mathcal{N}_{i}} \hat{\nu}_{i j}\left(1-B_{j}(\nu)\right), \quad i \in \mathcal{N},
$$

for some $\hat{\nu}_{i j} \geq 0$, potentially, with $\hat{\nu}_{i j} \neq \hat{\nu}_{j i}$ satisfying

$$
\frac{1}{1+\kappa \beta} \leq \frac{\hat{\nu}_{i j}}{\nu_{i j}} \leq 1+\kappa \beta, \quad(i, j) \in \mathcal{L},
$$

and $\hat{\nu}_{i, j}=0$ if $(i, j) \notin \mathcal{L}$. More compactly, we have $B(\hat{\nu})=$ $B^{G E}(\nu)$ where $B^{G E}(\hat{\nu})$ is defined in equation (28).

Proof: For

$$
G_{i}=\sum_{j \in \mathcal{N}_{i}}\left(p_{(i, j)}+p_{(j, i)}\right)\left(1-B_{j}(\nu)\right)
$$

note that

$$
1-e^{-G_{i}} \leq G_{i} \leq G_{i}(1+\kappa \beta) \text {. }
$$

Furthermore, we have

$$
G_{i} e^{-G_{i}} \leq 1-e^{-G_{i}} .
$$

To see this, note that $\lim _{x \rightarrow 0} x e^{-x}=\lim _{x \rightarrow 0}\left(1-e^{-x}\right)=0$ and $\frac{d}{d x} x e^{-x} \leq \frac{d}{d x}\left(1-e^{-x}\right)$, for $x \geq 0$. Since, by Assumption 2, we have $G_{i} \leq \chi \beta$, it also follows that

$$
G_{i} e^{-\chi \beta} \leq G_{i} e^{-G_{i}} \leq 1-e^{-G_{i}} .
$$

Furthermore, since $e^{-\chi \beta} \geq(1-\chi \beta)$, it follows that

$$
G_{i}(1-\chi \beta) \leq 1-e^{-G_{i}}
$$

Finally, for $\kappa \geq \frac{\chi}{1-\chi \beta}$, we have

$$
\frac{1}{1+\kappa \beta} \leq \frac{1}{1+\frac{\chi}{1-\chi \beta} \beta}=1-\chi \beta \text {. }
$$


Combining the above results, it then follows that for $\beta \in$ $\left[0,(2 \chi)^{-1}\right]$ and $\kappa \geq 2 \chi$ we have

$$
G_{i} \frac{1}{1+\kappa \beta} \leq 1-e^{-G_{i}} \leq G_{i}(1+\kappa \beta)
$$

and, using (29),

$$
\begin{aligned}
& \frac{1}{1+\kappa \beta} \sum_{j \in \mathcal{N}_{i}} \nu_{i j}\left(1-B_{i}(\nu)\right)\left(1-B_{j}(\nu)\right) \\
& \leq \quad B_{i}(\nu) \leq \\
& \quad(1+\kappa \beta) \sum_{j \in \mathcal{N}_{i}} \nu_{i j}\left(1-B_{i}(\nu)\right)\left(1-B_{j}(\nu)\right), \quad \forall i \in \mathcal{N} .
\end{aligned}
$$

The result then immediately follows from continuity and the fact that for node $i \in \mathcal{N}$ the vector $\left(\hat{\nu}_{i j}\right)_{j \in \mathcal{N}_{i}}$, can be determined independently from the other nodes.

Thus, the above lemma establishes that the CSMA fixed point $B(\nu)$ that solves (27) can alternatively be expressed as the fixed point that solves the generalized Erlang fixed point equation (28) where the true transmission rates $\nu_{i j}$ are replaced by "approximate transmission rates" $\hat{\nu}_{i j}$.

\section{Existence and Uniqueness of a Fixed Point}

Consider the generalized Erlang fixed point equation of Lemma 4 that is given by

$$
B_{i}^{G E}=\sum_{j \in \mathcal{N}_{i}} \hat{\nu}_{i j}\left(1-B_{i}^{G E}\right)\left(1-B_{j}^{G E}\right), \quad i \in \mathcal{N},
$$

with

$$
\hat{\nu}_{i j} \geq 0, \quad(i, j) \in \mathcal{L},
$$

where we allow that

$$
\hat{\nu}_{i j} \neq \hat{\nu}_{j i}
$$

In this section, we will show that there exists a unique fixed point by using an argument that is similar to the one in Section B that we used to prove the existence and uniqueness of the CSMA fixed point.

We first rewrite the above fixed point equation as

$$
\frac{B_{i}^{G E}}{1-B_{i}^{G E}}=\sum_{j \in \mathcal{N}_{i}} \hat{\nu}_{i j}\left(1-B_{j}^{G E}\right), \quad i \in \mathcal{N}
$$

where $\hat{\nu}_{i j} \geq 0,(i, j) \in \mathcal{L}$.

Given vector $\hat{\nu}=\left(\hat{\nu}_{i j}\right)_{(i, j) \in \mathcal{L}}$ with $\hat{\nu}_{i j} \geq 0,(i, j) \in \mathcal{L}$, let $B^{G E}(\hat{\nu})$ be the set of fixed points for Eq. (30). Then we have the following result.

Lemma 5. For all fixed points $B^{\bar{G} E} \in B^{G E}(\hat{\nu})$, there exist neighborhoods $U \subset \mathbb{R}_{+}^{N}$ of $B^{\bar{G} E}$ and $V \subset[0,1]^{L}$ of $\hat{\nu}$ such that for each $\nu \in V$ the equation $F\left(B^{G E}, \nu\right)=$ $\left(F_{i}\left(B^{G E}, \nu\right)\right)_{i \in \mathcal{N}}=0$ where

$$
F_{i}\left(B^{G E}, \nu\right)=\frac{B_{i}^{G E}}{1-B_{i}^{G E}}-\sum_{j \in \mathcal{N}_{i}} \nu_{i j}\left(1-B_{j}^{G E}\right)
$$

has a unique solution $B^{G E} \in U$. Moreover, this solution can be given by a function $B^{G E}=\phi(\nu)$ where $\phi$ is continuously differentiable on $V$.
Proof: For $i \in \mathcal{N}$, we have

$$
\frac{\partial F_{i}}{\partial B_{j}^{G E}}= \begin{cases}\frac{1}{\left(1-B_{i}^{G E}\right)^{2}}, & i=j \\ \nu_{i j}, & j \in \mathcal{N}_{i} \\ 0, & \text { otherwise. }\end{cases}
$$

Note that the function $F$ is continuously differentiable. Next we show that the Jacobain matrix

$$
\left[\frac{\partial F_{i}}{\partial B_{j}^{G E}}\right]
$$

has linearly independent rows. Having established this result, the lemma then follows from the implicit function theorem. Before we proceed, we note that this matrix has non-negative entries.

Suppose that the rows are not linearly independent, then there exists a coefficient vector $\mathbf{x}=\left(x_{1}, \ldots, x_{N}\right) \neq \mathbf{0}$ such that

$$
\sum_{j=1}^{N} x_{j}\left(\frac{\partial F_{i}\left(B^{G E}, \nu\right)}{\partial B_{j}^{G E}}\right)=0, \quad \text { for all } i \in\{1, \ldots, N\} .
$$

Using the special structure of the Jacobian matrix, we obtain

$$
\frac{x_{i}}{\left(1-B_{i}^{G E}\right)^{2}}+\sum_{j \in \mathcal{N}_{i}} x_{j} \nu_{i j}=0, \quad i \in \mathcal{N},
$$

or

$$
1+\sum_{j \in \mathcal{N}_{i}} \nu_{i j} \frac{x_{j}}{x_{i}}\left(1-B_{i}^{G E}\right)^{2}=0, \quad i \in \mathcal{N} .
$$

Consider a node $i^{*}$ such that

$$
\left|\frac{x_{i^{*}}}{1-B_{i^{*}}^{G E}}\right| \geq\left|\frac{x_{i}}{1-B_{i}^{G E}}\right|, \quad i \in \mathcal{N} .
$$

Then,

$$
\begin{aligned}
1 & =-\sum_{j \in \mathcal{N}_{i^{*}}} \nu_{i^{*} j}\left(1-B_{i^{*}}^{G E}\right)\left(1-B_{j}^{G E}\right) \frac{x_{j}}{x_{i^{*}}} \frac{1-B_{i^{*}}^{G E}}{1-B_{j}^{G E}} \\
& \leq \sum_{j \in \mathcal{N}_{i^{*}}} \nu_{i^{*} j}\left(1-B_{i^{*}}^{G E}\right)\left(1-B_{j}^{G E}\right)\left|\frac{x_{j}}{x_{i^{*}}} \frac{1-B_{i^{*}}^{G E}}{1-B_{j}^{G E}}\right| \\
& \leq \sum_{j \in \mathcal{N}_{i^{*}}} \nu_{i^{*} j}\left(1-B_{i^{*}}^{G E}\right)\left(1-B_{j}^{G E}\right)=B_{i^{*}}^{G E}<1 .
\end{aligned}
$$

Hence, we obtain a contradiction and the result follows.

We then obtain the following result by the same argument as given to prove the uniqueness of the CSMA fixed point in Section B.

Lemma 6. There exists a unique fixed point $B^{G E}(\hat{\nu})$ to Eq. (30).

\section{E. Sensitivity Analysis}

In this section we show that asymptotically (as $N$ becomes large) the solution to the CSMA fixed point equation converges to the solution of the Erlang fixed point equation given by Eq. (26). To show this, we use a sensitivity analysis for the generalized Erlang fixed point $B^{G E}(\hat{\nu})$ that is the same as given by Hajek and Krishna in Section 4 of [17] with only minor notational changes. For convenience, we provide below 
the analysis of Hajek and Krishna applied to the generalized Erlang fixed point $B^{G E}(\hat{\nu})$.

Given vector $\nu=\left(\nu_{i j}\right)_{(i, j) \in \mathcal{L}}$ with $\nu_{i j} \geq 0,(i, j) \in \mathcal{L}$, let $B^{G E}(\nu)=\left(B_{1}^{G E}(\nu), \ldots, B_{N}^{G E}(\nu)\right)$ be the fixed point to the equation

$$
\frac{B_{i}^{G E}}{1-B_{i}^{G E}}=\sum_{j \in \mathcal{N}_{i}} \nu_{i j}\left(1-B_{j}^{G E}\right), \quad i \in \mathcal{N},
$$

where we allow that $\nu_{i j} \neq \nu_{j i}$. Furthermore, let the links $l=(i, j) \in \mathcal{L}$ be indexed with numbers $1, \ldots, L$.

Consider then $F\left(B^{G E}, v\right)$

$\left(F_{1}\left(B^{G E}, v\right), \ldots, F_{N}\left(B^{G E}, v\right)\right) \quad$ where the function $F_{i}\left(B^{G E}, v\right)$ is given by

$F_{i}\left(B^{G E}, \nu\right)=\frac{B_{i}^{G E}}{1-B_{i}^{G E}}-\sum_{j \in \mathcal{N}_{i}} \nu_{i j}\left(1-B_{j}^{G E}\right), \quad i \in \mathcal{N}$.

with $\nu_{i j} \geq 0,(i, j) \in \mathcal{L}$.

We then have

$$
\frac{\partial F_{i}}{\partial B_{j}^{G E}}=\frac{1}{\left(1-B_{i}^{G E}\right)^{2}} I_{j=i}+\nu_{i j}, \quad i, j \in \mathcal{N},
$$

and

$$
\frac{\partial F_{i}}{\partial \nu_{i j}}=-\left(1-B_{j}^{G E}\right), \quad i \in \mathcal{L}, j \in \mathcal{N}_{i} .
$$

Let $\mathbb{D}$ be the $N \times N$ diagonal matrix with

$$
\mathbb{D}_{i, i}=\left(1-B_{i}^{G E}\right) .
$$

Furthermore, let $\mathbb{R}$ be the $N \times N$ matrix given by

$$
\mathbb{R}_{i, j}= \begin{cases}\nu_{i j}, & j \in \mathcal{N}_{i}, \\ 0, & \text { otherwise },\end{cases}
$$

and let $\mathbb{T}$ be the $N \times|\mathcal{L}|$ matrix given by

$$
\mathbb{T}_{i, l}= \begin{cases}\left(1-B_{i}^{G E}\right)\left(1-B_{j}^{G E}\right), & l=(i, j), j \in \mathcal{N}_{i}, \\ 0, & \text { otherwise. }\end{cases}
$$

Using the above definitions, we then have that

$$
\frac{\partial F}{\partial B^{G E}}=\mathbb{D}^{-2}+\mathbb{R}
$$

and

$$
\frac{\partial F}{\partial v}=-\mathbb{D}^{-1} \mathbb{T}
$$

Finally, let

$$
\mathbb{L}=(\mathbb{I}+\mathbb{D} \mathbb{R D})^{-1}
$$

where II is the identity matrix. Then we have the following result.

Lemma 7. The matrix $\mathbb{L}$ is well-defined and

$$
\sum_{j \in \mathcal{N}}\left|\mathbb{L}_{i, j}\right| \leq \frac{1}{1-B_{*}^{G E}}, \quad i \in \mathcal{N},
$$

where $B_{*}^{G E}=\max _{i \in \mathcal{N}} B_{i}^{G E}$.

Proof: Recall that

$$
\frac{\partial F}{\partial B^{G E}}=\mathbb{D}^{-2}+\mathbb{R}
$$

which we can rewrite as

$$
\frac{\partial F}{\partial B^{G E}}=\mathbb{D}^{-1}(\mathbb{I}+\mathbb{D} \mathbb{R D}) \mathbb{D}^{-1} .
$$

By Lemma 5, the matrix $\frac{\partial F}{\partial B^{G E}}$ is invertible. It follows that $(\mathbb{I}+\mathbb{D R D})$ is invertible and $\mathbb{L}$ is well defined.

To show that

$$
\sum_{j \in \mathcal{N}}\left|\mathbb{L}_{i, j}\right| \leq \frac{1}{1-B_{*}^{G E}}, \quad i \in \mathcal{N},
$$

we can use the same argument as given to prove Lemma 1 in [17]. That is, let $\mathbb{M}=\mathbb{D R D}$, so the diagonal elements of $\mathbb{M}_{i, i}$ are all equal to zero and the off-diagonal elements are given by

$$
\mathbb{M}_{i, j}=\left(1-B_{i}^{G E}\right)\left(1-B_{j}^{G E}\right) \nu_{i j} .
$$

Note that the elements of $\mathbb{M}$ are all non-negative and that

$$
\sum_{j \in \mathcal{N}} \mathbb{M}_{i, j}=\left(1-B_{i}^{G E}\right) \sum_{j \in \mathcal{N}_{i}} \nu_{i j}\left(1-B_{j}^{G E}\right)=B_{i}^{G E} .
$$

Let $e$ denote the vector with all elements being equal to 1 . Then we have that

$$
\mathbb{M} e \leq B_{*}^{G E},
$$

where the inequality is understood to be coordinate-bycoordinate. By induction, we obtain for $n \geq 0$ that

$$
\mathbb{M}^{n} e \leq\left(B_{*}^{G E}\right)^{n}
$$

and $\mathbb{L}$ is given by the absolute convergent series

$$
\mathbb{L}=\sum_{n=0}^{\infty}(-1)^{n} \mathbb{M}^{n} \text {. }
$$

Moreover, for the matrix $|\mathbb{L}|$ given by

$$
|\mathbb{L}|_{i, j}=\left|\mathbb{L}_{i, j}\right|
$$

we have

$$
|\mathbb{L}| e \leq \sum_{n=0}^{\infty} \mathbb{M}^{n} e \leq \sum_{n=0}^{\infty}\left(B_{*}^{G E}\right)^{n} e=\frac{1}{1-B_{*}^{G E}} e,
$$

and the lemma follows.

From the proof of Lemma 7, we have that

$$
\frac{\partial B^{G E}}{\partial \nu}=\left(\mathbb{D}^{-2}+\mathbb{R}\right)^{-1} \mathbb{D}^{-1} \mathbb{T}=\mathbb{D L} \mathbb{T} .
$$

We use this result as follows. Let $B^{G E}(s)$ be the solution to the fixed point equation

$$
\frac{B_{i}^{G E}}{1-B_{i}^{G E}}=\sum_{j \in \mathcal{N}_{i}} \hat{\nu}_{i j}(s)\left(1-B_{j}^{G E}\right), \quad i \in \mathcal{N},
$$

with

$$
\hat{\nu}_{i j}(s)=\nu_{i j}\left(1+\delta_{i j} s\right), \quad-\frac{1}{(1+\kappa \beta)} \leq \delta_{i j} \leq 1 .
$$

Note that as we vary $\delta_{i j}$ in the interval $\left[-\frac{1}{(1+\kappa \beta)}, 1\right]$ and $s$ in the interval $[0, \kappa \beta], \hat{\nu}_{i j}$ will vary in the interval $\left[\frac{1}{(1+\kappa \beta)}, 1+\right.$ $\kappa \beta]$.

Using the fact that

$$
\frac{\partial B^{G E}}{\partial \hat{\nu}}=\mathbb{D} \mathbb{L} \mathbb{T}
$$


and the chain rule

$$
\frac{d B_{k}^{G E}}{d s}=\frac{\partial B_{k}^{G E}}{\partial \hat{\nu}} \frac{d \hat{\nu}}{d s}
$$

we obtain for $B_{k}^{G E}(s), k=1, . ., N$, that

$$
\begin{aligned}
&\left|\frac{d B_{k}^{G E}}{d s}\right|=\left|\sum_{i \in \mathcal{N}} \sum_{j \in \mathcal{N}_{i}} \frac{d B_{k}^{G E}}{d \hat{\nu}_{i j}} \nu_{i j} \delta_{i j}\right| \\
& \leq \sum_{i \in \mathcal{N}} \sum_{j \in \mathcal{N}_{i}}\left|\frac{d B_{k}^{G E}}{d \hat{\nu}_{i j}} \nu_{i j} \delta_{i j}\right| \\
& \leq\left(1-B_{k}^{G E}\right) \sum_{i \in \mathcal{N}}\left|\mathbb{L}_{k, i}\right| \sum_{j \in \mathcal{N}_{i}} \mathbb{T}_{i,(i, j)}\left|\nu_{i j} \delta_{j i}\right| \\
&=\left(1-B_{k}^{G E}\right) \sum_{i \in \mathcal{N}}\left\{\left|\mathbb{L}_{k, i}\right| \cdot\right. \\
&\left.\sum_{j \in \mathcal{N}_{i}}\left(1-B_{i}^{G E}\right)\left(1-B_{j}^{G E}\right) \nu_{i j}\left|\delta_{i j}\right|\right\}
\end{aligned}
$$

As we have that $\nu_{i j}=0$ for $j \notin \mathcal{N}_{i}$, we obtain that

$$
\begin{aligned}
&\left|\frac{d B_{k}^{G E}}{d s}\right| \leq\left(1-B_{k}^{G E}\right) \sum_{i \in \mathcal{N}}\left\{\left|\mathbb{L}_{k, i}\right| \cdot\right.\left.\sum_{j \in \mathcal{N}_{i}}\left(1-B_{i}^{G E}\right)\left(1-B_{j}^{G E}\right) \nu_{i j}\left|\delta_{i j}\right|\right\} \\
&=\left(1-B_{k}^{G E}\right) \sum_{i \in \mathcal{N}}\left\{\left|\mathbb{L}_{k, i}\right|\left(1-B_{i}^{G E}\right) \cdot\right. \\
&\left.\sum_{j \in \mathcal{N}} \hat{\nu}_{i j}\left|\frac{\delta_{i j}}{1+s \delta_{i j}}\right|\left(1-B_{j}^{G E}\right) .\right\}
\end{aligned}
$$

We then have the following result.

Proposition 6. Let $\kappa=2 \chi$ and let $B^{G E}(s)$ be the solution to the fixed point equation

$$
\frac{B_{i}^{G E}}{1-B_{i}^{G E}}=\sum_{j \in \mathcal{N}_{i}} \hat{\nu}_{i j}(s)\left(1-B_{j}^{G E}\right), \quad i \in \mathcal{N},
$$

with

$$
\hat{\nu}_{i j}(s)=\nu_{i j}\left(1+\delta_{i j} s\right), \quad-1 /(1+\kappa \beta) \leq \delta_{i j} \leq 1 .
$$

Then for $0 \leq s \leq \kappa \beta$, we have that

$$
e^{-\chi\left(s+s^{2} / 2\right)} \leq \frac{\left(1-B_{i}^{G E}(s)\right)}{\left(1-B_{i}^{G E}(0)\right)} \leq e^{\chi\left(s+s^{2} / 2\right)}, \quad i \in \mathcal{N} .
$$

Proof: For the proof, we use the same analysis as given to prove Theorem 2 and Corollary 2 in [17]. That is, for $s \in$ $[0, \kappa \beta]$ and $\delta_{i j} \in[-1 /(1+\kappa \beta), 1]$ we have

$$
-1 \leq \frac{\delta_{i j}}{1+s \delta_{i j}} \leq 1
$$

Combining this bound with the fact that $B_{j}(s)$ is the solution to (32), we have that

$$
\sum_{j \in \mathcal{N}} \hat{\nu}_{i j}\left|\frac{\delta_{i j}}{1+s \delta_{i j}}\right|\left(1-B_{j}^{G E}\right) \leq \frac{B_{i}^{G E}}{1-B_{i}^{G E}} .
$$

Combining the above result with Lemma 7, it then follows that

$\left|\frac{d B_{k}^{G E}}{d s}\right| \leq\left(1-B_{k}^{G E}\right) \sum_{i \in \mathcal{N}}\left|\mathbb{L}_{k, i}\right| B_{i}^{G E} \leq\left(1-B_{k}^{G E}\right) \frac{B_{*}^{G E}}{1-B_{*}^{G E}}$.

Recall that $B_{i}^{G E}(s)$ is the solution to

$$
\frac{B_{i}^{G E}(s)}{1-B_{i}^{G E}(s)}=\sum_{j \in \mathcal{N}} \hat{\nu}_{i j}(s)\left(1-B_{j}^{G E}(s)\right), \quad s \in[0, \kappa \beta],
$$

with

$$
\hat{\nu}_{i j}(s)=\nu_{i j}\left(1+\delta_{i j} s\right), \quad-1 /(1+\kappa \beta) \leq \delta_{i j} \leq 1 .
$$

As

$$
\hat{\nu}_{i j}(s) \leq \nu_{i j}(1+s), \quad-1 /(1+\kappa \beta) \leq \delta_{i j} \leq 1
$$

and by Assumption 2 we have that

$$
\sum_{j \in \mathcal{N}_{i}} \nu_{i j} \leq \chi
$$

it follows that

$$
\frac{B_{*}^{G E}}{1-B_{*}^{G E}}<\chi(1+s)
$$

Combining this result with Eq. (33), we obtain that

$$
\left|\frac{d B_{k}^{G E}}{d s}\right| \leq\left(1-B_{k}^{G E}\right) \chi(1+s), \quad s \in[0, \kappa \beta],
$$

and the proposition follows.

We have the following corollary.

Corollary 3. Let $\kappa=2 \chi$. The solution $B(\nu)=$ $\left(B_{1}(\nu), \ldots, B_{N}(\nu)\right)$ to the CSMA fixed point equation given by Eq.(27) then satisfies

$e^{-\chi\left(\kappa \beta+(\kappa \beta)^{2} / 2\right)} \leq \frac{\left(1-B_{i}^{E}(\nu)\right)}{\left(1-B_{i}(\nu)\right)} \leq e^{\chi\left(\kappa \beta+(\kappa \beta)^{2} / 2\right)}, \quad i \in \mathcal{N}$,

where $B^{E}(\nu)=\left(B_{1}^{E}(\nu), \ldots, B_{N}^{E}(\nu)\right)$ is the solution to the Erlang fixed point equation

$$
\frac{B_{i}^{E}}{1-B_{i}^{E}}=\sum_{j \in \mathcal{N}_{i}} \nu_{i j}\left(1-B_{j}^{E}\right), \quad i \in \mathcal{N},
$$

with

$$
\nu_{i j}=\frac{p_{(i, j)}+p_{(j, i)}}{\beta} .
$$

Proof:

Recall that if we vary $\delta_{i j}$ in the interval $[-1 /(1+\kappa \beta), 1]$ and $s$ in the interval $[0, \kappa \beta], \hat{\nu}_{i j}$, then

$$
\hat{\nu}_{i j}(s)=\nu_{i j}\left(1+\delta_{i j} s\right), \quad-1 /(1+\kappa \beta) \leq \delta_{i j} \leq 1 .
$$

will vary in the interval $[1 /(1+\kappa \beta), 1+\kappa \beta]$.

The corollary then follows immediately from Proposition 6 and from Lemma 4 which states that the CSMA fixed point $B(\nu)$ is equal to the a solution $B^{G E}(\hat{\nu})$ to the fixed point equation

$$
B_{i}^{G E}=\sum_{j \in \mathcal{N}_{i}} \hat{\nu}_{i j}\left(1-B_{i}^{G E}\right)\left(1-B_{j}^{G E}\right), \quad i \in \mathcal{N},
$$


where $\hat{\nu}_{i j} \geq 0$ is such that

$$
\frac{1}{1+\kappa \beta} \leq \frac{\hat{\nu}_{i j}}{\nu_{i j}} \leq 1+\kappa \beta, \quad(i, j) \in \mathcal{L},
$$

and $\hat{\nu}_{i j}=0$ if $(i, j) \notin \mathcal{L}$.

The above corollary states that the solution $B(\nu)$ to the CSMA fixed point equation given by Eq.(27) and the solution $B^{E}(\nu)$ to the Erlang fixed point equation become (asymptotically) identical for large $N$, as by Assumption 2 we have hat $\beta$ approaches 0 as $N$ increases. We are going to use this result in Section C-I to prove Proposition 5.

\section{F. Existence of Steady-State Probabilities}

In this section, we show that the family of CSMA policies $(\mathbf{p}, \beta)$ provided in Definition 6 is contained in the set $\mathcal{P}$ of all policies that have well-defined link service rates.

Consider a CSMA policy $\mathbf{p}$ with sensing period $\beta$. Furthermore, recall that $\beta_{l}\left(l^{\prime}\right)$ is the amount of time link $l$ requires to detect that link $l^{\prime}$ has finished transmitting a packet, i.e. $\beta_{l}\left(l^{\prime}\right)$ is the sensing delay of link $l$ for link $l^{\prime}$ (see also Section IV-B).

Recall that we say that a node $i$ is idle if node $i$ is currently neither sending, nor receiving, a data packet. We say that a link $l=(i, j)$ is idle if both node $i$ and $j$ are idle. Otherwise, we say that node $i$ (link $(i, j))$ is busy.

For a given directed link $l=(i, j)$, we refer to node $i$ as the source node of link $l$. We then say that link $l=(i, j)$ is sensed to be idle by its source node, if node $i$ is (a) currently idle and (b) senses node $j$ to be idle. Otherwise, we say that node $i$ senses link $l$ to be busy.

Suppose that at time $t_{0}$ node $i$ has sensed link $l=(i, j)$ to be idle for exactly the duration of a sensing period $\beta$, i.e. node $i$ first detect that link $l$ is idle at time $t_{0}-\beta$. Furthermore, suppose that at time $t_{0}$ node $i$ starts a packet transmission on link $l$. Then we say that link $l$ has been idle in the interval $\left[t_{0}-\beta, t_{0}\right)$.

If at time $t_{0}$, link $l=(i, j)$ just became busy (either because node $i$ started a packet transmission on link $l$, or because a link $l^{\prime} \in \mathcal{I}_{l}$ that interferes with link $l$ started a packet transmission) and that time $t_{1}$ is the first time after time $t_{0}$ that link $l$ is idle again, then we refer to the interval $\left[t_{0}, t_{1}\right)$ as a busy period of link $l$.

Let $y_{l}(t)$ indicate whether link $l$ is busy $\left(y_{l}(t)=1\right)$ or idle $\left(y_{l}(t)=0\right)$. In this section we show that the steady-state probabilities

$$
P\left(y_{i}=0\right)=\lim _{k \rightarrow \infty} P\left(y_{i}(k \beta)=0\right),
$$

for all $i \in \mathcal{L}$, and

$$
P\left(y_{i}=0, y_{j}=0\right)=\lim _{k \rightarrow \infty} P\left(y_{i}(k \beta)=0, y_{j}(k \beta)=0\right),
$$

for all $i \in \mathcal{L}$ and $j \in \mathcal{L}$, exist.

Note that the state of the system at time $t$ can be characterized by the vector $(y(t), z(t))$ where

$$
y(t)=\left(y_{l}(t)\right)_{l \in \mathcal{L}},
$$

indicates for each link $l \in \mathcal{L}$ whether $l$ is busy $\left(y_{l}(t)=1\right)$ or not $\left(y_{l}(t)=0\right)$, and

$$
z(t)=\left(z_{l}(t)\right)_{l \in \mathcal{L}},
$$

indicates the remaining time until node $i$ has the chance to start a packet transmission on link $l$ (if link $l$ is currently idle), or the time until link $l$ becomes idle again (if link $l$ is currently busy).

The existence of the steady-state probabilities $p\left(y_{i}=0\right)$ and $p\left(y_{i}=0, y_{i}=0\right), i, j \in \mathcal{N}$, can easily be established for the special case where (a) all sensing delays are equal to $\beta$, i.e. we have

$$
\beta_{l}\left(l^{\prime}\right)=\beta, \quad l, l^{\prime} \in \mathcal{L},
$$

(b) the sensing times of all nodes are aligned, i.e. all nodes are initial idle and start sensing links at time $t_{0}=0$, and (c) we have that

$$
\beta=\frac{1}{c}
$$

for some integer $c$.

In this case, the system dynamics are given by a finite-state Markov chain $(y(k), z(k)), k \geq 0$, such that

$$
\left(y_{l}(k), z(k)\right)=\left(y_{l}(k \beta), z_{l}(k \beta)\right),
$$

where $y_{l}(k) \in\{0,1\}$ and

$$
z_{l}(k) \in\{\beta, 2 \beta, \ldots, 1,1+\beta\}, \quad l \in \mathcal{L}, k \geq 0 .
$$

Furthermore, the Markov chain has a single-recurrent class containing the state $\left(y^{*}, z^{*}\right)$ given by

$$
y_{l}^{*}=0 \quad \text { and } z_{l}^{*}=\beta, \quad l \in \mathcal{L},
$$

and is aperiodic as the recurrent state $\left(y^{*}, z^{*}\right)$ has a selftransition. It then follows that the above steady-state probabilities exist.

For the general case where not all sensing times are the same, we define a renewal process [12] to establish the existence of the above steady-state probabilities. Without loss of generality we assume for the rest of this section that

(a) for all links $(i, j) \in \mathcal{L}$ we have that $p_{(i, j)}>0$, and

(b) the interference graph consists of one connected component, where the vertex set of the interference graph is equal to $\mathcal{L}$ and there exists an edge between two vertices $l, l^{\prime}$ in the interference graph if link $l$ and $l^{\prime}$ interfere with each other.

1) Recurrent State $\left(y^{*}, z^{*}\right)$ : In the following, we construct a recurrent state $\left(y^{*}, z^{*}\right)$ that we use to define a renewal process for the general case where not all sensing times are the same. To do this, we first iteratively number the links in the following way. At step 1 , let $l_{1}$ be an arbitrary link in $\mathcal{L}$ and let $S_{1}$ be the set of links that have an interference constraint with link $l_{1}$, i.e. we have

$$
S_{1}=\mathcal{I}_{l_{1}} .
$$

In addition set $B_{1}=\left\{l_{1}\right\}$, set $A_{1}=S_{1}$, and set $C_{1}=$ $\mathcal{L} \backslash\left(S_{1} \cup\left\{l_{1}\right\}\right)$, i.e. set $C_{1}$ contains all links except for link $l_{1}$ and the links that interfere with $l_{1}$. We then apply this procedure recursively as follows. Suppose that we are given the sets $A_{k}, B_{k}$, and $C_{k}$, of step $k$. These three sets have the 
following interpretation. Set $B_{k}$ contains all links that have been chosen at step $k$ or an earlier iteration. Set $A_{k}$ contains all links that interfere with at least one link in set $B_{k}$, and set $C_{k}$ contains all links that are not contained in set $A_{k}$ or $B_{k}$. Given these three sets, we proceed at step $k+1$ as follows. If the set $A_{k}$ is empty, then we stop. Otherwise, we pick an arbitrary link from the set $A_{k}$ and label it as $l_{k+1}$. Let $S_{k+1}$ be the set of links in set $C_{k}$ that interfere with link $l_{k+1}$, i.e. we have

$$
S_{k+1}=C_{k} \cap \mathcal{I}_{l_{k+1}} .
$$

Set $B_{k+1}=B_{k} \cup\left\{l_{k+1}\right\}$, set $A_{k+1}=\left(A_{k} \cup S_{k+1}\right) \backslash\left\{l_{k+1}\right\}$, and set $C_{k+1}=C_{k} \backslash S_{k+1}$.

Without loss of generality, we assumed that the interference graph is connected, and the above procedure will terminate after $L$ steps with $A_{L}=C_{L}=\emptyset$.

Having labeled the links as given above, we then construct the following sample path of the system to which we will refer to as sample path $S P^{*}$.

a) Sample Path $S P^{*}: \because$ Suppose that during in the interval $\left[t_{0}, t_{0}+\beta\right)$ all links $l \in \mathcal{L}$ are idle. Then let time $t_{0}^{\prime}$ be given by

$$
t_{0}^{\prime}=t_{0}+\beta,
$$

and let link $l_{1}$ start a packet transmission at time $t_{0}^{\prime}+z_{l_{1}}\left(t_{0}^{\prime}\right)$ while all other links remain idle during in the interval $\left[t_{0}^{\prime}, t_{0}^{\prime}+\right.$ $2 \beta)$ ). Note that in this case the packet transmission of link $l_{1}$ will not experience a collision. Let $t_{1}=t_{0}^{\prime}+z_{l_{1}}\left(t_{0}^{\prime}\right)+1$ be the time when $l_{1}$ finishes its transmission and let all other links remain idle during the interval $\left[t_{0}^{\prime}+2 \beta, t_{1}\right)$.

Then proceed iteratively as follows. Let $t_{k}, k=1, \ldots, N$, be the time when link $l_{k}$ finishes its packet transmission, and let all links be idle in the interval $\left[t_{k}, t_{k}+\beta\right)$. Set

$$
t_{k}^{\prime}=t_{k}+\beta
$$

and let link $l_{k+1}$ start a packet transmission at time $t_{k}^{\prime}+$ $z_{l_{k}}\left(t_{k}^{\prime}\right)$ while all other links remain idle during in the interval $\left[t_{k}^{\prime}, t_{k}^{\prime}+2 \beta\right)$. Let $t_{k+1}=t_{k}^{\prime}+z_{l_{k}}\left(t_{k}^{\prime}\right)+1$ be the time when link $l_{k+1}$ finishes its transmission and let all other links remain idle during the interval $\left[t_{k}^{\prime}+2 \beta, t_{k+1}\right)$.

Let time $t_{L}$ be the time when link $l_{L}$ finishes its packet transmission and let all links to remain idle during the interval $\left[t_{L}, t_{L}+\beta\right)$.

Finally, let

$$
t_{r}=t_{L}+\beta+z_{l_{1}}\left(t_{L}\right)
$$

be the time when link $l_{1}$ has a chance to start a packet transmission in the interval $\left[t_{L}+\beta, t_{L}+2 \beta\right)$, given that the source node of link $l_{1}$ continues to sense link $l_{1}$ to be idle during the interval $\left[t_{r}-\beta, t_{r}\right)$.

Having defined the sample path $S P^{*}$, we show next that the state variable $z\left(t_{r}\right)=\left(z_{l}\left(t_{r}\right)\right)_{l \in \mathcal{L}}$ at the end of the sample path $S P^{*}$ does not depend on the state $z\left(t_{0}^{\prime}\right)$ at time $t_{0}^{\prime}$, but is uniquely determined by the sequence of how all links make their transmission attempts and the fact that all links were idle at time $t_{0}^{\prime}$. To do this, for a scalar $x$ let $\bmod _{\beta}[x]$ be the modulo function given by

$$
\bmod _{\beta}[x]=\left\{\begin{array}{ll}
x-\left\lfloor\frac{x}{\beta}\right\rfloor \beta, & x \geq 0 \\
\left\lceil\frac{|x|}{\beta}|\beta-| x \mid,\right. & x<0 .
\end{array},\right.
$$

and let

$$
\hat{z}_{l}(t)=\bmod _{\beta}\left[z_{l_{1}}(t)-z_{l}(t)\right],
$$

be the difference (offset) between the time when the current active period ends for link $l_{1}$ and $l$. We have the following result.

Lemma 8. Let the time $t_{k}^{\prime}, k=1, \ldots, L$ be as given in the definition of the sample path $S P^{*}$. Then at time $t_{k}^{\prime}$, $k=1, \ldots, L$, for all links $l$ in the set $A_{k} \cup B_{k}$ the offset $\hat{z}_{l}\left(t_{k}^{\prime}\right)$ is given by a function that does not depend on $z\left(t_{0}^{\prime}\right)$, but depends only on the constants $\beta_{l}\left(l^{\prime}\right), l, l^{\prime} \in \mathcal{L}$, and the sequence of the first $k$ links that are activated in the sample path $S P^{*}$.

Proof: As we do not require the transmission time 1 to be divisible by $\beta$, let $\Delta t$ be given by

$$
\Delta t=\bmod _{\beta}(1) \text {. }
$$

We prove the lemma by induction. For the sample path $S P^{*}$, recall that $t_{1}$ is the time when link $l_{1}$ finishes its transmission and $t_{1}^{\prime}$ is given by

$$
t_{1}^{\prime}=t_{1} \beta
$$

It follows that

$$
z_{l_{1}}\left(t_{1}^{\prime}\right)=\beta
$$

and for all links $l$ in the set $A_{1} \cup B_{1}$ we have

$$
z_{l}\left(t_{1}^{\prime}\right)=\beta_{l}\left(l_{1}\right) \text {. }
$$

where $\beta_{l}\left(l_{1}\right)$ is the time link $l$ requires to sense that link $l_{1}$ has finished a packet transmission. It follows that for all links $l$ in the set $A_{1} \cup B_{1}$ we have

$$
\hat{z}_{l}\left(t_{1}^{\prime}\right)=\beta_{l}\left(l_{1}\right),
$$

and the condition given in the lemma is true for $k=1$.

Suppose that the lemma is correct for $k-1 \geq 1$, and let $l_{k}$ be the link $k^{\text {th }}$ link that is activated in the sample path $S P^{*}$. Recall that $t_{k}$ is the time when link $l_{k}$ finishes its transmission and $t_{k}^{\prime}$ is given by

$$
t_{1}^{\prime}=t_{1} \beta .
$$

We first note that when link $l_{1}$ does not interfere with link $l_{k}$, i.e. we have $l_{1} \notin \mathcal{I}_{l_{k}}$, then the transmission of link $l_{k}$ does not affect the offset between $z_{l_{1}}\left(t_{k}^{\prime}\right)$ and $z_{l}\left(t_{k}^{\prime}\right)$ for all links $l \notin \mathcal{I}_{l_{k}}$. Using this observation, we consider the following two cases.

First suppose that $l_{1} \notin \mathcal{I}_{l_{k}}$. Then for all links $l \in A_{k} \cup B_{k}$ such that $l \notin \mathcal{I}_{l_{k}}$, we have that

$$
\hat{z}_{l}\left(t_{k}^{\prime}\right)=\hat{z}_{l}\left(t_{k-1}^{\prime}\right)
$$

and for link $l_{k}$ we have that

$$
\hat{z}_{l_{k}}\left(t_{k}^{\prime}\right)=\bmod _{\beta}\left[\hat{z}_{l_{k}}\left(t_{k-1}^{\prime}\right)+\Delta t\right] \text {. }
$$


For all links $l \in A_{k} \cup B_{k}$ such that $l \in \mathcal{I}_{l_{k}}$, we have that

$$
\hat{z}_{l}\left(t_{k}^{\prime}\right)=\bmod _{\beta}\left[\hat{z}_{l_{k}}\left(t_{k-1}^{\prime}\right)+\Delta t+\beta_{l}\left(l_{k}\right)\right] \text {. }
$$

Next suppose that $l_{1} \in \mathcal{I}_{l_{k}}$. Then for link $l_{k}$ we have that

$$
\hat{z}_{l_{k}}\left(t_{k}^{\prime}\right)=\beta-\beta_{l_{1}}\left(l_{k}\right),
$$

and for all links $l \in A_{k} \cup B_{k}$ such that $l \notin \mathcal{I}_{l_{k}}$, we have that

$$
\hat{z}_{l}\left(t_{k}^{\prime}\right)=\bmod _{\beta}\left[\beta-\beta_{l_{1}}\left(l_{k}\right)+\hat{z}_{l_{k}}\left(t_{k-1}^{\prime}\right)-\hat{z}_{l}\left(t_{k-1}^{\prime}\right)+\Delta t\right] .
$$

For all links $l \in A_{k} \cup B_{k}$ such that $l \in \mathcal{I}_{l_{k}}$, we have that

$$
\hat{z}_{l}\left(t_{k}^{\prime}\right)=\bmod _{\beta}\left[\beta_{l_{1}}\left(l_{k}\right)-\beta_{l}\left(l_{k}\right)\right] .
$$

As by the induction hypothesis $\hat{z}_{l}\left(t_{k}^{\prime}\right)$ does not depend on $z\left(t_{0}^{\prime}\right)$ but only on the constants $\beta_{l}\left(l^{\prime}\right), l, l^{\prime} \in \mathcal{L}$, and the sequence of the first $k$ links that activated in the sample path $S P^{*}$, the statement of the lemma is true for step $k$. The results then follows.

We then have the following lemma.

Lemma 9. Let $t_{0}^{\prime}$ and $t_{r}$ be as given in the definition of the sample path $S P^{*}$. The state $\left(y^{*}, z^{*}\right)=\left(y\left(t_{r}\right), z\left(t_{r}\right)\right)$ in the sample path $S P^{*}$ is given by a function that does not depend on $\left(y\left(t_{0}^{\prime}\right), z\left(t_{0}^{\prime}\right)\right)$, but only on the constants $\beta_{l}\left(l^{\prime}\right), l, l^{\prime} \in \mathcal{L}$, and the sequence of links activated in the sample path $S P^{*}$

Proof: This result follows immediately from Lemma 8 and the fact that

$$
z_{l_{1}}\left(t_{r}\right)=0
$$

and

$$
z_{l}\left(t_{r}\right)=\hat{z}_{l}\left(t_{L}^{\prime}\right), \quad l \neq l_{1}
$$

Next we show that there exists a positive constant $p_{0}$ such that the probability that the above sample path reaches state $\left(y^{*}, z^{*}\right)$ within at most $(1+L)(1+2 \beta)$ time units is lowerbounded by $p_{0}$.

Lemma 10. Let

$$
p_{\max } \triangleq \max _{(i, j) \in \mathcal{L}} p_{(i, j)}, \quad \text { and } \quad p_{\min } \triangleq \min _{(i, j) \in \mathcal{L}} p_{(i, j)} .
$$

Then, the probability that we reach the state $\left(y^{*}, z^{*}\right)$ within $(1+L)(1+2 \beta)$ time units from any given initial state $\left(y\left(t_{0}\right), z\left(t_{0}\right)\right.$ is lower-bounded by

$$
p_{0}=\left(1-p_{\max }\right)^{L(\lceil 1 / \beta\rceil+2)}\left[p_{\min }\left(1-p_{\max }\right)^{L(2+\lceil 1 / \beta\rceil}\right]^{L} .
$$

Proof: Note that from any initial state $\left(y\left(t_{0}\right), z\left(t_{0}\right)\right.$, with probability at least

$$
\left(1-p_{\max }\right)^{L(\lceil 1 / \beta\rceil+2)}
$$

we have for

$$
t_{0}^{\prime}=t_{0}+1+2 \beta
$$

that all links are idle during the interval $\left[t_{0}^{\prime}-\beta, t_{0}^{\prime}\right)$.

Consider the sample path $S P^{*}$. The probability that link $l_{1}$ starts a packet transmission in the interval $\left[t_{0}^{\prime}, t_{0}^{\prime}+\beta\right)$ and all other links remain idle in the interval $\left[t_{0}^{\prime}, t_{0}^{\prime}+2 \beta\right)$, is lowerbounded by

$$
p_{\min }\left(1-p_{\max }\right)^{2 L} \text {. }
$$

The probability that no other link starts a packet transmission in the interval $\left[t_{0}^{\prime}+2 \beta, t_{0}^{\prime}+1+2 \beta\right)$ is lower-bounded by

$$
\left(1-p_{\max }\right)^{L\lceil 1 / \beta\rceil} \text {. }
$$

Let $t_{1}$ be the time when link $l_{1}$ finishes its packet transmission; note that

$$
t_{1}<t_{0}^{\prime}+\beta+1
$$

If all other links remain idle during the interval $\left[t_{0}^{\prime}, t_{0}^{\prime}+1+2 \beta\right)$, then all links are idle during the interval $\left[t_{1}, t_{1}+\beta\right)$.

The result follows by applying the above argument iteratively to the case where link $l_{k}, k=2, \ldots, L$, start a packet transmission under the sample path $S P^{*}$.

2) Renewal Process: Using Lemma 10, we can define a renewal process where renewal epochs are marked by visits to the recurrent state $\left(y^{*}, z^{*}\right)$.

Lemma 11. The expected length of the interval between visits to state $\left(y^{*}, z^{*}\right)$ is bounded, and the visits to the state $\left(y^{*}, z^{*}\right)$ define a renewal process.

We have the following result for the resulting renewal process.

Lemma 12. The renewal process defined by visits to the state $\left(y^{*}, z^{*}\right)$ is either aperiodic, or has a period $\beta / c$ where $c$ is a positive integer.

Proof: The lemma follows immediately from the fact that if $\left(y\left(t_{0}\right), z\left(t_{0}\right)\right)=\left(y^{*}, z^{*}\right)$ then with probability at least $(1-$ $\left.p_{\max }\right)^{L}$ we have that

$$
\left(y\left(t_{0}+\beta\right), z\left(t_{0}+\beta\right)\right)=\left(y^{*}, z^{*}\right) .
$$

Combining the above lemmas, we obtain the following result.

Proposition 7. For every sensing period $\beta>0$, the family of CSMA policies $\mathbf{p}$ is contained in the set $\mathcal{P}$ of all policies that have well-defined link service rates.

Proof: Let $I_{(i, j)}(t)$ be the indicator function for whether link $(i, j)$ is transmitting at time $t$ a packet that does not experience a collision during its entire transmission time. Using Lemma 12, we then we have that (see for example [12])

$$
\lim _{t \rightarrow \infty} \frac{1}{t} \int_{0}^{t} I_{(i, j)}(\tau) d \tau=\lim _{k \rightarrow \infty} P\left(I_{(i, j)}(k \beta)=1\right) .
$$

\section{G. Properties of Balance Equations}

In this section, we characterize the balance equations for the steady-state probabilities

$$
P\left(y_{i}=0\right)=\lim _{k \rightarrow \infty} P\left(y_{i}(k \beta)=0\right), \quad i \in \mathcal{L},
$$

and

$P\left(y_{i}=0, y_{j}=0\right)=\lim _{k \rightarrow \infty} P\left(y_{i}(k \beta)=0, y_{j}(k \beta)=0\right), \quad i, j \in \mathcal{L}$, 
under a CSMA policy $\mathbf{p}$ with sensing period $\beta$.

We are going to use the following notation. If node $i$ is busy at time $t$, i.e. if $y_{i}(t)=1$, let $x_{i}(t), i \in \mathcal{N}$, denote the time until node $i$ becomes idle again, i.e. until $i$ stops sending, or receiving, the current packet transmission. Furthermore, if node $i$ and $j$ are jointly idle at time $t$, i.e. we have that $y_{i}(t)=$ $y_{j}(t)=0$, then let $x_{i j}(t)=x_{j i)}(t)$ be the amount of time that node $i$ and $j$ haven been jointly idle. Note that if node $i$ and $j$ have to be jointly idle for at least the duration of sensing period $\beta$ before node $i$ can potentially start a packet transmission on link $(i, j)$.

1) Preliminary Lemmas: For a given link $l=(i, j)$, recall that $\mathcal{I}_{l}$ be the set of links that interfere with $l$. Suppose that at time $t$ node $i$ and $j$ have been jointly idle for at least $\beta$ time units, i.e. we have that $y_{i}(t)=y_{j}(t)=0$ and $x_{i j}(t) \geq \beta$. Given a CSMA policy $\mathbf{p}$, the probability that node $i$ starts a packet transmission on link $l$ during the interval $(t, t+\beta]$ is then lower-bounded by

$$
p_{(i, j)} \prod_{l^{\prime} \in \mathcal{I}_{l}}\left(1-p_{l^{\prime}}\right)
$$

upper bounded by $p_{(i, j)}$.

Note that from the definition of a CSMA policy, it immediately follows that $p_{(i, j)}$ is an upper-bound on the probability that node $i$ starts a packet transmission on link $l$ during the interval $(t, t+\beta]$. To see that $p_{(i, j)} \prod_{l^{\prime} \in N_{l}}\left(1-p_{l^{\prime}}\right)$ is a lowerbound, we observe the following. Given that at time $t$ node $i$ and $j$ have been jointly idle for at least $\beta$ time units, let $t_{0}$ be the earliest time after $t$ when node $i$ has the chance to start a packet transmission on link $l$, if link $l$ remains idle in the interval $\left(t, t_{0}\right)$. Note that

$$
t_{0} \leq t+\beta
$$

In the worst case, all links $l^{\prime} \in \mathcal{I}_{l}$ have an opportunities to start a packet transmission in the interval $\left[t_{0}-\beta, t_{0}\right)$. In this case, the probability that no link $l^{\prime} \in \mathcal{I}_{l}$ starts a packet transmission during the interval $\left[t_{0}-\beta, t_{0}\right)$, and link $l$ has the opportunity to start a packet transmission at time $t_{0}$ is lower-bounded by

$$
\prod_{l^{\prime} \in \mathcal{I}_{l}}\left(1-p_{l^{\prime}}\right)
$$

and the probability that link $l$ starts a packet transmission in the interval $(t, t+\beta]$ is lower-bounded by $p_{(i, j)} \prod_{l^{\prime} \in \mathcal{I}_{l}}\left(1-p_{l^{\prime}}\right)$.

We have the following result.

Lemma 13. Suppose that at time $t$ node $i$ and $j$ have been jointly idle for at least $\beta$ time units, i.e. we have that $y_{i}(t)=$ $y_{j}(t)=0$ and $x_{i j}(t) \geq \beta$. Then there exists a constant $\kappa_{p}$ such that the probability that the link starts a packet transmission in the interval $(t, t+\beta]$ is lower-bounded by

$$
\frac{1}{1+\kappa_{p} \beta} p_{(i, j)}, \quad \beta \in\left[0,(4 \chi)^{-1}\right]
$$

and upper-bounded by

$$
\left(1+\kappa_{p} \beta\right) p_{(i, j)} \cdot
$$

Proof: For $k \in \mathcal{I}_{l}$ we have that

$$
\left|\frac{d}{d p_{k}} p_{(i, j)} \prod_{l^{\prime} \in \mathcal{I}_{l}}\left(1-p_{l^{\prime}}\right)\right| \leq p_{(i, j)} .
$$

From the mean value theorem, it then follows that

$$
p_{(i, j)}\left(1-\sum_{l^{\prime} \in \mathcal{I}_{l}} p_{l^{\prime}}\right) \leq p_{(i, j)} \prod_{l^{\prime} \in \mathcal{I}_{l}}\left(1-p_{l^{\prime}}\right) .
$$

By Assumption 2 we have that

$$
\sum_{l^{\prime} \in \mathcal{I}_{l}} p_{l^{\prime}} \leq 2 \chi \beta,
$$

and it follows that

$$
p_{(i, j)}(1-2 \chi \beta) \leq p_{(i, j)} \prod_{l^{\prime} \in \mathcal{I}_{l}}\left(1-p_{l^{\prime}}\right) .
$$

Note that for

$$
\kappa_{p} \geq 4 \chi
$$

we have that

$$
\frac{1}{1+\kappa_{p} \beta} \leq(1-2 \chi \beta), \quad \beta \in\left[0,(4 \chi)^{-1}\right] .
$$

The result then follows.

Below, we derive additional lemmas that we are going to use in Section C-G2.

Lemma 14. The probability that a packet transmission experiences a collision is upper-bounded by $4 \chi \beta$.

Proof: Suppose that node $i$ starts a packet transmission on link $l=(i, j)$ at time $t$. Then this packet transmission will experience a collision only if another node starts a packet transmission on a link $l^{\prime} \in \mathcal{I}_{l}$ in the interval $(t-\beta, t+\beta)$. This is because by Assumption 1, we have that for links $l^{\prime} \in \mathcal{I}_{l}$ we have that the sensing delay $\beta_{l}\left(l^{\prime}\right)$ and $\beta_{l^{\prime}}(l)$ is bounded by $\beta$. Furthermore, by Assumption 2 we have that

$$
\sum_{l^{\prime} \in \mathcal{I}_{l}} p_{l^{\prime}} \leq 2 \chi \beta,
$$

and the lemma follows.

Lemma 15. We have

$$
\left.P\left(y_{i}=1, x_{i} \in(0, \beta]\right)=P\left(y_{i}=1, x_{i} \in(1-\beta), 1\right]\right) .
$$

Proof: The above lemma follows immediately from the fact that a packet transmission takes 1 time unit.

Lemma 16. We have

$$
\begin{aligned}
P\left(y_{i}=\right. & \left.1, x_{i} \in(0, \beta]\right) \frac{1}{\beta} \\
& \leq P\left(y_{i}=1\right) \leq P\left(y_{i}=1, x_{i} \in(0, \beta]\right) \frac{1+2 \beta}{\beta} .
\end{aligned}
$$

Proof: The results follows immediately from the fact that the length of a busy period is bounded between 1 (the length of a successful transmission) and $1+2 \beta$ (the maximal length of a collision).

Lemma 17. We have

$$
P\left(y_{i}=1, x_{i} \in(1,1+\beta]\right) \leq P\left(y_{i}=1, x_{i} \in(1-\beta, 1]\right) 4 \chi \beta .
$$

Proof: Note that the event $\left\{y_{i}=1, x_{i} \in(1,1+\beta]\right\}$ indicates that a packet transmission resulted in a collision. By Lemma 14 , the probability of this happening is upper-bounded by $4 \chi \beta$, and the lemma follows. 
Lemma 18. We have

$$
\frac{P\left(y_{i}=0, y_{j}=0, x_{i j} \geq \beta\right)}{P\left(y_{i}=0, y_{j}=0\right)} \geq(1-4 \chi \beta), \quad i, j \in \mathcal{N} .
$$

Proof: Suppose that at time $t$ node $i$ and $j$ have just become jointly idle, and let $T_{t}$ denote the time it takes starting from $t$ until either node $i$ or $j$ become busy. Note that by Assumption 2, we have that

$$
E\left[T_{t}\right] \geq \beta \frac{1}{2 \chi \beta}-\beta=\frac{1}{2 \chi}-\beta .
$$

Furthermore, we have that

$$
\frac{P\left(y_{i}=0, y_{j}=0, x_{i j} \geq \beta\right)}{P\left(y_{i}=0, y_{j}=0\right)}=\frac{E\left[T_{t} \mid T_{t} \geq \beta\right]-\beta}{E\left[T_{t}\right]} .
$$

As

$$
E\left[T_{t} \mid T_{t} \geq \beta\right] \geq E\left[T_{t}\right],
$$

we obtain that

$$
\frac{P\left(y_{i}=0, y_{j}=0, x_{i j} \geq \beta\right)}{P\left(y_{i}=0, y_{j}=0\right)} \geq \frac{E\left[T_{t}\right]-\beta}{E\left[T_{t}\right]} .
$$

Furthermore as

$$
E\left[T_{t}\right] \geq \frac{1}{2 \chi}-\beta
$$

it follows that

$$
\begin{aligned}
\frac{P\left(y_{i}=0, y_{j}=0, x_{i j} \geq \beta\right)}{P\left(y_{i}=0, y_{j}=0\right)} & \geq \frac{E\left[T_{t}\right]-\beta}{E\left[T_{t}\right]} \\
\geq \frac{1 / 2 \chi-2 \beta}{1 / 2 \chi-\beta} & =\frac{1-4 \chi \beta}{1-2 \chi \beta} \geq 1-4 \chi \beta .
\end{aligned}
$$

2) Bounds on the Steady-State Probabilities: In the following, we derive bounds on the steady-state probability $P\left(y_{i}=1\right), i \in \mathcal{N}$. We start with the following lemma.

Lemma 19. For

$$
\beta \in\left[0,(16 \chi)^{-1}\right]
$$

there exists a constant $\kappa_{p}^{\prime}$ such that

$$
\begin{aligned}
& \frac{1}{1+\kappa_{p}^{\prime} \beta} \sum_{j \in \mathcal{N}_{i}} P\left(y_{i}=0, y_{j}=0\right)\left(p_{(i, j)}+p_{(j, i)}\right) \\
& \leq \quad P\left(y_{i}=1, x_{i} \in(1-\beta, 1]\right) \\
& \quad \leq\left(1+\kappa_{p}^{\prime} \beta\right) \sum_{j \in \mathcal{N}_{i}} P\left(y_{i}=0, y_{j}=0\right)\left(p_{(i, j)}+p_{(j, i)}\right) .
\end{aligned}
$$

Proof: Suppose that the system is in steady-state at time $t_{0}$ and that we observe the evolution of the system from time $t_{0}$ to $t_{0}+\beta$. Using lemma 12 , which states that the renewal process is either aperiodic, or has a period of $\beta / c$ where $c$ is a positive integer, it follows that at time $t_{0}+\beta$ the system is again in steady-state. Furthermore, suppose that at time $t_{0}$ nodes $i$ and $j$ have been jointly idle for at least $\beta$ time units, i.e. we have that $y_{i}\left(t_{0}\right)=y_{j}\left(t_{0}\right)=0$ and $x_{i j}\left(t_{0}\right) \geq \beta$. Then by Lemma 13, for $\beta \in\left[0,\left((4 \chi \beta)^{-1}\right)\right.$ there exists a constant $\kappa_{p}$ such that the probability that link $(i, j)$ starts a packet transmission during the interval $\left(t_{0}, t_{0}+\beta\right]$ is bounded between $\frac{1}{\left(1+\kappa_{p} \beta\right)} p_{(i, j)}$ and $\left(1+\kappa_{p} \beta\right) p_{(i, j)}$. Furthermore, these two bounds provided by Lemma 13 are independent of the states of all other links, and hence independent of states of the states of nodes other than node $i$ and $j$. By Lemma 14 the probability that this transmission will result in a collision is upper-bounded by $4 \chi \beta$. When the transmission does not result in a collision, then at $t_{0}+\beta$ the remaining time until node $i$ finishes the packet transmission will be in the interval $(1-\beta, 1]$, i.e. we have $x_{i}\left(t_{0}+\beta\right) \in(1-\beta, 1]$.

Combining the above results, we obtain that

$$
\begin{aligned}
\frac{1-4 \chi \beta}{1+\kappa_{p} \beta} \sum_{j \in \mathcal{N}_{i}} P\left(y_{i}=0, y_{j}\right. & \left.=0, x_{i j} \geq \beta\right)\left(p_{(i, j)}+p_{(j, i)}\right) \\
\leq P\left(y_{i}\right. & \left.=1, x_{i} \in(1-\beta, 1]\right)
\end{aligned}
$$

and

$$
\begin{aligned}
P\left(y_{i}=1,\right. & \left.x_{i} \in(1-\beta, 1]\right) \\
\leq & \left(1+\kappa_{p} \beta\right) \sum_{j \in \mathcal{N}_{i}} P\left(y_{i}=0, y_{j}=0\right)\left(p_{(i, j)}+p_{(j, i)}\right) \\
& +P\left(y_{i}=1, x_{i} \in(1,1+\beta]\right),
\end{aligned}
$$

where the last term $P\left(y_{i}=1, x_{i} \in(1,1+\beta]\right)$ accounts for the probability that at time $t_{0}$ node $i$ is experiencing a collision that will last another $t_{c}$ time units with $t_{c} \in(1,1+\beta]$.

Using Lemma 18, we obtain for the first inequality that

$$
\begin{array}{r}
\frac{(1-4 \chi \beta)^{2}}{1+\kappa_{p} \beta} \sum_{j \in \mathcal{N}_{i}} P\left(y_{i}=0, y_{j}=0\right)\left(p_{(i, j)}+p_{(j, i)}\right) \\
\leq P\left(y_{i}=1, x_{i} \in(1-\beta, 1]\right) .
\end{array}
$$

Furthermore, using Lemma 17 we obtain that

$$
\begin{gathered}
P\left(y_{i}=1, x_{i} \in(1-\beta, 1]\right) \\
\leq \quad\left(1+\kappa_{p} \beta\right) \sum_{j \in \mathcal{N}_{i}} P\left(y_{i}=0, y_{j}=0\right)\left(p_{(i, j)}+p_{(j, i)}\right) \\
\quad+P\left(y_{i}=1, x_{i} \in(1-\beta, 1]\right) 4 \chi \beta,
\end{gathered}
$$

or

$$
\begin{aligned}
P\left(y_{i}\right. & \left.=1, x_{i} \in(1-\beta, 1]\right) \\
& \leq \frac{1+\kappa_{p} \beta}{1-4 \chi \beta} \sum_{j \in \mathcal{N}_{i}} P\left(y_{i}=0, y_{j}=0\right)\left(p_{(i, j)}+p_{(j, i)}\right) .
\end{aligned}
$$

Note that for $\beta \in\left[0,(16 \chi)^{-1}\right]$ and $\kappa_{p}^{\prime} \geq 2\left(\kappa_{p}+8 \chi\right)$ we have that

$$
\frac{1}{1+\kappa_{p}^{\prime} \beta} \leq \frac{1-8 \chi \beta}{1+\kappa_{p} \beta} \leq \frac{(1-4 \chi \beta)^{2}}{1+\kappa_{p} \beta} .
$$

The lemma then follows.

Using Lemma 19, we obtain the following bound for the steady-state probability $P\left(y_{i}=1\right), i \in \mathcal{N}$.

Lemma 20. For $\beta \in\left[0,(16 \chi)^{-1}\right]$, there exists a constant $\kappa_{s}$ such that

$$
\begin{aligned}
& \frac{1}{1+\kappa_{s} \beta} \sum_{j \in \mathcal{N}_{i}} P\left(y_{i}=0, y_{j}=0\right) \nu_{i j} \\
& \leq \quad P\left(y_{i}=1\right) \\
& \quad \leq\left(1+\kappa_{s} \beta\right) \sum_{j \in \mathcal{N}_{i}} P\left(y_{i}=0, y_{j}=0\right) \nu_{i j},
\end{aligned}
$$

where

$$
\nu_{i j}=\frac{p_{(i, j)}+p_{(j, i)}}{\beta} .
$$



have

Proof: Using Lemma 15-19, for $\beta \in\left[0,(16 \chi)^{-1}\right]$ we

$$
\begin{aligned}
P\left(y_{i}=1, x_{i} \in(1-\beta, 1]\right) \frac{1}{\beta} & \\
\leq & P\left(y_{i}=1\right) \\
& \leq P\left(y_{i}=1, x_{i} \in(1-\beta, 1]\right) \frac{1+2 \beta}{\beta},
\end{aligned}
$$

and there exists a constant $\kappa_{p}^{\prime}$ such that

$$
\begin{aligned}
& \frac{1}{1+\kappa_{p}^{\prime} \beta} \sum_{j \in \mathcal{N}_{i}} P\left(y_{i}=0, y_{j}=0\right)\left(p_{(i, j)}+p_{(j, i)}\right) \\
& \leq P\left(y_{i}=1, x_{i} \in(1-\beta, 1]\right) \\
& \quad \leq\left(1+\kappa_{p}^{\prime} \beta\right) \sum_{j \in \mathcal{N}_{i}} P\left(y_{i}=0, y_{j}=0\right)\left(p_{(i, j)}+p_{(j, i)}\right)
\end{aligned}
$$

Combing the above results, we have that

$$
\begin{aligned}
& \frac{1}{1+\kappa_{p}^{\prime} \beta} \sum_{j \in \mathcal{N}_{i}} P\left(y_{i}=0, y_{j}=0\right) \nu_{i j} \\
& \quad \leq P\left(y_{i}=1\right) \\
& \quad \leq\left(1+\kappa_{p}^{\prime} \beta\right)(1+2 \beta) \sum_{j \in \mathcal{N}_{i}} P\left(y_{i}=0, y_{j}=0\right) \nu_{i j},
\end{aligned}
$$

where

$$
\nu_{i j}=\frac{p_{(i, j)}+p_{(j, i)}}{\beta} .
$$

Note that for $\beta \in\left[0,(16 \chi)^{-1}\right]$ and

we have that

$$
\kappa_{s} \geq \kappa_{p}^{\prime}+2+\frac{\kappa_{p}^{\prime}}{8 \chi}
$$

$$
\left(1+\kappa_{p}^{\prime} \beta\right)(1+2 \beta) \leq 1+\kappa_{s} \beta .
$$

The lemma then follows.

\section{H. Characterization of the steady-state probabilities}

In this section, we characterize the steady-state probabilities

$$
\bar{B}_{i}=1-P\left(y_{i}=0\right), \quad i \in \mathcal{N},
$$

that a node $i$ is busy under a CSMA policy $\mathbf{p}$ with sensing period $\beta$, using the same analysis as given by Hajek and Krishna in Section 3 and 4 of the reference [17] with only minor changes.

Throughout this section, we set

$$
\nu_{i j}=\frac{p_{(i, j)}+p_{(j, i)}}{\beta}, \quad i, j \in \mathcal{N},
$$

with $\nu_{i j}=0$ if $(i, j) \notin \mathcal{L}$ and $(j, i) \notin \mathcal{L}$.

Note that by Lemma 20 there exists a constant $\kappa_{s}$ such that

$$
\begin{aligned}
& \frac{1}{1+\kappa_{s} \beta} \sum_{j \in \mathcal{N}_{i}} P\left(y_{i}=0, y_{j}=0\right) \nu_{i j} \\
& \quad \leq P\left(y_{i}=1\right) \\
& \quad \leq\left(1+\kappa_{s} \beta\right) \sum_{j \in \mathcal{N}_{i}} P\left(y_{i}=0, y_{j}=0\right) \nu_{i j} .
\end{aligned}
$$

We have the following result.
Lemma 21. Let $\kappa_{s}$ be the constant of Lemma 20. Then for $k, l \in \mathcal{N}$ we have that

$$
\begin{aligned}
& \frac{1}{1+\kappa_{s} \beta} \sum_{j \neq k, l} P\left(y_{k}=0, y_{j}=0, y_{l}=0\right) \nu_{k j} \leq \\
& \leq P\left(y_{k}=1, y_{l}=0\right) \\
& \quad \leq\left(1+\kappa_{s} \beta\right) \sum_{j \neq k, l} P\left(y_{k}=0, y_{j}=0, y_{l}=0\right) \nu_{k j} .
\end{aligned}
$$

Proof: Note that we have

$$
P\left(y_{k}=1, y_{l}=0\right)=P\left(y_{k}=1 \mid y_{l}=0\right) P\left(y_{l}=0\right),
$$

and

$$
\begin{aligned}
& P\left(y_{k}=0, y_{j}=0, y_{l}=0\right) \\
& \quad=P\left(y_{k}=0, y_{j}=0 \mid y_{l}=0\right) P\left(y_{l}=0\right) .
\end{aligned}
$$

Therefore, to obtain the result, it suffices to show that

$$
\begin{aligned}
& \frac{1}{1+\kappa_{s} \beta} \sum_{j \neq k, l} P\left(y_{k}=0, y_{j}=0 \mid y_{l}=0\right) \nu_{k j} \\
& \leq P\left(y_{k}=1 \mid y_{l}=0\right) \\
& \quad \leq\left(1+\kappa_{s} \beta\right) \sum_{j \neq k, l} P\left(y_{k}=0, y_{j}=0 \mid y_{l}=0\right) \nu_{k j} .
\end{aligned}
$$

The above inequalities are obtained by the same argument as given in the proof for Lemma 20.

We then have the following result.

Proposition 8. Let $\kappa_{s}$ be the constant of Lemma 20. For $k, l \in$ $\mathcal{N}$ we then have that

$$
\begin{aligned}
\frac{1}{1+2 r_{p}}\left(\frac{1}{1+\kappa_{s} \beta}\right)^{2 N} & \leq \frac{P\left(y_{k}=0, y_{l}=0\right)}{P\left(y_{k}=0\right) P\left(y_{l}=0\right)} \\
& \leq\left(1+\kappa_{s} \beta\right)^{2 N}\left(1+2 r_{p}\right),
\end{aligned}
$$

with $r_{p} \triangleq \frac{p_{\max }}{\beta}$ and $p_{\max }$ is as given in Assumption 2 .

Proof: Let $Z_{i}$ be the steady-stated probability $P\left(y_{i}=0\right)$ that node $i$ is idle, let $Z_{i j}$ be the steady-stated probability $P\left(y_{i}=0, y_{j}=0\right)$ that nodes $i$ and $j$ are jointly idle, and let $Z_{i j k}$ be the steady-stated probability $P\left(y_{i}=0, y_{j}=0, y_{k}=\right.$ 0 ) that nodes $i, j$, and $k$, are jointly idle.

We use a proof by induction on the number of nodes in the network, as given in [17]. For a network with $N=1$ node the proposition is trivially true, and suppose that $N \geq 2$.

Using Lemma 21, we have that

$$
\begin{aligned}
& \frac{1}{1+\kappa_{s} \beta}\left(Z_{k l}+\sum_{j \neq k, l} Z_{j k l} \nu_{j k}\right) \\
& \quad \leq Z_{l} \leq\left(1+\kappa_{s} \beta\right)\left(Z_{k l}+\sum_{j \neq k, l} Z_{j k l} \nu_{j k}\right) .
\end{aligned}
$$

Furthermore, starting with the equation

$$
1=P\left(y_{k}=0\right)+P\left(y_{k}=1\right)
$$

and using the result from Lemma 20, which states that

$$
\begin{aligned}
& \frac{1}{1+\kappa_{s} \beta}\left(\sum_{j \in \mathcal{N}_{k}} P\left(y_{k}=0, y_{j}=0\right) \nu_{k j}\right) \\
& \leq P\left(y_{k}=1\right) \\
& \quad \leq\left(1+\kappa_{s} \beta\right)\left(\sum_{j \in \mathcal{N}_{k}} P\left(y_{k}=0, y_{j}=0\right) \nu_{k j}\right),
\end{aligned}
$$


we obtain

$$
\begin{aligned}
& \frac{1}{1+\kappa_{s} \beta}\left(P\left(y_{k}=0\right)+\sum_{j \in \mathcal{N}_{k}} P\left(y_{k}=0, y_{j}=0\right) \nu_{k j}\right) \\
& \leq 1 \\
& \leq\left(1+\kappa_{s} \beta\right)\left(P\left(y_{k}=0\right)+\sum_{j \in \mathcal{N}_{k}} P\left(y_{k}=0, y_{j}=0\right) \nu_{k j}\right) \text {. }
\end{aligned}
$$

Combining the above inequalities, we obtain by the same approach as in [17] that

$$
\begin{aligned}
& \frac{1}{\left(1+\kappa_{s} \beta\right)^{2}} \frac{\left(Z_{k}+Z_{k l} \nu_{k l}\right) Z_{k l}+\sum_{j \neq k, l} Z_{j k} Z_{k l} \nu_{j k}}{Z_{k} Z_{k l}+\sum_{j \neq k, l} Z_{k} Z_{j k l} \nu_{j k}} \\
& \leq \frac{P\left(y_{k}=0, y_{l}=0\right)}{P\left(y_{k}=0\right) P\left(y_{l}=0\right)} \leq \\
& \quad\left(1+\kappa_{s} \beta\right)^{2} \frac{\left(Z_{k}+Z_{k l} \nu_{k l}\right) Z_{k l}+\sum_{j \neq k, l} Z_{j k} Z_{k l} \nu_{j k}}{Z_{k} Z_{k l}+\sum_{j \neq k, l} Z_{k} Z_{j k l} \nu_{j k}} .
\end{aligned}
$$

Using the fact that $Z_{k l} \leq Z_{k}$ and by Assumption 2 we have

$$
0 \leq \nu_{i j} \leq 2 r_{p}
$$

it follows that

$$
1 \leq \frac{Z_{k}+Z_{k l} \nu_{k l}}{Z_{k}} \leq 1+2 r_{p}
$$

and

$$
Z_{k} \leq Z_{k}+Z_{k l} \nu_{k l} \leq\left(1+2 r_{p}\right) Z_{k}
$$

Furthermore, from the induction hypotheses applied to the network with $N-1$ nodes, we obtain, by deleting node $k$,

$$
\begin{aligned}
\frac{1}{1+2 r_{p}}\left(\frac{1}{1+\kappa_{s} \beta}\right)^{2(N-1)} & \leq \frac{Z_{j k} Z_{k l}}{Z_{k} Z_{j k l}} \\
& \leq\left(1+\kappa_{s} \beta\right)^{2(N-1)}\left(1+2 r_{p}\right)
\end{aligned}
$$

and

$$
\begin{aligned}
\frac{1}{1+2 r_{p}} & \left(\frac{1}{1+\kappa_{s} \beta}\right)^{2(N-1)} Z_{k} Z_{j k l} \leq Z_{j k} Z_{k l} \\
& \leq\left(1+\kappa_{s} \beta\right)^{2(N-1)}\left(1+2 r_{p}\right) Z_{k} Z_{j k l} .
\end{aligned}
$$

Using Eq. (35) and (36) in Eq. (34), we obtain

$$
\begin{gathered}
\frac{\left(Z_{k} Z_{k l}+\frac{1}{1+2 r_{p}}\left(\frac{1}{1+\kappa_{s} \beta}\right)^{2(N-1)} \sum_{j \neq k, l} Z_{k} Z_{j k l} \nu_{j k}\right)}{\left(1+\kappa_{s} \beta\right)^{2}\left(Z_{k} Z_{k l}+\sum_{j \neq k, l} Z_{k} Z_{j k l} \nu_{j k}\right)} \\
\leq \frac{P\left(y_{k}=0, y_{l}=0\right)}{P\left(y_{k}=0\right) P\left(y_{l}=0\right)} \leq \sum_{j \neq k, l} Z_{k} Z_{j} \\
\text { and } \quad \\
\frac{\left.1+\kappa_{s} \beta\right)^{2}\left(1+2 r_{p}\right) \frac{\left(Z_{k} Z_{k l}+\left(1+\kappa_{s} \beta\right)^{2(N-1)}\right.}{1+2 r_{p}}\left(\frac{1}{1+\kappa_{s} \beta}\right)^{2 N} \leq \frac{P\left(y_{k}=0, y_{l}=0\right)}{P\left(y_{k}=0\right) P\left(y_{l}=0\right)}}{\leq} \leq\left(1+\kappa_{s} \beta\right)^{2 N}\left(1+2 r_{p}\right) .
\end{gathered}
$$

The result then follows.

We then obtain the following corollary.
Corollary 4. Let $\kappa_{s}$ be the constant of Lemma 20, and let $\bar{B}_{i}$ be the actual steady-state probability that node $i$ is busy. Then

$$
\frac{\bar{B}_{i}}{1-\bar{B}_{i}}=\sum_{j \in \mathcal{N}_{i}} \tilde{\nu}_{i j}\left(1-\bar{B}_{j}\right)
$$

where $\tilde{\nu}_{i j}$ is such that

$\frac{1}{1+2 r_{p}}\left(\frac{1}{1+\kappa_{s} \beta}\right)^{2 N+1} \leq \frac{\tilde{\nu}_{i j}}{\nu_{i j}} \leq\left(1+\kappa_{s} \beta\right)^{2 N+1}\left(1+2 r_{p}\right)$, where $r_{p}$ is as given in Proposition 8.

The above results follows immediately from Proposition 8 and Lemma 20. Using the above Corollary 4, we obtain the following result.

Corollary 5. Let $\kappa_{s}$ be the constant of Lemma 20. Then there exists an integer $N_{0}$ such that for $N>N_{0}$ the actual steadystate probability

$$
\sigma_{i}=1-\bar{B}_{i}, \quad i \in \mathcal{N},
$$

that node $i$ is idle in a network of size $N$ satisfies

$$
\left(1-B_{i}^{E}(\nu)\right) e^{-\chi\left(r+r^{2} / 2\right)} \leq \sigma_{i} \leq\left(1-B_{i}^{E}(\nu)\right) e^{\chi\left(r+r^{2} / 2\right)},
$$

where $B^{E}(\nu)=\left(B_{1}^{E}(\nu), \ldots, B_{N}^{E}(\nu)\right)$ is the solution to the Erlang fixed point equation given by

$$
\frac{B_{i}^{E}}{1-B_{i}^{E}}=\sum_{j \in \mathcal{N}_{i}} \nu_{i j}\left(1-B_{j}^{E}\right), \quad i \in \mathcal{N},
$$

where

$$
r=2\left[(2 N+1)\left(\kappa_{s} \beta\right)+2 r_{p}\right]
$$

and $r_{p}$ is given in Proposition 8.

Proof: Note that

$$
\left(1+\kappa_{s} \beta\right)^{2 N+1}\left(1+2 r_{p}\right) \leq e^{(2 N+1)\left(\kappa_{s} \beta\right)+2 r_{p}} .
$$

and

$$
\frac{1}{1+2 r_{p}}\left(\frac{1}{1+\kappa_{s} \beta}\right)^{2 N+1} \geq e^{-\left[(2 N+1)\left(\kappa_{s} \beta\right)+2 r_{p}\right]} .
$$

Furthermore, recall that $r_{p}=\frac{p_{\max }}{\beta}$, and, by Assumption 2,

$$
\lim _{N \rightarrow \infty} \frac{p_{\max }^{(N)}}{\beta^{(N)}}=0, \quad \text { and } \quad \lim _{N \rightarrow \infty} \beta^{(N)} N=0 .
$$

It follows that there exists an integer $N_{0}$ such that for $N>N_{0}$ we have

$$
e^{(2 N+1)\left(\kappa_{s} \beta\right)+2 r_{p}}<2
$$

$$
e^{(2 N+1)\left(\kappa_{s} \beta\right)+2 r_{p}}<1+2\left[(2 N+1)\left(\kappa_{s} \beta\right)+2 r_{p}\right],
$$

where we used the fact that the function $e^{x}$ is convex and that $\lim _{x \rightarrow 0} e^{x}=1$. Similarly, for $N>N_{0}$ we have

$$
\frac{1}{1+2\left[(2 N+1)\left(\kappa_{s} \beta\right)+2 r_{p}\right]}<e^{-\left[(2 N+1)\left(\kappa_{s} \beta\right)+2 r_{p}\right]} .
$$

Using Corollary 4 , for $N>N_{0}$ we then have

$$
\frac{\bar{B}_{i}}{1-\bar{B}_{i}}=\sum_{j \in \mathcal{N}_{i}} \tilde{\nu}_{i j}\left(1-\bar{B}_{j}\right),
$$


where $\tilde{\nu}_{i j}$ is such that

$$
\begin{aligned}
& \frac{1}{1+2\left[(2 N+1)\left(\kappa_{s} \beta\right)+2 r_{p}\right]} \\
& \leq \frac{\tilde{\nu}_{i j}}{\nu_{i j}} \leq 1+2\left[(2 N+1)\left(\kappa_{s} \beta\right)+2 r_{p}\right] .
\end{aligned}
$$

Using the same argument as given in the proof of Proposition 6 and Corollary 3 in Appendix $\mathrm{C}$-E, we then obtain the result of this corollary.

\section{Proof of Proposition 5}

In this section, we combine the results of $\mathrm{Sec}$ tions $\mathrm{C}-\mathrm{E}$ and $\mathrm{C}-\mathrm{H}$ to prove Proposition 5.

Proof: Consider a CSMA policy $\mathbf{p}$ for a wireless network consisting of $N$ nodes and set

$$
\nu_{i j}=\frac{p_{(i, j)}+p_{(j, i)}}{\beta}, \quad i, j \in \mathcal{N} .
$$

Let $B_{i}(\nu), i=1, \ldots, N$, be the CSMA fixed point given by Eq. (27), and let $\sigma_{i}(\mathbf{p})$ be the actual steady-state probability that node $i$ is idle under the CSMA policy $\mathbf{p}$. Then by Corollary 5, there exists a integer $N_{0}$ such that for $N>N_{0}$ we have that the steady-state probabilities $\sigma_{i}, i \in \mathcal{N}$, satisfy

$\left(1-B_{i}^{E}(\nu)\right) e^{-\chi\left(r+r^{2} / 2\right)} \leq \sigma_{i}(\mathbf{p}) \leq\left(1-B_{i}^{E}(\nu)\right) e^{\chi\left(r+r^{2} / 2\right)}$,

where $B_{i}^{E}(\nu)$ is the solution to the Erlang fixed point given by the equations

$$
\frac{B_{i}^{E}}{1-B_{i}^{E}}=\sum_{j \in \mathcal{N}_{i}} \nu_{i j}\left(1-B_{j}^{E}\right), \quad i \in \mathcal{N},
$$

and $r=2\left[(2 N+1)\left(\kappa_{s} \beta\right)+2 r_{p}\right]$ is as given in Corollary 5 .

Let $B(\nu)$ be the CSMA fixed point given by Eq. (27) and recall the relation that

$$
\rho_{i}(\mathbf{p})=1-B_{i}(\nu) .
$$

Then by Corollary 3 we have that there exists a constant $\kappa$ such that

$$
\begin{aligned}
& \left(1-B_{i}^{E}(\nu)\right) e^{-\chi\left(\kappa \beta+(\kappa \beta)^{2} / 2\right)} \\
& \quad \leq \rho_{i}(\mathbf{p}) \leq\left(1-B_{i}^{E}(\nu)\right) e^{\chi\left(\kappa \beta+(\kappa \beta)^{2} / 2\right)} .
\end{aligned}
$$

Combining the above results, we immediately obtain Proposition 5 .

\section{APPENDIX D}

\section{PROOF OF THEOREM 4}

Proof: Recall that the set $\mathcal{C}_{\infty}$ is given by

$\mathcal{C}_{\infty}=\left\{\left\{\lambda^{(N)}\right\}_{N \geq 1} \in \mathcal{A} \mid \lim \sup _{N \rightarrow \infty}\left(\max _{i=1, \ldots, N} \Lambda_{i}^{(N)}\right)<1\right\}$,

and that in Theorem 4 we consider a sequence of networks $\left\{\mathcal{N}^{(N)}, \mathcal{L}^{(N)}\right\}_{N \geq 1}$ and a sequence of sensing periods $\left(\beta^{(N)}\right)_{N \geq 1}$ such that

$$
\lim _{N \rightarrow \infty} N \beta^{(N)}=0 .
$$

Theorem 4 then states that for every sequence $\lambda^{(N)} \in \mathcal{C}$ there exists a sequence of CSMA policies $\left\{\mathbf{p}^{(N)}\right\}_{N \geq 1}$ that asymptotically stabilizes the network, i.e. we have

$$
\liminf _{N \rightarrow \infty}\left(\min _{(i, j) \in \mathcal{L}^{(N)}} \frac{\mu_{(i, j)}\left(\mathbf{p}^{(N)}\right)}{\lambda_{(i, j)}^{(N)}}\right)>1 .
$$

We prove Theorem 4 as follows. By definition, for each sequence $\left\{\lambda^{(N)}\right\}_{N \geq 1} \in \mathcal{C}_{\infty}$ there exists a scalar $\bar{\Lambda}<1$ and an integer $\bar{N}$ such that for $N \geq \bar{N}$ we have

$$
\Lambda_{i}^{(N)} \leq \bar{\Lambda}, \quad i=1, \ldots, N .
$$

Let then $\Lambda^{*}$ be given by

$$
\Lambda^{*} \triangleq 1-\frac{1-\bar{\Lambda}}{2}<1
$$

and let

$$
\gamma \triangleq \frac{\Lambda^{*}}{\bar{\Lambda}}>1
$$

Using these definitions, let

$$
\begin{aligned}
& \bar{\lambda}_{(i, j)}^{(N)} \triangleq \gamma \lambda_{(i, j)}^{(N)}, \quad(i, j) \in \mathcal{L}, \quad \text { and } \\
& \bar{\Lambda}_{i}^{(N)} \triangleq \sum_{j \in \mathcal{N}_{i}^{(N)}}\left[\bar{\lambda}_{(i, j)}^{(N)}+\bar{\lambda}_{(j, i)}^{(N)}\right], \quad i \in \mathcal{N}^{(N)} .
\end{aligned}
$$

For all $i \in \mathcal{N}^{(N)}$, we then have

$$
\bar{\Lambda}_{i}^{(N)} \leq \Lambda^{*}, \quad N \geq \bar{N} .
$$

As $\lim _{N \rightarrow \infty} \beta^{(N)}=0$ and $\lim _{\beta \downarrow 0} \tau\left(G^{+}(\beta)\right)=1$ (see (6)), there exists an integer $N_{0}$ such that for $N \geq N_{0}$ we have

$$
\bar{\Lambda}_{i}^{(N)} \leq \Lambda^{*}<\tau\left(G^{+}\left(\beta^{(N)}\right)\right) e^{-\left(G^{+}\left(\beta^{(N)}\right)\right)}, \quad i=1, \ldots, N .
$$

Using this result, for a given network size $N \geq N_{0}$ let $G_{i}^{(N)} \in\left[0, G^{+}\left(\beta^{(N)}\right)\right)$ be such that

$$
e^{\left(G_{i}^{(N)}-G^{+}(\beta)\right)} \tau\left(G_{i}^{(N)}\right) e^{-G^{+}(\beta)}=\bar{\Lambda}_{i}^{(N)}
$$

and let

$$
\rho_{i}^{(N)}=\frac{\beta^{(N)}}{\beta^{(N)}+1-e^{-G_{i}^{(N)}}} .
$$

Such a $G_{i}^{(N)}$ exists as shown in the proof of Theorem 2 .

For $N \geq N_{0}$, consider then the CSMA policy $\mathbf{p}^{(N)}$ given by

$$
p_{(i, j)}^{(N)} \triangleq \frac{\bar{\lambda}_{(i, j)}^{(N)}}{\rho_{i}^{(N)} \rho_{j}^{(N)}} \beta^{(N)} e^{2 G^{+}\left(\beta^{(N)}\right)}, \quad(i, j) \in \mathcal{L} .
$$

Using the proof of Theorem 2 , we then have for $N \geq N_{0}$ that

$$
\bar{\lambda}_{(i, j)}^{(N)}<\tau_{(i, j)}\left(\mathbf{p}^{(N)}\right), \quad(i, j) \in \mathcal{L}^{(N)} .
$$

Also, using Theorem 3, the approximation $\tau_{(i, j)}\left(\mathbf{p}^{(N)}\right)$ of the service rate of link $(i, j)$ is asymptotically accurate as $N$ increases if the sequence $\left\{\mathbf{p}^{(N)}\right\}_{N \geq N_{0}}$ satisfies Assumption 2. 
Next, we complete the proof of Theorem 4 assuming Assumption 2 holds and then confirm that it does.

$$
\begin{aligned}
& \liminf _{N \rightarrow \infty}\left(\min _{(i, j) \in \mathcal{L}^{(N)}} \frac{\mu_{(i, j)}\left(\mathbf{p}^{(N)}\right)}{\lambda_{(i, j)}^{(N)}}\right) \\
& =\liminf _{N \rightarrow \infty}\left(\min _{(i, j) \in \mathcal{L}^{(N)}} \frac{\tau_{(i, j)}\left(\mathbf{p}^{(N)}\right)}{\lambda_{(i, j)}^{(N)}} \frac{\mu_{(i, j)}\left(\mathbf{p}^{(N)}\right)}{\tau_{(i, j)}\left(\mathbf{p}^{(N)}\right)}\right) \\
& =\liminf _{N \rightarrow \infty}\left(\min _{(i, j) \in \mathcal{L}^{(N)}} \frac{\tau_{(i, j)}\left(\mathbf{p}^{(N)}\right)}{\lambda_{(i, j)}^{(N)}}\right) \\
& \geq \liminf _{N \rightarrow \infty}\left(\gamma \min _{(i, j) \in \mathcal{L}^{(N)}} \frac{\tau_{(i, j)}\left(\mathbf{p}^{(N)}\right)}{\bar{\lambda}_{(i, j)}^{(N)}}\right) \\
& \geq \gamma>1 .
\end{aligned}
$$

To verify Assumption 2 for the sequence $\left\{\mathbf{p}^{(N)}\right\}_{N \geq N_{0}}$, we first show that for

$$
p_{\max }^{(N)}=\max _{(i, j) \in \mathcal{L}^{(N)}} p_{(i, j)}^{(N)}
$$

we have that

$$
\lim _{N \rightarrow \infty} \frac{p_{\max }^{(N)}}{\beta^{(N)}}=0
$$

Note that by definition, we have for $(i, j) \in \mathcal{L}$ that

$$
\begin{aligned}
& \frac{p_{(i, j)}^{(N)}}{\beta^{(N)}} e^{-2 G^{+}\left(\beta^{(N)}\right)} \\
& =\bar{\lambda}_{(i, j)}^{(N)} \frac{\left(\beta^{(N)}+1-e^{\left.-G_{i}^{(N)}\right)\left(\beta^{(N)}+1-e^{-G_{j}^{(N)}}\right)}\right.}{\left(\beta^{(N)}\right)^{2}} \\
& \leq \max _{(k, l) \in \mathcal{L}}\left(\bar{\lambda}_{(k, l)}^{(N)}\right) \frac{\left(\beta^{(N)}+1-e^{\left.-G_{i}^{(N)}\right)\left(\beta^{(N)}+1-e^{-G_{j}^{(N)}}\right)}\right.}{\left(\beta^{(N)}\right)^{2}} \\
& \leq \gamma \max _{(k, l) \in \mathcal{L}}\left(\lambda_{(k, l)}^{(N)}\right) \frac{\left(\beta^{(N)}+1-e^{-G_{i}^{(N)}}\right)\left(\beta^{(N)}+1-e^{-G_{j}^{(N)}}\right)}{\left(\beta^{(N)}\right)^{2}}
\end{aligned}
$$

where $\gamma$ is the constant of Eq. (37).

Suppose that we can show that there exists a constant $\kappa$ and an integer $N_{0}$ such that for all $N \geq N_{0}$ we have that

$$
G_{i}^{(N)} \leq \kappa \beta^{(N)}, \quad i \in \mathcal{N} .
$$

In this case, for all $(i, j) \in \mathcal{L}$ we have

$\frac{p_{(i, j)}^{(N)}}{\beta^{(N)}} \leq \gamma \max _{(k, l) \in \mathcal{L}}\left(\lambda_{(k, l)}^{(N)}\right)\left(\frac{\beta^{(N)}+1-e^{-\kappa \beta^{(N)}}}{\left(\beta^{(N)}\right)}\right)^{2} e^{2 G^{+}\left(\beta^{(N)}\right)}$, and it follows that

$\frac{p_{\max }^{(N)}}{\beta^{(N)}} \leq \gamma \max _{(k, l) \in \mathcal{L}}\left(\lambda_{(k, l)}^{(N)}\right)\left(\frac{\beta^{(N)}+1-e^{-\kappa \beta^{(N)}}}{\beta^{(N)}}\right)^{2} e^{2 G^{+}\left(\beta^{(N)}\right)}$.

As

$$
\lim _{N \rightarrow \infty} e^{2 G^{+}\left(\beta^{(N)}\right)}=1
$$

and

$$
\lim _{N \rightarrow \infty} \frac{\beta^{(N)}+1-e^{-\kappa \beta^{(N)}}}{\beta^{(N)}}=1+\kappa,
$$

it then follows that

$$
\lim _{N \rightarrow \infty} \frac{p_{\max }^{(N)}}{\beta^{(N)}} \leq \gamma(1+\kappa)^{2} \lim _{N \rightarrow \infty} \lambda_{\max }^{(N)}
$$

where

$$
\lambda_{\max }^{(N)}=\max _{(i, j) \in \mathcal{L}} \lambda_{(i, j)}^{(N)} .
$$

Combining the above results with the fact that for $\left\{\lambda^{(N)}\right\}_{N \geq 1} \in \mathcal{A}$ we have

$$
\limsup _{N \rightarrow \infty}\left(\max _{(i, j) \in \mathcal{L}^{(N)}} \lambda_{(i, j)}^{(N)}\right)=0,
$$

it follows that

$$
\lim _{N \rightarrow \infty} \frac{p_{m a x}^{(N)}}{\beta^{(N)}}=0 .
$$

Furthermore, using (39) we have

$$
\begin{array}{r}
\sum_{j \in \mathcal{N}_{i}^{(N)}} \frac{\left[p_{(i, j)}^{(N)}+p_{(j, i)}^{(N)}\right]}{\beta^{(N)}}=\sum_{j \in \mathcal{N}_{i}^{(N)}}\left\{\left(\bar{\lambda}_{(i, j)}^{(N)}+\bar{\lambda}_{(j, i)}^{(N)}\right) e^{2 G^{+}\left(\beta^{(N)}\right)} .\right. \\
\left.\frac{\left(\beta^{(N)}+1-e^{\left.-G_{i}^{(N)}\right)\left(\beta^{(N)}+1-e^{-G_{j}^{(N)}}\right)}\right.}{\left(\beta^{(N)}\right)^{2}}\right\} .
\end{array}
$$

Using (37), (41), and (42), it then follows that there exists a integer $N_{1}$ such that for $N \geq N_{1}$ we have

$$
\begin{aligned}
\sum_{j \in \mathcal{N}_{i}^{(N)}} \frac{\left[p_{(i, j)}^{(N)}+p_{(j, i)}^{(N)}\right]}{\beta^{(N)}} & \leq 2(1+\kappa)^{2} \sum_{j \in \mathcal{N}_{i}^{(N)}}\left[\bar{\lambda}_{(i, j)}^{(N)}+\bar{\lambda}_{(j, i)}^{(N)}\right] \\
& \leq 2(1+\kappa)^{2} \bar{\Lambda}_{i}^{(N)} \\
& \leq 2(1+\kappa)^{2} \Lambda^{*}, \quad i=1, \cdots, N .
\end{aligned}
$$

Hence the sequence $\left\{\mathbf{p}^{(N)}\right\}_{N \geq N_{0}}$ satisfies Assumption 2 and the theorem follows if we can verify (40), i.e. if we can show that there exists a constant $\kappa$ such that for all $N \geq N_{0}$ and all $i \in \mathcal{N}$, we can find a $G_{i}^{(N)}, G_{i}^{(N)} \geq 0$, that satisfies the inequality

$$
G_{i}^{(N)} \leq \kappa \beta^{(N)}
$$

and is a solution to (38), i.e. for $\beta=\beta^{(N)}$ we have that

$$
e^{\left(G_{i}^{(N)}-G^{+}(\beta)\right)} \tau\left(G_{i}^{(N)}\right) e^{-G^{+}(\beta)}=\bar{\Lambda}_{i}^{(N)}
$$

where

$$
\tau(G)=\frac{G e^{-G}}{\beta+1-e^{-G}} .
$$

Note that the function

$$
f(G)=e^{\left(G-G^{+}(\beta)\right)} \tau(G) e^{-G^{+}(\beta)}
$$

is continuous in $G$ with $f(0)=0$, and recall that by definition

there exist a positive constant $\bar{\Lambda}$ and a integer $\bar{N}$ such that for all $N \geq \bar{N}$ we have that

$$
\Lambda_{i}^{(N)} \leq \bar{\Lambda}<1, \quad i=1, \ldots, N .
$$

Therefore, in order to verify (40) it suffices to show that there exists a constant $\kappa$ such that

$$
G_{\max }^{(N)}=\kappa \beta^{(N)}
$$


we have

$$
\lim _{N \rightarrow \infty} e^{\left(G_{\max }^{(N)}-G^{+}\left(\beta^{(N)}\right)\right)} \tau\left(G_{\max }^{(N)}\right) e^{-G^{+}\left(\beta^{(N)}\right)}>\bar{\Lambda} .
$$

Using the definition of $\tau\left(G_{\max }^{(N)}\right)$, this is equivalent to showing that

$$
\begin{array}{r}
\lim _{N \rightarrow \infty} e^{\left(G_{\max }^{(N)}-G^{+}\left(\beta^{(N)}\right)\right)} \frac{G_{\max }^{(N)} e^{-G_{\max }^{(N)}}}{\beta^{(N)}+1-e^{-G_{\max }^{(N)}}} e^{-G^{+}\left(\beta^{(N)}\right)} \\
=\lim _{N \rightarrow \infty} \frac{G_{\max }^{(N)}}{\beta^{(N)}+1-e^{-G_{\max }^{(N)}}} e^{-2 G^{+}\left(\beta^{(N)}\right)}>\bar{\Lambda} .
\end{array}
$$

Recall that

$$
\lim _{N \rightarrow \infty} \frac{\kappa \beta^{(N)}}{\beta^{(N)}+1-e^{-\kappa \beta^{(N)}}}=\frac{\kappa}{1+\kappa}
$$

and $\bar{\Lambda}<1$. Combining the above results, it follows that for

$$
\kappa>\frac{\bar{\Lambda}}{1-\bar{\Lambda}}
$$

and $G_{\max }^{(N)}=\kappa \beta^{(N)}$, we have

$$
\begin{array}{r}
\lim _{N \rightarrow \infty} e^{\left(G_{\max }^{(N)}-G^{+}\left(\beta^{(N)}\right)\right)} \frac{G_{\max }^{(N)} e^{-G_{\max }^{(N)}}}{\beta^{(N)}+1-e^{-G_{\max }^{(N)}}} e^{-G^{+}\left(\beta^{(N)}\right)} \\
=\frac{\kappa}{1+\kappa}>\bar{\Lambda} .
\end{array}
$$

This verifies (40) and completes the proof.

Peter Marbach (M '01) was born in Lucerne, Switzerland. He received the Eidg. Dipl. El.-Ing. (1993) from the ETH Zurich, Switzerland, the M.S. (1994) in electrical engineering from the Columbia University, NY, U.S.A, and the Ph.D. (1998) in electrical engineering from the Massachusetts Institute of Technology (MIT), Cambridge, Massachusetts, U.S.A. He was appointed as assistant professor in 2000, and associate professor in 2005, at the Department of Computer Science of the University of Toronto. He has also been a visiting professor at Microsoft Research, Cambridge, UK, at the Ecole Polytechnique Federal at Lausanne (EPFL), Switzerland, and at the Ecole Normale Superieure, Paris, France, and a post-doctoral fellow at Cambridge University, UK

Peter Marbach has received the IEEE INFOCOM 2002 Best Paper Award for his paper "Priority Service and Max-Min Fairness". He was on the editorial board of the ACM/IEEE Transactions of Networking. His research interests are in the fields of communication networks, in particular in the area of wireless networks, peer-to-peer networks, and online social networks.

Atilla Eryilmaz (S '00-M '06) received his B.S. degree in Electrical and Electronics Engineering from Bogazaziçi University, Istanbul, in 1999, and the M.S. and Ph.D. degrees in Electrical and Computer Engineering from the University of Illinois at Urbana-Champaign in 2001 and 2005, respectively. Between 2005 and 2007, he worked as a Postdoctoral Associate at the Laboratory for Information and Decision Systems at the Massachusetts Institute of Technology.

Since 2007, he is an Assistant Professor of Electrical and Computer Engineering at The Ohio State University. He has received the NSF Career Award and the Lumley Research Award for Outstanding Research Accomplishments in 2010. His research interests include: design and analysis for communication networks, optimal control of stochastic networks, optimization theory, distributed algorithms, stochastic processes, and information theory.
Asu Ozdaglar (M '95) received the B.S. degree in Electrical Engineering from the Middle East Technical University, Ankara, Turkey, in 1996, and the S.M. and the Ph.D. degrees in Electrical Engineering and Computer Science from the Massachusetts Institute of Technology, Cambridge, in 1998 and 2003, respectively.

Since 2003, she has been a member of the faculty of the Electrical Engineering and Computer Science Department at the Massachusetts Institute of Technology, where she is currently the Class of 1943 Associate Professor. She is affiliated with the Laboratory for Information and Decision Systems and the Operations Research Center at MIT. Her research interests include optimization theory, with emphasis on nonlinear programming and convex analysis, game theory, with applications in communication, social, and economic networks, and distributed optimization and control. She is the co-author of the book entitled "Convex Analysis and Optimization". She is the recipient of a Microsoft fellowship, the MIT Graduate Student Council Teaching award, the NSF Career award, and the 2008 Donald P. Eckman award of the American Automatic Control Council. She is a member of the Board of Governors of the Control System Society. 